\title{
Modelos de Apreçamento com Influência Social
}

\author{
Rogério de Assis Medeiros
}

TESE APRESENTADA

$\mathrm{AO}$

INSTITUTO DE MATEMÁTICA E ESTATÍSTICA

DA

UNIVERSIDADE DE SÃO PAULO

PARA

OBTENÇÃO DO TÍTULO

$\mathrm{DE}$

DOUTOR EM CIÊNCIAS

Programa: Matemática Aplicada

Orientador: Prof. Dr. Renato Vicente

Durante o desenvolvimento deste trabalho o autor recebeu auxílio financeiro da CAPES

São Paulo, 19 de maio de 2017 


\section{Modelos de Apreçamento com Influência Social}

Esta tese contém as correções e alterações sugeridas pela Comissão Julgadora durante a defesa realizada por Rogério de Assis Medeiros em 19/05/2017.

O original encontra-se disponível no Instituto de Matemática e Estatística da Universidade de São Paulo.

Comissão Julgadora:

- Prof. Dr. Renato Vicente (orientador) - IME-USP

- Prof. Dr. José de Oliveira Siqueira - IP-USP

- Prof. Dr. Henrique von Dreifus - IME-USP

- Prof. Dr. André Cavalcanti Rocha Martins - EACH-USP

- Prof. Dr. Fernando Fagundes Ferreira - EACH-USP 


\section{Agradecimentos}

Gostaria de agradecer a minha família, ao meu orientador Prof. Dr. Renato Vicente (IME-USP), aos professores e funcionários do IME, IF, POLI e IMPA, aos professores que ministraram disciplinas no doutorado: Carlos Alberto de Bragança Pereira (IME-USP), Oswaldo Luiz do Valle Costa (POLI-USP), Vladimir Belitsky (IME-USP), Edson de Faria (IME-USP), Artur Lopes (UFRGS), Oscar João Abdonour (IME-USP), Nestor Caticha (IF-USP) e Adílson Simonis (IME-USP), aos professores da minha banca de qualificação: Henrique von Dreifus (IME-USP) e José de Oliveira Siqueira (IP-USP), aos colegas e/ou professores que muito contribuíram com conversas, sugestões, críticas e comentários, em especial ao professor Jairo Zacarias Gonçalves (IME-USP), e também aos professores membros da minha banca examinadora. Um agradecimento especial também ao professor Pedro Paulo Serpa Schirmer, cujo curso, Teoria Matemática de Apreçamento de Ativos (MAP5736) no verão de 2007, inspirou muito deste trabalho. Além disso, partes dos capítulos 3 e 4 foram apresentados como pôster no congresso "Research in Options 2016" no IMPA e gostaria de agradecer pelas sugestões e comentários apresentados por Bruno Dupire (Bloomberg), Chris Rogers (Cambridge University) e Max Souza (UFF). 


\section{Dedicatória:}

Dedico este trabalho aos meus pais, Sebastião Joaquim de Medeiros e Naide de Assis Medeiros, que muito lutaram para que fosse possível que eu conseguisse chegar aonde estou agora e poder prosseguir em minha trajetória. 


\section{Resumo}

\section{Modelos de Apreçamento com Influência Social}

Nesta tese desenvolvemos modelos de apreçamento de ativos financeiros baseados no conceito de influência social, analisamos também algumas das consequências destes modelos e comparamos com os modelos correspondentes clássicos.

Por meio das funções de utilidade generalizadas exponencial e quadrática, deduzimos o CAPM com influência social. Obtivemos que o coeficiente $\beta_{i}$ da fórmula do CAPM depende de uma aversão ao risco efetiva do mercado que depende da distribuição de riqueza dos agentes do mercado. Supondo que distribuição de riqueza dos agentes do mercado segue uma distribuição de Pareto, fomos capazes de conectar, aversão ao risco média efetiva do mercado, volatilidade e distribuição de riqueza dos agentes, estabelecendo a previsão empírica de que a volatilidade aumenta com a concentração da distribuição de riqueza dos agentes do mercado, a qual foi corroborada por meio de análise estatística.

Através da função generalizada tipo potência são feitas algumas considerações sobre alguns "puzzles" econômicos bem conhecidos (o "Equity Premium Puzzle"e o "Riskfree Rate Puzzle") que mostram que a modelagem da influência social pode ter impacto no esclarecimento destes "puzzles".

Palavras-chave: Finanças Comportamentais, Influência Social, CAPM, Funções de Utilidade, Aversão ao Risco 


\section{Abstract \\ Pricing Models with Social Influence}

In this thesis we develop pricing models for financial assets based in the concept of social influence, we analyze too some of consequences of this models and we compare with the corresponding classical models.

By means of the exponential and quadratic generalized utility functions, we deduce the CAPM with social influence. We obtained that the coefficient $\beta_{i}$ from the formula of the CAPM depends of a market effective risk aversion that depends of the wealth distribution of the market agents. Supposing that the wealth distribution of the market agents follows a Pareto distribution, we were able to connect, market effective average risk aversion, volatility and wealth distribution of the agents, establishing the empirical forecasting that the volatility grows with the concentration of the wealth distribution of the market agents, which was corroborated by means of statistical analysis.

Through the generalized power function are made some considerations about some economic puzzles well-known (the Equity Premium Puzzle and the Riskfree Rate Puzzle) that show us that the modeling of the social influence can to have impact in the clarification these puzzles.

Keywords: Behavioral Finance, Social Influence, CAPM, Utility Functions, Risk Aversion 


\section{Sumário}

1 Introdução 10

2 Funções de Utilidade, CAPM e "Puzzles"

Econômicos $\quad 13$

3 Influência Social $\quad 16$

3.1 Introdução . . . . . . . . . . . . . . . . . . . . . . 16

3.2 Funções de Utilidade . . . . . . . . . . . . . . . . . . . . . . . 18

3.2.1 Utilidade Exponencial . . . . . . . . . . . . . . . . . 19

3.2 .2 Utilidade Quadrática . . . . . . . . . . . . . . . . 21

3.2.3 Utilidade Potência . . . . . . . . . . . . . . . . 23

3.2.4 Utilidade Logarítmica . . . . . . . . . . . . . . 26

4 Modelo CAPM Uniperiódico com Influência Social - Utilidade Exponencial $\quad 29$

4.1 Modelo do Mercado . . . . . . . . . . . . . . . . . . . . . . 29

4.2 CAPM com Influência Social . . . . . . . . . . . . . . . . 40

4.3 Analisando Algumas Consequências . . . . . . . . . . . . . . 44

5 Modelo CAPM Uniperiódico com Influência Social - Utili$\begin{array}{ll}\text { dade Quadrática } & 49\end{array}$

5.1 Modelo do Mercado . . . . . . . . . . . . . . . . . . . . . . 49

5.2 CAPM com Influência Social . . . . . . . . . . . . . . . . 61

5.3 Analisando Algumas Consequências . . . . . . . . . . . . 66

6 Evidências Empíricas $\quad 72$

6.1 Índices . . . . . . . . . . . . . . . . . . . . . . . 72

6.2 Apresentação dos Dados e ANOVA . . . . . . . . . . . . 73

7 Considerações sobre "Puzzles" Econômicos 78

7.1 Os Puzzles . . . . . . . . . . . . . . . . . . . 78

7.2 Função de Utilidade do Consumo . . . . . . . . . . . . . . . . 80

7.3 Modelando o Fator de Desconto Estocástico . . . . . . . . . . 82

7.4 Aplicando a Utilidade Generalizada no Fator de Desconto Estocástico . . . . . . . . . . . . . . . . 84

7.5 Considerações sobre os Puzzles . . . . . . . . . . . . . . . 88

8 Conclusão e Perspectivas $\quad 92$

$\begin{array}{ll}\text { Referências } & 94\end{array}$ 


\section{Lista de Figuras}

1 Aversão ao risco média efetiva $\left(\theta_{m}\right)$ versus índice de Gini $(\mathrm{G})$ - Utilidade Exponencial . . . . . . . . . . . . . . . 47

2 Aversão ao risco média efetiva $\left(\theta_{m}\right)$ versus índice de Gini $(\mathrm{G})$ $\left(\lambda_{1}=10^{5}\right)$ - Utilidade Quadrática . . . . . . . . . . 70

3 Aversão ao risco média efetiva $\left(\theta_{m}\right)$ versus índice de Gini $(\mathrm{G})$ $\left(\lambda_{1}=10^{6}\right)$ - Utilidade Quadrática . . . . . . . . . 70

4 Volatilidade $(\sigma)$ versus índice Herfindahl-Hirschman normalizado $\left(H H I m^{*}\right) \ldots \ldots \ldots \ldots$. . . . . . . . . . 76

$5 \quad$ Volatilidade $(\sigma)$ versus índice Herfindahl-Hirschman normalizado $\left(H H I a^{*}\right) \ldots \ldots \ldots \ldots$. . . . . . . . . . . . . . 77 


\section{Introdução}

Personally, I don't see old economics and behavioural economics as opposed. It is useful to assume people are rational as a good approximation to their long term behaviour, but it would be unwise not to think how in practice their behaviour may deviate from that simplifying assumption.

Evan Davis

Nesta tese, o objetivo principal é estabelecer um novo "framework" que incorpora a presença de influência social de agentes econômicos (em particular agentes financeiros) sobre a tomada de decisão de um dado agente $a$.

Na teoria econômica tradicional, (como pode ser vista em [mas-colell][18] ou [kreps][16]) os agentes econômicos agem segundo a racionalidade econômica (representados através de funções de utilidade), e podem ser vistos como gananciosos ("quanto mais melhor"). Aqui, os agentes econômicos continuarão a ser gananciosos, mas serão também invejosos ("querem ter mais do que os demais").

Partimos da teoria tradicional, baseada em funções de utilidade e acrescentamos um termo perturbativo que captura a presença da influência social. Este termo perturbativo tem alguns ingredientes que serão detalhados nos próximos capítulos, entre eles, uma estrutura probabilística heterogênea que representa as expectativas do agente $a$ sobre as ações dos demais. Esta estrutura pode ser interpretada como um "véu".

O uso de modelos perturbativos é algo usual em Mecânica Celeste, onde a interação gravitacional entre um planeta e o Sol é afetada pela presença dos demais corpos celestes (pense por exemplo, no caso do Sistema Solar); esta presença "perturba" a interação principal, criando desvios no movimento dos corpos, e seu estudo é feito com a inclusão de termos de ordem superior (no sentido da Série de Taylor). Este tipo de técnica é usual também em outras áreas da Física como em Mecânica Quântica, Física do Estado Sólido, Teoria Quântica de Campos, ... O tratamento perturbativo nestes modelos serviu de inspiração para nossa abordagem da influência social. Veja [butkov][6], capítulo 15, para observar como o uso de modelos perturbativos é feito em Física. 
Observe que estamos agindo de acordo com a citação que abre esta Introdução, ou seja, nosso "approach" pode ser visto como uma correção do tradicional, buscando tornar a teoria mais próxima da realidade.

No capítulo 2 relatamos de modo mais detalhado e histórico os conceitos de função de utilidade, e consideramos os tópicos a serem tratados por meio de influência social: o modelo CAPM (Capital Asset Pricing Model) e os "puzzles" econômicos "equity premium puzzle" (EPP) e "riskfree rate puzzle"(RRP).

No capítulo 3 apresentamos os conceitos de função de utilidade generalizada e função de utilidade efetiva, ambos baseados na influência social. São também definidas e discutidas as propriedades das funções de utilidade a serem utilizadas nos próximos capítulos, a saber, a exponencial, a quadrática, a potência e a logarítmica.

No capítulo 4, de posse da função generalizada exponencial, e fazendo hipóteses sobre o comportamento das crenças de um agente $a$ sobre os demais (representado por uma distribuição multivariada normal) deduzimos o CAPM com influência social. Entre os principais resultados temos que o coeficiente $\beta_{i}$ da fórmula do CAPM depende de uma aversão ao risco efetiva do mercado que por sua vez, depende da distribuição de riqueza dos agentes do mercado. Supondo que distribuição de riqueza dos agentes do mercado segue uma distribuição de Pareto com índice de Gini $G$ fomos capazes de conectar, aversão ao risco média efetiva do mercado, volatilidade e distribuição de riqueza dos agentes, estabelecendo a previsão empírica de que a volatilidade aumenta com a concentração da distribuição de riqueza dos agentes do mercado (note que este resultado é algo presente somente em nosso modelo, com influência social). Um resultado a ser notado é que a aversão ao risco média efetiva do mercado é obtida de maneira explícita em função do índice de Gini do mercado.

No capítulo seguinte (o quinto), agora com a função generalizada quadrática, retomamos os passos do capítulo anterior, porém relaxando as hipóteses sobre o comportamento das crenças de um agente $a$ sobre os demais (somente fazendo considerações a respeito dos dois primeiros momentos) e novamente deduzimos o CAPM com influência social. Os resultados do capítulo anterior são novamente recuperados, porém notamos que a aversão ao risco média efetiva do mercado, obtida de maneira explícita em função do índice de Gini do mercado tem outra expressão. A previsão empírica de a volatilidade aumenta com a concentração da distribuição de riqueza dos agentes do mercado se manteve. 
No capítulo sexto, apresentamos evidências empíricas que corroboram nossa previsão de que a volatilidade aumenta com a concentração da distribuição de riqueza dos agentes do mercado, isto é feito utilizando como "proxy" para o índice de Gini da distribuição de riqueza dos agentes do mercado, o índice Herfindahl-Hirschman normalizado $\left(H H I^{*}\right)$.

No capítulo seguinte (o sétimo), através da função generalizada tipo potência são feitas algumas considerações sobre alguns "puzzles" econômicos bem conhecidos (o "Equity Premium Puzzle"e o "Riskfree Rate Puzzle") que mostram que a modelagem da influência social pode ter impacto no esclarecimento destes "puzzles".

Finalmente, no último capítulo discutimos algumas perspectivas futuras de trabalho, tendo em vista o "framework" apresentado, ou seja, a inclusão da influência social, via função de utilidade, para desenvolvimento da teoria econômica e financeira. 


\title{
2 Funções de Utilidade, CAPM e "Puzzles" Econômicos
}

\begin{abstract}
Economics...examines that part of individual and social action which is most closely connected with the attainment and with the use of the material requisites of wellbeing. Thus it is on the one side a study of wealth; and, on the other, and more important side, a part of the study of man.
\end{abstract}

\section{Alfred Marshall \\ Principles of Economics}

\begin{abstract}
Adam Smith (1723-1790), em sua obra principal, "The Wealth of Nations", examinou diversas questões econômicas. Partindo de uma visão que o ser humano é um egoísta inato e que age em seu próprio benefício, Smith criou uma teoria econômica a respeito de como se dão as trocas econômicas. Smith e outros pensadores, entre eles, Malthus, David Ricardo, Stuart Mill e Say, ficaram conhecidos como economistas clássicos.
\end{abstract}

A partir de 1870, o economista austríaco Karl Menger (1840-1921) iniciou uma abordagem baseada na satisfação dada e valoração subjetiva dos bens, ou seja, na utilidade proporcionada por estes. Observando que em geral os bens são escassos em relação às necessidades humanas e com base em escalas de preferências de um indivíduos em relação a vários bens, Menger e seus seguidores (chamados de neoclássicos ou marginalistas) procuraram reconstruir a teoria econômica. Indo além dos clássicos, fundamentaram suas ideias numa teoria de valor baseada na utilidade subjetiva proporcionada por um bem que existe em quantidade limitada (surgindo daí a análise na margem).

Surge assim o "homo economicus", um sujeito que age economicamente procurando maximizar sua utilidade, dadas restrições de escassez; com o tempo esta noção passa a ser a de racionalidade econômica. A utilidade de um bem devia satisfazer duas condições: (i) quanto mais melhor, ou seja, um aumento na quantidade do bem, leva a um aumento de utilidade dada por este (utilidade crescente) e (ii) a satisfação proporcionada por um acréscimo de um bem é cada vez menor (utilidade marginal decrescente). Para mais detalhes históricos veja [pinho][22]. 
No século XX, John von Neumann (1913-1957) e Oskar Morgenstern (19021977), na segunda edição da obra "Theory of Games and Economic Behavior" de 1947, utilizaram o princípio da utilidade esperada, que permitiu tratar de situações com incerteza. Assim, em cenários que envolvem o acaso, a escolha é baseada em qual fornece o maior valor esperado da função de utilidade (note que agora, o tratamento passa a ser eminentemente matemático da utilidade, deixando de lado, muito da discussão filosófica gerada nos anos iniciais do conceito).

Na década seguinte, Gerard Debreu (1921-2004) na obra "Theory of Value" de 1959, seguindo influência do grupo francês de matemáticos Bourbaki, estabelece a teoria econômica de maneira axiomática e plenamente matemática, indo muito além dos trabalhos de Walras. Partindo da noção de preferência (representada como uma ordenação no espaço de bens) e a seguir estabelecendo condições para existência da função de utilidade, constrói-se uma teoria axiomática do consumidor e desenvolve-se a Microeconomia. Esta é a abordagem consagrada no ensino até hoje, como podemos ver entre alguns dos principais livros de Microeconomia (por exemplo, [mas-colell][18] e $[$ kreps $][16])$.

Ainda na década de 50, estas ideias começam a ser aplicadas no campo das Finanças, Harry Markowitz desenvolve sua Teoria de Carteiras, baseado num tratamento matemático do comportamento do investidor em que este, preocupado com o retorno e o risco de sua carteira, procurar maximizar a utilidade esperada de sua riqueza (um problema de maximização quadrática).

Nos anos 60, com os trabalhos de Sharpe, Lintner e Mossin é estabelecido um modelo de formação de preços para ativos com risco (CAPM ou Capital Asset Pricing Model). No CAPM, o excesso de retorno de cada ativo com risco está linearmente relacionado com um índice que reflete o comportamento do mercado como um todo (na clássica expressão $r_{i}-r_{f}=\beta_{i}\left(r_{M}-r_{f}\right)$ ). Para mais detalhes históricos veja [araujo][2].

Em 1985, Rajnish Mehra e Edward C. Prescott publicaram o trabalho "The Equity Premium: A Puzzle", onde identificaram que o prêmio de risco (excesso de retorno) requerido para as ações em relação aos títulos públicos para o mercado americano era alto demais. Neste artigo, partindo de um modelo onde os agentes têm uma função de utilidade do consumo separável no tempo e há somente um parâmetro livre, o coeficiente de aversão ao risco; foram capazes de relacionar de maneira quantitativa o prêmio de risco exigido com o coeficiente de aversão ao risco. Abreviadamente este "puzzle"é conhecido 
como EPP.

O parâmetro obtido no modelo deveria estar entre 30 e 40, considerando os dados mensuráveis, sendo que as estimativas apontam para algo entre 1 e 2 (veja [thaler][30]). Diversas abordagens foram tentadas desde então para explicar o "puzzle", que se enquadram nas seguintes categorias: (i) procurar investigar melhor os dados de modo a verificar as evidências empíricas; (ii) criar novas estruturas teóricas que possam modelar de maneira mais adequada os fenômenos observados; (iii) negar o "puzzle" procurando justificar um coeficiente de aversão ao risco elevado (veja [thaler][30] e [samanez][23]). Nossa abordagem nesta tese segue a opinião (ii). Note que a presença deste "puzzle" foi verificada em outros países, mas não em todos, em particular há muita controvérsia a respeito de sua presença no mercado brasileiro (veja [samanez][23] e [guimaraes][12]).

No decorrer das investigações do EPP, foi encontrada a presença de outro "puzzle": Philippe Weil no artigo "The Equity Premium Puzzle and the Riskfree Rate Puzzle" de 1989, observou que pelo mesmo modelo que leva ao EPP, as taxas de juros dos ativos livres de risco (títulos públicos) são baixas demais, este é o "Riskfree Rate Puzzle" (abreviado por RRP).

Críticas a abordagem baseada numa noção simplificada do comportamento humano foram feitas desde o início do Marginalismo, como podemos observar na citação de Marshall que abre o capítulo, além dele, outros economistas também procuraram ressaltar as limitações da racionalidade econômica e suas consequências, entre eles, Pigou, Veblen e Keynes. Com os trabalhos de Daniel Kahneman e Amos Tversky nos anos 70, iniciou-se a chamada Economia Comportamental que faz uma crítica aos pressupostos do comportamento do "homo economicus". Por sua Teoria da Perspectiva ("Prospect Theory"), Kahneman recebeu o prêmio Nobel de Economia de 2002.

No próximo capítulo vamos abordar as noções aqui apresentadas com o uso de um novo ingrediente: a influência social. Com ela pretendemos mostrar que a visão tradicional pode ser aprimorada de modo a se aproximar do comportamento humano real. 


\section{Influência Social}

Investing in speculative assets is a social activity. Investors spend a substantial part of their leisure time discussing investments, reading about investments, or gossiping about others' sucesses or failures in investing.

Robert J. Shiller

Stock Prices and Social Dynamics

\subsection{Introdução}

Os modelos tradicionais em Finanças Matemáticas assumem pressupostos sobre o comportamento dos investidores, que são baseados na noção de racionalidade econômica (a mesma utilizada de modo geral para construir toda a Microeconomia). Tais modelos fazem hipóteses sobre o comportamento dos investidores que são encapsulados na função de utilidade, à qual associa a um dado nível de riqueza esperada, um certo nível de satisfação. A função de utilidade deve ter sua primeira derivada positiva (quanto mais melhor) e a segunda negativa (a satisfação proporcionada pelos incrementos é cada vez menor). Note que esta modelagem é baseada apenas numa visão de que o comportamento individual intrínseco não sofre influência dos demais agentes do mercado, assim a interação entre os participantes do mercado se dá apenas pelo mecanismo de preços.

Do ponto de vista do real comportamento dos investidores são observadas interações sociais entre estes, o que leva a possibilidade de existir influência social. Mas o que significa, para nós, influência social?

Aqui influência social significa que o comportamento de um investidor é influenciado pelas crenças que este tem sobre o comportamento dos demais investidores. Como seu comportamento é determinado pela sua função de utilidade, o que temos é que esta função depende também destas crenças (expectativas), em outras palavras, a satisfação de um agente é influenciada pelas crenças que ele tem nas ações dos demais agentes.

Como podemos observar existem dois ingredientes na influência social: (i) uma função de utilidade que depende das crenças de um agente sobre as 
ações dos demais e (ii) um canal de comunicação que permita a formação das crenças do agente sobre os demais. Assim, como observado na citação de abertura deste capítulo, os agentes do mercado se comunicam entre si o tempo todo, por meio de conversas, recomendações, via imprensa, internet, ... o que garante a presença do canal de comunicação para formação das expectativas. Note que a formação das crenças pode ter influência de agentes (não necessariamente pessoas físicas) de fora do mercado, como governo, imprensa, ...

Olhando de um ponto de vista comportamental (psicológico) podemos caracterizar a abordagem tradicional como baseada apenas na "ganância" do investidor (a primeira derivada positiva da função de utilidade é sempre positiva), mas a realidade nos leva a tentar capturar também, outro sentimento ou viés cognitivo: a "inveja", ou seja, um investidor não quer apenas ser mais rico (ganância) mas também ser mais rico que os outros, em particular do que aqueles que estão em sua rede social.

O comportamento econômico de um agente continua sendo guiado pela maximização de sua utilidade (satisfação), como ele prefere sempre mais de um bem do que menos, dizemos que nossos agentes são gananciosos (note que este comportamento só depende do próprio agente, por isso dizemos que ganância é individual). Em nossa modelagem, os agentes econômicos também estão interessados em ter mais do que os demais, por isso dizemos que eles são também invejosos (note que ter mais que outros é algo que depende dos demais agentes, por isso dizemos que inveja é social).

Neste texto estamos interessados em capturar ambos: ganância e inveja, porém isto é feito dando um grau maior de importância para a ganância e numa escala menor a inveja, assim, vamos fazê-lo de modo perturbativo em nosso modelo matemático. Se por um lado ganância é algo individual, por outro, inveja é algo necessariamente social, ou seja, depende do comportamento dos outros investidores, o qual não é exatamente conhecido e assim somos levados ao outro ingrediente de nossa abordagem: uma estrutura probabilística de crenças heterogêneas das ações tomadas pelos outros agentes do mercado, a qual depende de cada agente e de cada ativo. Esta estrutura funciona como um "véu" que cobre as ações dos demais agentes e impede que um dado agente $a$ conheça exatamente as ações dos demais. Este "véu" encapsula toda a informação que o agente $a$ tem ou acredita sobre os demais.

A princípio podemos ter interação social entre todos os agentes do mercado, caso a rede de influência social seja mais restrita, teremos algumas 
correlações nulas em nosso modelo, na estrutura probabilística de crenças das ações tomadas pelos outros agentes do mercado. A estrutura probabilística de retornos dos ativos continua sendo homogênea e conhecida de todos agentes do mercado.

Em sua obra "The General Theory of Employment, Interest and Money" de 1936, Keynes descreve a seguinte situação (conhecida como concurso de beleza de Keynes): suponha um concurso fictício onde os participantes, devem escolher as mais belas garotas dentre um certo conjunto de fotos conhecidas por todos os participantes, os ganhadores do concurso serão aqueles que acertarem quais são as mais belas escolhidas. A princípio, uma estratégia para um participante seria escolher aquelas que ele considera mais bonitas, porém o que realmente importa é acertar as ganhadoras, logo ele deveria procurar escolher aquelas que seriam mais escolhidas pelos demais. Keynes coloca este exemplo como representando o comportamento observado no mercado financeiro, onde os investidores buscam os ativos que estarão mais valorizados (por serem mais procurados pelos demais). Nosso tratamento segue numa linha muito próxima a sugerida por Keynes.

Entre os estudos sobre influência social em mercados financeiros, (entre eles [anderson][1], [ozsoylev][19] e [scheinkman2008][25]) a modelagem é em geral muito diferente da nossa abordagem, em [scheinkman2006][24] é apresentada algo semelhante a utilidade generalizada desta tese, com a utilidade de um agente $a$ dependendo do valor esperado das ações dos demais agentes vizinhos, porém o desenvolvimento segue uma linha diferente da nossa. Existem alguns estudos que constroem modelos de CAPM comportamental, mas eles não usam a influência social diretamente ([paudel][21], [shefrin1994][27], [shefrin2000][28]).

Dupire ([dupire][10]) utilizou uma função de utilidade generalizada para modelar outro "sentimento" ou viés cognitivo, o arrependimento ("regret"), por meio de um "toy model".

\subsection{Funções de Utilidade}

O comportamento individual dos investidores é encapsulado matematicamente por meio da função de utilidade $U(V)$, a qual, dado um valor financeiro $V$ (riqueza, dotação orçamentária, ...) fornece um nível de satisfação. Esta função deve ser pelo menos duas vezes diferenciável e deve ser crescente 
$\left(U^{\prime}(V)>0\right)$ e também côncava $\left(U^{\prime \prime}(V)<0\right)$, ou seja, com utilidade marginal decrescente $\left(U^{\prime}(V)\right.$ é a utilidade marginal).

Neste trabalho estamos utilizando o conceito de função de utilidade cardinal e tomando como propriedades definidoras apenas suas duas primeiras derivadas. Algumas abordagens fazem exigências sobre as demais derivadas (veja [billio][4]).

Algumas das principais funções de utilidade utilizadas são a exponencial, quadrática, potência e logarítmica, as quais apresentam propriedades diversas, em termos de aversão ao risco. Em nossa modelagem, a influência social é capturada na função utilidade através de termos perturbativos em que cada agente tem seu nível de satisfação influenciado pelos demais agentes do mercado.

Assim temos funções de utilidades generalizadas $\left(U\left(V_{a}, V_{-a}\right)\right)$, onde $V_{a}$ é o valor financeiro do agente $a$ e $V_{-a}$ representa os valores financeiros dos demais agentes. Como as ações dos demais agentes não são conhecidas, o agente $a$ tem um conjunto de crenças heterogêneas sobre as ações dos demais. Tal conjunto de crenças é representado por uma distribuição de probabilidades multidimensional $\pi_{a}$.

O valor esperado $\mathbb{E}_{\pi_{a}}\left[U\left(V_{a}, V_{-a}\right)\right]$ corresponde a uma utilidade generalizada efetiva, ou simplesmente utilidade efetiva. Esta utilidade efetiva é que irá fazer o papel da utilidade tradicional $U\left(V_{a}\right)$. Assim, o agente $a$ tem um nível de satisfação "percebido" (efetivo) que é influenciado pela sua percepção dos níveis de satisfação dos demais agentes.

Note que as crenças heterogêneas sobre as ações dos demais agentes agem como um "véu" que não permite ver claramente as ações dos demais agentes, assim o que cada agente $a$ tem com função de utilidade efetiva (que controla o seu comportamento) é o valor esperado na distribuição das crenças ("integral ao longo do véu").

A seguir apresentamos as funções de utilidade generalizadas a serem empregadas e o cálculo de suas principais propriedades:

\subsubsection{Utilidade Exponencial}

A função de utilidade exponencial tradicional apresenta a propriedade de reagir a variações absolutas do valor $V_{a}$ de maneira constante (independe 
de $V_{a}$ ), de modo técnico, isto significa que sua aversão ao risco absoluta é constante, vamos observar que a função de utilidade exponencial generalizada também satisfaz esta condição. A função de utilidade exponencial generalizada $\left(U\left(V_{a}, V_{-a}\right)\right)$ é dada por:

$$
\begin{aligned}
U\left(V_{a}, V_{-a}\right) & =-\exp \left(-\theta_{a} V_{a}-\frac{\lambda}{m-1} \sum_{j=1 j \neq a}^{m}\left(V_{a}-V_{j}\right)\right) \\
& =-\exp \left(-\theta_{a} V_{a}-\lambda\left(V_{a}-\bar{V}_{-a}\right)\right) \\
& =-\exp \left(-\left(\theta_{a}+\lambda\right) V_{a}+\lambda \bar{V}_{-a}\right) \\
& =-\exp \left(-\left(\theta_{a}+\lambda\right) V_{a}\right) \exp \left(\lambda \bar{V}_{-a}\right)
\end{aligned}
$$

Onde

$$
\bar{V}_{-a}=\frac{1}{m-1} \sum_{j=1 j \neq a}^{m} V_{j}
$$

ou seja, $\bar{V}_{-a}$ é a média aritmética dos valores $V_{j}$ dos demais agentes, diferentes de $a$. Os termos $\theta_{a}$ e $\lambda$ são ambos positivos, com $\theta_{a}>>\lambda$. Note que aqui o agente $a$ tem sua "satisfação" afetada pela média aritmética dos valores dos demais agentes.

A função de utilidade exponencial efetiva $\left(U_{e}\left(V_{a}\right)\right)$ é dada por:

$$
\begin{aligned}
U_{e}\left(V_{a}\right) & =\mathbb{E}_{\pi_{a}}\left[U\left(V_{a}, V_{-a}\right)\right] \\
& =-\exp \left(-\left(\theta_{a}+\lambda\right) V_{a}\right) \mathbb{E}_{\pi_{a}}\left[\exp \left(\lambda \bar{V}_{-a}\right)\right]
\end{aligned}
$$

Vamos calcular a primeira e a segunda derivadas da utilidade efetiva para verificar que satisfaz as condições de uma função de utilidade:

$$
\frac{d U_{e}\left(V_{a}\right)}{d V_{a}}=\left(\theta_{a}+\lambda\right) \exp \left(-\left(\theta_{a}+\lambda\right) V_{a}\right) \mathbb{E}_{\pi_{a}}\left[\exp \left(\lambda \bar{V}_{-a}\right)\right]>0
$$

Note que $\mathbb{E}_{\pi_{a}}\left[\exp \left(\lambda \bar{V}_{-a}\right)\right]$ é positivo, pois se trata da esperança de uma 
variável aleatória positiva (exponencial).

$$
\begin{aligned}
\frac{d^{2} U_{e}\left(V_{a}\right)}{d V_{a}^{2}} & =-\left(\theta_{a}+\lambda\right)^{2} \exp \left(-\left(\theta_{a}+\lambda\right) V_{a}\right) \mathbb{E}_{\pi_{a}}\left[\exp \left(\lambda \bar{V}_{-a}\right)\right] \\
& =-\left(\theta_{a}+\lambda\right) \frac{d U_{e}\left(V_{a}\right)}{d V_{a}}<0
\end{aligned}
$$

O coeficiente de aversão ao risco absoluto (Arrow-Pratt) é dado por:

$$
A R_{a}\left(U_{e}\right)=-\frac{U_{e}^{\prime \prime}\left(V_{a}\right)}{U_{e}^{\prime}\left(V_{a}\right)}=\theta_{a}+\lambda
$$

O coeficiente de aversão ao risco relativo (Arrow-Pratt) é dado por:

$$
A R_{r}\left(U_{e}\right)=V_{a} \cdot A R_{a}\left(U_{e}\right)=V_{a} \cdot\left(\theta_{a}+\lambda\right)
$$

Portanto é uma função utilidade tipo CARA ("Constant Absolute Risk Aversion"), ou seja, com aversão ao risco absoluta constante.

Nas demais seções deste capítulo vamos omitir a dependência explícita dos parâmetros das funções de utilidade em relação ao agente $a$ para simplificar a notação.

\subsubsection{Utilidade Quadrática}

A função de utilidade quadrática generalizada $\left(U\left(V_{a}, V_{-a}\right)\right)$ é dada por:

$$
\begin{aligned}
U\left(V_{a}, V_{-a}\right) & =c V_{a}+\frac{\lambda_{1}}{m-1} \sum_{j=1 j \neq a}^{m}\left(V_{a}-V_{j}\right)-\frac{b}{2}\left(V_{a}\right)^{2}-\frac{\lambda_{2}}{2(m-1)} \\
& -\sum_{j=1 j \neq a}^{m}\left(\left(V_{a}\right)^{2}-\left(V_{j}\right)^{2}\right) \\
& =c V_{a}+\lambda_{1}\left(V_{a}-\bar{V}_{-a}\right)-\frac{b}{2}\left(V_{a}\right)^{2}-\frac{\lambda_{2}}{2}\left(\left(V_{a}\right)^{2}-\overline{V_{-a}^{2}}\right) \\
& =\left(c+\lambda_{1}\right) V_{a}-\lambda_{1} \bar{V}_{-a}-\frac{\left(b+\lambda_{2}\right)}{2}\left(V_{a}\right)^{2}-\frac{\lambda_{2}}{2} \overline{V_{-a}^{2}}
\end{aligned}
$$


Onde, $\bar{V}_{-a}$ é a média aritmética dos valores $V_{j}$ dos demais agentes, diferentes de $a$ e

$$
\overline{V_{-a}^{2}}=\frac{1}{m-1} \sum_{j=1 j \neq a}^{m} V_{j}^{2}
$$

ou seja, $\overline{V_{-a}^{2}}$ é a média aritmética dos valores $V_{j}^{2}$ dos demais agentes, diferentes de $a$. Os termos $c, b, \lambda_{1}$ e $\lambda_{2}$ são todos positivos, com $c>>\lambda_{1}$ e $b>>\lambda_{2}$. Note que aqui o agente $a$ tem sua "satisfação" afetada pela média aritmética dos valores dos demais agentes. Em outras palavras, o valor (riqueza, consumo, ...) percebido de $a$ é afetado pelos dos demais.

A função de utilidade quadrática efetiva $\left(U_{e}\left(V_{a}\right)\right)$ é dada por:

$$
\begin{aligned}
U_{e}\left(V_{a}\right) & =\mathbb{E}_{\pi_{a}}\left[U\left(V_{a}, V_{-a}\right)\right] \\
& =\left(c+\lambda_{1}\right) V_{a}-\frac{\left(b+\lambda_{2}\right)}{2}\left(V_{a}\right)^{2}-\lambda_{1} \mathbb{E}_{\pi_{a}}\left[\overline{V_{-a}}\right]-\frac{\lambda_{2}}{2} \mathbb{E}_{\pi_{a}}\left[\overline{V_{-a}^{2}}\right]
\end{aligned}
$$

Vamos calcular a primeira e a segunda derivadas da utilidade efetiva para verificar que satisfaz as condições de uma função de utilidade:

$$
\begin{aligned}
\frac{d U_{e}\left(V_{a}\right)}{d V_{a}} & =\left(c+\lambda_{1}\right)-\left(b+\lambda_{2}\right) V_{a}>0 \\
& \Leftrightarrow V_{a}<\frac{c+\lambda_{1}}{b+\lambda_{2}}
\end{aligned}
$$

Este termo é positivo para valores limitados de $V_{a}$.

$$
\frac{d^{2} U_{e}\left(V_{a}\right)}{d V_{a}^{2}}=-\left(b+\lambda_{2}\right)<0
$$

O coeficiente de aversão ao risco absoluto (Arrow-Pratt) é dado por: 


$$
A R_{a}\left(U_{e}\right)=-\frac{U_{e}^{\prime \prime}\left(V_{a}\right)}{U_{e}^{\prime}\left(V_{a}\right)}=\frac{b+\lambda_{2}}{\left(c+\lambda_{1}\right)-\left(b+\lambda_{2}\right) V_{a}}
$$

O coeficiente de aversão ao risco relativo (Arrow-Pratt) é dado por:

$$
\begin{aligned}
A R_{r}\left(U_{e}\right) & =V_{a} \cdot A R_{a}\left(U_{e}\right) \\
& =V_{a} \cdot\left(\frac{b+\lambda_{2}}{\left(c+\lambda_{1}\right)-\left(b+\lambda_{2}\right) V_{a}}\right) \\
& =\frac{\left(b+\lambda_{2}\right) V_{a}}{\left(c+\lambda_{1}\right)-\left(b+\lambda_{2}\right) V_{a}}
\end{aligned}
$$

Portanto é uma função utilidade tipo HARA ("Hyperbolic Absolute Risk Aversion"), ou seja, com aversão ao risco absoluta hiperbólica. Ela tem aversão ao risco relativa crescente $\left(\frac{d A R_{r}\left(U_{e}\right)}{d V_{a}}>0\right)$.

A função de utilidade quadrática, tanto a tradicional como a generalizada tem aplicabilidade limitada, servindo como aproximação das demais (primeiros termos da Série de Taylor). Sobre a limitação da função quadrática tradicional veja $[$ costa] $[9]$.

\subsubsection{Utilidade Potência}

A função de utilidade potência tradicional apresenta a propriedade de reagir a variações relativas do valor $V_{a}$ de maneira constante (independe de $V_{a}$ ), de modo técnico, isto significa que sua aversão ao risco relativa é constante, vamos observar que função de utilidade potência generalizada também satisfaz esta condição. A função de utilidade potência generalizada $\left(U\left(V_{a}, V_{-a}\right)\right)$ é dada por: 


$$
\begin{aligned}
U\left(V_{a}, V_{-a}\right) & =\frac{V_{a}^{1-\gamma}}{1-(\gamma+\lambda)}\left(\prod_{j=1 \neq a}^{m}\left(\frac{V_{a}}{V_{j}}\right)^{\frac{1}{m-1}}\right)^{-\lambda} \\
& =\frac{V_{a}^{1-\gamma}}{1-(\gamma+\lambda)} V_{a}^{-\lambda}\left(\bar{V}_{-a}\right)^{\lambda} \\
& =\frac{V_{a}^{1-(\gamma+\lambda)}}{1-(\gamma+\lambda)}\left(\bar{V}_{-a}\right)^{\lambda}
\end{aligned}
$$

Onde

$$
\bar{V}_{-a}=\prod_{j=1 j \neq a}^{m}\left(V_{j}\right)^{\frac{1}{m-1}}
$$

ou seja, $\bar{V}_{-a}$ é a média geométrica dos valores $V_{j}$ dos demais agentes, diferentes de $a$. Os termos $\gamma$ e $\lambda$ são positivos e tais que $\gamma>\lambda, \gamma+\lambda<1$. Note que aqui o agente $a$ tem sua "satisfação" afetada pela média geométrica dos valores dos demais agentes.

Neste caso estamos supondo que todos os valores $V_{j}, 1 \leq j \leq m$ são positivos.

A função de utilidade potência efetiva $\left(U_{e}\left(V_{a}\right)\right)$ é dada por:

$$
\begin{aligned}
U_{e}\left(V_{a}\right) & =\mathbb{E}_{\pi_{a}}\left[U\left(V_{a}, V_{-a}\right)\right] \\
& =\frac{V_{a}^{1-(\gamma+\lambda)}}{1-(\gamma+\lambda)} \mathbb{E}_{\pi_{a}}\left[\left(\bar{V}_{-a}\right)^{\lambda}\right]
\end{aligned}
$$

Vamos calcular a primeira e a segunda derivadas da utilidade efetiva para verificar que satisfaz as condições de uma função de utilidade:

$$
\begin{aligned}
\frac{d U_{e}\left(V_{a}\right)}{d V_{a}} & =\frac{(1-(\gamma+\lambda)) V_{a}^{-(\gamma+\lambda)}}{1-(\gamma+\lambda)} \mathbb{E}_{\pi_{a}}\left[\left(\bar{V}_{-a}\right)^{\lambda}\right] \\
& =V_{a}^{-(\gamma+\lambda)} \mathbb{E}_{\pi_{a}}\left[\left(\bar{V}_{-a}\right)^{\lambda}\right]>0
\end{aligned}
$$


Note que $\left.\mathbb{E}_{\pi_{a}}\left[\bar{V}_{-a}\right)\right]$ é positivo, pois se trata da esperança de uma variável aleatória positiva (raiz da média geométrica de variáveis aleatórias positivas).

$$
\frac{d^{2} U_{e}\left(V_{a}\right)}{d V_{a}^{2}}=-(\gamma+\lambda) V_{a}^{-(\gamma+\lambda+1)} \mathbb{E}_{\pi_{a}}\left[\left(\bar{V}_{-a}\right)^{\lambda}\right]<0
$$

O coeficiente de aversão ao risco absoluto (Arrow-Pratt) é dado por:

$$
A R_{a}\left(U_{e}\right)=-\frac{U_{e}^{\prime \prime}\left(V_{a}\right)}{U_{e}^{\prime}\left(V_{a}\right)}=\frac{(\gamma+\lambda)}{V_{a}}
$$

O coeficiente de aversão ao risco relativo (Arrow-Pratt) é dado por:

$$
A R_{r}\left(U_{e}\right)=V_{a} \cdot A R_{a}\left(U_{e}\right)=V_{a}\left(\frac{(\gamma+\lambda)}{V_{a}}\right)=\gamma+\lambda
$$

Portanto é uma função utilidade tipo CRRA ("Constant Relative Risk Aversion"), ou seja, com aversão ao risco relativa constante.

Vamos analisar a situação em que $\gamma \longrightarrow 1-\lambda$. Temos:

$$
\begin{aligned}
\lim _{\gamma \rightarrow 1-\lambda} \frac{V_{a}^{1-(\gamma+\lambda)}-1}{1-(\gamma+\lambda)}\left(\bar{V}_{-a}\right)^{\lambda} & =\left(\bar{V}_{-a}\right)^{\lambda} \lim _{\gamma \rightarrow 1-\lambda} \frac{\exp \left((1-\lambda-\gamma) \ln V_{a}\right)-1}{(1-\lambda-\gamma)} \\
& =\left(\bar{V}_{-a}\right)^{\lambda} \ln V_{a}
\end{aligned}
$$




\subsubsection{Utilidade Logarítmica}

A função de utilidade logarítmica tradicional apresenta a propriedade de reagir a variações relativas do valor $V_{a}$ de maneira constante (independe de $V_{a}$ ), de modo técnico, isto significa que sua aversão ao risco relativa é constante, vamos observar que função de utilidade logarítmica generalizada também satisfaz esta condição. Como vimos no item anterior a utilidade logarítmica é um caso limite da utilidade potência. A função de utilidade logarítmica generalizada $\left(U\left(V_{a}, V_{-a}\right)\right)$ é dada por:

$$
\begin{aligned}
U\left(V_{a}, V_{-a}\right) & =\gamma \ln \left(V_{a}\right)+\lambda \ln \left(\prod_{j=1 j \neq a}^{m}\left(\frac{V_{a}}{V_{j}}\right)^{\frac{1}{m-1}}\right) \\
& =\gamma \ln \left(V_{a}\right)+\lambda \ln \left(V_{a}\right)-\lambda \ln \left(\bar{V}_{-a}\right) \\
& =(\gamma+\lambda) \ln \left(V_{a}\right)-\lambda \ln \left(\bar{V}_{-a}\right)
\end{aligned}
$$

Novamente, $\bar{V}_{-a}$ é a média geométrica dos valores $V_{j}$ dos demais agentes, diferentes de $a$. Também estamos supondo que todas os valores $V_{j}, 1 \leq j \leq m$ são positivos. Note que aqui o agente $a$ tem sua "satisfação" afetada pela média geométrica dos valores dos demais agentes.

A função de utilidade logarítmica efetiva $\left(U_{e}\left(V_{a}\right)\right)$ é dada por:

$$
\begin{aligned}
U_{e}\left(V_{a}\right) & =\mathbb{E}_{\pi_{a}}\left[U\left(V_{a}, V_{-a}\right)\right] \\
& =(\gamma+\lambda) \ln \left(V_{a}\right)-\lambda \mathbb{E}_{\pi_{a}}\left[\ln \left(\bar{V}_{-a}\right)\right]
\end{aligned}
$$

Vamos calcular a primeira e a segunda derivadas da utilidade efetiva para verificar que satisfaz as condições de uma função de utilidade:

$$
\begin{gathered}
\frac{d U_{e}\left(V_{a}\right)}{d V_{a}}=\frac{(\gamma+\lambda)}{V_{a}}>0 \\
\frac{d^{2} U_{e}\left(V_{a}\right)}{d V_{a}^{2}}=-\frac{(\gamma+\lambda-1)}{V_{a}^{2}}<0
\end{gathered}
$$


O coeficiente de aversão ao risco absoluto (Arrow-Pratt) é dado por:

$$
A R_{a}\left(U_{e}\right)=-\frac{U_{e}^{\prime \prime}\left(V_{a}\right)}{U_{e}^{\prime}\left(V_{a}\right)}=\frac{1}{V_{a}}
$$

O coeficiente de aversão ao risco relativo (Arrow-Pratt) é dado por:

$$
A R_{r}\left(U_{e}\right)=V_{a} \cdot A R_{a}\left(U_{e}\right)=V_{a}\left(\frac{1}{V_{a}}\right)=1
$$

Portanto é uma função utilidade tipo CRRA ("Constant Relative Risk Aversion"), ou seja, com aversão ao risco relativa constante.

Para as funções de utilidade exponencial e quadrática, o termo perturbativo responsável pela influência social é "aditivo", isto é, a influência é causada pela média aritmética das diferenças entre os valores financeiros do agente $a$ e os demais agentes diferentes de $a$. Para as funções de utilidade potência e logarítmica , o termo perturbativo responsável pela influência social é "multiplicativo", isto é, a influência é causada pela média geométrica dos quocientes entre os valores financeiros do agente $a$ e os demais agentes diferentes de $a$. Em ambos os casos, temos que a utilidade "percebida" pelo agente $a$ é afetada pela média da expectativa dos valores financeiros dos demais. Postular esta forma de como se dá a influência social foi uma escolha guiada pelo pragmatismo para obter soluções fechadas.

Aqui cabe algumas observações, em [scheinkman2006][24], ele escreve uma utilidade com influência social na forma

$$
U\left(V_{a}, V_{-a}\right)=U\left(V_{a}\right)+\frac{1}{m-1} \sum_{j=1 j \neq a}^{m} \mathbb{E}\left[U\left(V_{j}\right)\right]+f(.)
$$

(note a presença do valor esperado da utilidade dos demais agentes, algo semelhante ao nosso véu). Porém na forma adotada, a influência causada pela utilidade dos demais é "externa", ou seja, por fora do argumento da utilidade original. Em outras palavras, a utilidade generalizada é dada pela original mais termos devidos a valores esperados da utilidade dos outros, o que torna ela separável e aditiva em relação às ações dos demais. 
No nosso caso, a riqueza percebida por pelo agente $a$ é afetada pela média da riqueza dos demais (o que torna mais adequado nossa interpretaço deste efeito como inveja) e em seguida a utilidade de $a$ é afetada.

Nos próximos capítulos utilizamos as utilidades generalizadas aqui apresentadas para desenvolver tópicos de Finanças de grande importância: assim no capítulo 4 e 5, fazemos uso das utilidades exponencial e quadrática para deduzir o CAPM com influência social. No capítulo 7 é a vez de aplicar a utilidade potência para conectar a presença de influência social e os "puzzles"EPP e RRP. 


\title{
4 Modelo CAPM Uniperiódico com Influên- cia Social - Utilidade Exponencial
}

\author{
...the anomalies uncovered in the field of Behavioral Finance are \\ interesting, but in the end he finds the behavioral approach frus- \\ trating as well. These findings are only "a collection of anomalies, \\ not a real theory. You need a theory to beat a theory", he pointed \\ out to me. A more sophisticated view is essential, one focusing \\ on the nature of the individuals and groups who compose the \\ market.
}

A. Lo (MIT) to P. Bernstein Capital Ideas Evolving

Na citação que abre este capítulo vemos uma das críticas que são feitas às pesquisas em Finanças Comportamentais, a existência de pouca ou quase nenhuma teoria que seja capaz de "dar conta" dos fenômenos observados (anomalias). Nos capítulos anteriores destacamos a existência de um viés cognitivo ("inveja") e apresentamos um "framework" para representá-lo (modelagem da presença de influência social por meio de crenças heterogêneas e funções de utilidade generalizadas). Pois bem, vamos iniciar neste capítulo a desenvolver aspectos teóricos de Finanças com o "framework" apresentado.

\subsection{Modelo do Mercado}

Neste capítulo apresentamos um modelo de apreçamento de ativos comportamental baseado em influência social. Vamos inicialmente detalhar as hipóteses do modelo:

Considere o caso uniperiódico: suponha que nosso mercado é formado por $m$ agentes e $n+1$ ativos, sendo $n$ ativos com risco (ou seja, com retorno estocástico) e 1 ativo livre de risco. Dado um agente $a$, sua alocação na carteira de ativos com risco, ou simplesmente sua alocação, é dada por

$$
\vec{X}_{a}=\left(x_{1 a}, \ldots, x_{n a}\right) \in \mathbb{R}^{n}
$$


Portanto a alocação no ativo livre é dada por $x_{0 a}=1-\sum_{k=1}^{n} x_{k a}$.

Como é usual, vamos supor que não há custos de transação para os investidores. A estrutura probabilística dos retornos dos ativos arriscados é representada por uma distribuição normal multivariada ( $\pi$ com dimensão $n$ ) com vetor de médias $\vec{\mu}_{1}=\left(r_{1}, \ldots, r_{n}\right)$ e matriz de covariâncias $\Sigma$. O retorno $R_{i}$ de um ativo $i$ é definido como

$$
R_{i}=\frac{S_{f}-S_{i}}{S_{i}}
$$

onde $S_{i}$ é o valor do ativo no instante inicial e $S_{f}$ é o valor do ativo no instante final considerados.

A riqueza de um agente no instante final é dada por:

$$
W_{a}\left(\vec{X}_{a}\right)=W_{a}^{0}\left[1+r_{f}+\sum_{k=1}^{n} x_{k a}\left(R_{k}-r_{f}\right)\right]
$$

onde $W_{a}^{0}$ é a riqueza no instante inicial e $r_{f}$ é retorno do ativo livre de risco (taxa de juros).

Em nosso modelo, a utilidade depende não apenas da riqueza do próprio agente, mas também da diferença média entre a riqueza do agente e dos demais agentes (aqui estamos capturando a influência social dos demais agentes na utilidade percebida pelo agente $a$ ). Esta influência é feita de modo perturbativo, assim temos um parâmetro $\lambda$ de controle, onde $0<\lambda<<\theta_{a}$ que representa uma perturbação em $\theta_{a}$. Vamos utilizar a função utilidade generalizada exponencial, assim a utilidade do agente $a$ é dada por

$$
U\left(W_{a}, W_{-a}\right)=-\exp \left(-\theta_{a} W_{a}-\frac{\lambda}{m-1} \sum_{j=1 j \neq a}^{m}\left(W_{a}-W_{j}\right)\right)
$$

Outra hipótese bastante importante de nosso modelo, é a crença heterogênea dos agentes em relação à alocação dos demais, isso é modelado da seguinte forma: o investidor $a$ acredita numa certa distribuição de probabilidades para a alocação dos demais agentes para cada ativo $i$.

A estrutura probabilística das crenças de $a$ quanto à alocação do ativo $i$ 
pelos demais investidores é uma distribuição normal multivariada ( $\pi_{a i} \mathrm{com}$ dimensão $m-1)$ com vetor de médias $\left(x_{i a}, \ldots, x_{i a}\right)=x_{i a} . \mathbf{1}$, onde $\mathbf{1}$ é o vetor com uns em todas as entradas e com a dimensão apropriada, e matriz de covariâncias $\Xi_{a}^{i}$, observe que a estrutura depende de cada investidor e de cada ativo. Note que não há necessidade de crenças em relação à alocação no ativo livre de risco.

A distribuição completa das crenças heterogêneas de $a, \pi_{a}$, é o produto direto das distribuições $\pi_{a i}$, ou seja:

$$
\pi_{a}=\bigotimes \pi_{a i}, 1 \leq i \leq n
$$

tendo vetor de médias $\left(x_{1 a}, \ldots, x_{1 a}, x_{2 a}, \ldots, x_{2 a}, \ldots, x_{n a}, \ldots, x_{n a}\right)$, com dimensão $n(m-1)$ e matriz de covariâncias

$$
\Xi_{a}=\left[\begin{array}{ccccc}
\Xi_{a}^{1} & \mathbb{O} & \ldots & \ldots & \mathbb{O} \\
\mathbb{O} & \ddots & \mathbb{O} & \ldots & \vdots \\
\vdots & \vdots & \Xi_{a}^{i} & \mathbb{O} & \vdots \\
\vdots & \vdots & \mathbb{O} & \ddots & \mathbb{O} \\
\mathbb{O} & \ldots & \ldots & \mathbb{O} & \Xi_{a}^{n}
\end{array}\right]
$$

de dimensão $(n(m-1))^{2}=n^{2}(m-1)^{2}$.

Note que aqui há uma simplificação, por exemplo, dados dois agentes $b$ e $c$ e os ativos $j$ e $k$, a covariância entre a crença do agente $a$ quanto a decisão do agente $b$ relativa ao ativo $j$ e a crença de $a$ quanto a decisão do agente $c$ relativa ao ativo $k$ é nula. Em outras palavras, a covariância entre ativos diferentes para agentes diferentes é nula.

Um ponto a ser ressaltado é que as crenças do agente $a$ são sobre as alocações dos demais agentes, ou seja, sobre as ações que eles vão realizar e NÃO sobre suas riquezas no instante inicial.

A adoção de estruturas probabilísticas com distribuições normais multivariadas e o uso da função de utilidade exponencial são feitas para simplificar a análise e permitir a obtenção de soluções em formas fechadas.

O problema do agente $a$ é 


$$
\max _{\overrightarrow{X_{a}}}\left\{\mathbb{E}_{R} \mathbb{E}_{\pi_{a}}\left[U\left(W_{a}, W_{-a}\right)\right]\right\}
$$

Observe que em relação a situação tradicional (sem influência social) o que temos agora, como argumento da esperança em relação à medida $\pi$ é uma esperança da utilidade com relação à crença sobre os outros agentes, isto pode ser visto como um "véu" que impede $a$ de saber exatamente como os demais agentes irão realizar suas alocações, o que por sua vez influencia na alocação de $a$.

Para calcular o termo $\mathbb{E}_{R} \mathbb{E}_{\pi_{a}}\left[U\left(W_{a}, W_{-a}\right)\right]$, devemos realizar algumas manipulações algébricas. Vamos inicialmente começar com a utilidade generalizada $U\left(W_{a}, W_{-a}\right)$ :

$$
\begin{aligned}
U\left(W_{a}, W_{-a}\right) & =-\exp \left(-\theta_{a} W_{a}-\frac{\lambda}{m-1} \sum_{j=1 j \neq a}^{m}\left(W_{a}-W_{j}\right)\right) \\
& =-\exp \left(-\left(\theta_{a}+\lambda\right) W_{a}+\frac{\lambda}{m-1} \sum_{j=1 j \neq a}^{m} W_{j}\right) \\
& =-\exp \left(-\left(\theta_{a}+\lambda\right) W_{a}^{0}\left(1+r_{f}+\sum_{k=1}^{n} x_{k a}\left(R_{k}-r_{f}\right)\right)\right) \\
& \times \exp \left(\frac{\lambda}{m-1} \sum_{j=1 j \neq a}^{m}\left[W_{j}^{0}\left(1+r_{f}+\sum_{k=1}^{n} x_{k j}\left(R_{k}-r_{f}\right)\right)\right]\right) \\
& =-\exp \left(-\left(\theta_{a}+\lambda\right) W_{a}^{0}\left(1+r_{f}\right)\right) \\
& \times \exp \left(\sum_{k=1}^{n}-\left(\theta_{a}+\lambda\right) W_{a}^{0} x_{k a}\left(R_{k}-r_{f}\right)\right) \\
& \times \exp \left(\frac{\lambda}{m-1} \sum_{j=1 j \neq a}^{m} W_{j}^{0}\left(1+r_{f}\right)\right) \\
& \times \exp \left(\frac{\lambda}{m-1} \sum_{j=1 j \neq a}^{m} W_{j}^{0}\left(\sum_{k=1}^{n} x_{k j}\left(R_{k}-r_{f}\right)\right)\right)
\end{aligned}
$$

Renomeando os termos $W_{a}^{0}\left(1+r_{f}\right)$ e $\frac{1}{m-1} \sum_{j=1 j \neq a}^{m} W_{j}^{0}\left(1+r_{f}\right)$ respectivamente, como $W_{a}^{r f}$ (representa a riqueza livre de risco do agente $a$ no instante final) 
e $\bar{W}_{-a}^{r f}$ (representa a riqueza média dos demais agentes diferentes de $a$ no instante final), obtemos

$$
\begin{aligned}
U\left(W_{a}, W_{-a}\right)= & -\exp \left(-\left(\theta_{a}+\lambda\right) W_{a}^{r f}+\lambda \bar{W}_{-a}^{r f}\right) \\
& \times \exp \left(\sum_{k=1}^{n}-\left(\theta_{a}+\lambda\right) W_{a}^{0} x_{k a}\left(R_{k}-r_{f}\right)\right) \\
& \times \exp \left(\frac{\lambda}{m-1} \sum_{k=1}^{n}\left(\left(R_{k}-r_{f}\right)\left(\vec{W}_{-a}^{0} \cdot \vec{X}_{-a}^{k}\right)\right)\right)
\end{aligned}
$$

onde

$$
\vec{W}_{-a}^{0}=\left(W_{1}^{0}, \ldots, W_{j}^{0}, \ldots, W_{m}^{0}\right)
$$

Vamos inicialmente calcular a esperança interna (integração na distribuição das crenças para cada ativo):

$$
\begin{aligned}
& \mathbb{E}_{\pi_{a}}\left[U\left(W_{a}, W_{-a}\right)\right]= \\
- & \exp \left(-\left(\theta_{a}+\lambda\right) W_{a}^{r f}+\lambda \bar{W}_{-a}^{r f}+\sum_{k=1}^{n}-\left(\theta_{a}+\lambda\right) W_{a}^{0} x_{k a}\left(R_{k}-r_{f}\right)\right) \\
\times & \int_{\mathbb{R}^{n(m-1)}}\left(D \vec{X}_{-a}^{k}\right)^{n} \exp \left(\frac{\lambda}{m-1} \sum_{k=1}^{n}\left[\left(R_{k}-r_{f}\right)\left(\vec{W}_{-a}^{0} \cdot \vec{X}_{-a}^{k}\right)\right]\right)
\end{aligned}
$$

onde

$$
\begin{aligned}
\int_{\mathbb{R}^{n(m-1)}}\left(D \vec{X}_{-a}^{k}\right)^{n} & :=\int_{\mathbb{R}^{n(m-1)}} \frac{\Pi_{k=1}^{n} d \vec{X}_{-a}^{k}}{\sqrt{(2 \pi)^{n(m-1)}\left(\Pi_{k=1}^{n} \operatorname{det} \Xi_{a}^{k}\right)}} \\
& \times \exp \left(-\frac{1}{2} \sum_{k=1}^{n}\left(\vec{X}_{-a}^{k}-x_{k a} \mathbf{1}\right) \cdot\left(\Xi_{a}^{k}\right)^{-1}\left(\vec{X}_{-a}^{k}-x_{k a} \mathbf{1}\right)\right)
\end{aligned}
$$

Vamos introduzir uma mudança de variáveis no integrando 


$$
\begin{aligned}
& \mathbb{E}_{\pi_{a}}\left[U\left(W_{a}, W_{-a}\right)\right]= \\
- & \exp \left(-\left(\theta_{a}+\lambda\right) W_{a}^{r f}+\lambda \bar{W}_{-a}^{r f}+\sum_{k=1}^{n}-\left(\theta_{a}+\lambda\right) W_{a}^{0} x_{k a}\left(R_{k}-r_{f}\right)\right) \\
\times & \exp \left(\frac{\lambda}{m-1} \sum_{k=1}^{n}\left(R_{k}-r_{f}\right)\left(\vec{W}_{-a}^{0} \cdot x_{k a} \mathbf{1}\right)\right) \\
\times & \int_{\mathbb{R}^{n(m-1)}}\left(D \vec{X}_{-a}^{k}\right)^{n} \exp \left(\frac{\lambda}{m-1} \sum_{k=1}^{n}\left(R_{k}-r_{f}\right)\left(\vec{W}_{-a}^{0} \cdot\left(\vec{X}_{-a}^{k}-x_{k a} \mathbf{1}\right)\right)\right)
\end{aligned}
$$

Note que

$$
\begin{aligned}
\frac{\lambda}{m-1} \sum_{k=1}^{n}\left(R_{k}-r_{f}\right)\left(\vec{W}_{-a}^{0} \cdot x_{k a} \mathbf{1}\right) & =\lambda \frac{\vec{W}_{-a}^{0} \cdot \mathbf{1}}{m-1} \sum_{k=1}^{n}\left(R_{k}-r_{f}\right) x_{k a} \\
& =\lambda \bar{W}_{-a}^{0} \sum_{k=1}^{n}\left(R_{k}-r_{f}\right) x_{k a}
\end{aligned}
$$

onde

$$
\bar{W}_{-a}^{0}=\frac{\vec{W}_{-a}^{0} \cdot \mathbf{1}}{m-1}
$$

Este termo representa a riqueza média dos demais agentes diferentes de $a$ no instante inicial.

Seja $\vec{R}-r_{f} \mathbf{1}=\left(R_{1}-r_{f}, \ldots, R_{n}-r_{f}\right) \operatorname{logo} \sum_{k=1}^{n} x_{k a}\left(R_{k}-r_{f}\right)=\left(\vec{R}-r_{f} \mathbf{1}\right) \cdot \vec{X}_{a}$ assim, 


$$
\begin{aligned}
& \sum_{k=1}^{n}-\left(\theta_{a}+\lambda\right) W_{a}^{0} x_{k a}\left(R_{k}-r_{f}\right)+\frac{\lambda}{m-1} \sum_{k=1}^{n}\left(\left(R_{k}-r_{f}\right)\left(\vec{W}_{-a}^{0} \cdot x_{k a} \mathbf{1}\right)\right. \\
= & -\left(\theta_{a}+\lambda\right) W_{a}^{0} \sum_{k=1}^{n} x_{k a}\left(R_{k}-r_{f}\right)+\lambda \bar{W}_{-a}^{0} \sum_{k=1}^{n} x_{k a}\left(R_{k}-r_{f}\right) \\
= & \left(-\theta_{a} W_{a}^{0}-\lambda\left(W_{a}^{0}-\bar{W}_{-a}^{0}\right)\right) \sum_{k=1}^{n} x_{k a}\left(R_{k}-r_{f}\right) \\
= & \left(-\theta_{a} W_{a}^{0}-\lambda\left(W_{a}^{0}-\bar{W}_{-a}^{0}\right)\right)\left(\vec{R}-r_{f} \mathbf{1}\right) \cdot \vec{X}_{a}
\end{aligned}
$$

Temos

$$
\begin{aligned}
& \int_{\mathbb{R}^{n(m-1)}}\left(D \vec{X}_{-a}^{k}\right)^{n} \exp \left(\frac{\lambda}{m-1} \sum_{k=1}^{n}\left(R_{k}-r_{f}\right)\left(\vec{W}_{-a}^{0} \cdot\left(\vec{X}_{-a}^{k}-x_{k a} \mathbf{1}\right)\right)\right) \\
= & \exp \left(-\frac{1}{2} \sum_{k=1}^{n}\left(\frac{\lambda}{m-1}\left(R_{k}-r_{f}\right) \vec{W}_{-a}^{0}\right) \cdot \Xi_{a}^{k}\left(\frac{\lambda}{m-1}\left(R_{k}-r_{f}\right) \vec{W}_{-a}^{0}\right)\right) \\
= & \exp \left(-\frac{1}{2}\left(\frac{\lambda}{m-1}\right)^{2} \sum_{k=1}^{n}\left(R_{k}-r_{f}\right)^{2} \vec{W}_{-a}^{0} \cdot \Xi_{a}^{k} \vec{W}_{-a}^{0}\right) \\
= & \exp \left(-\frac{1}{2}\left(\frac{\lambda}{m-1}\right)^{2} \sum_{k=1}^{n}\left(R_{k}-r_{f}\right)^{2} w_{k}\right)
\end{aligned}
$$

onde $w_{k}=\vec{W}_{-a}^{0} \cdot \Xi_{a}^{k} \vec{W}_{-a}^{0}>0$, este termo pode ser visto como a norma ao quadrado do vetor $\vec{W}_{-a}^{0}$ considerando $\Xi_{a}^{k}$ como matriz de pesos do produto interno.

Considere as matrizes elementares $E_{i j} \operatorname{com}\left(E_{i j}\right)=\delta_{i j}$ onde $\delta_{i j}$ é o delta de Kronecker, podemos reescrever o termo $\sum_{k=1}^{n}\left(R_{k}-r_{f}\right)^{2} w_{k}$ como $(\vec{R}-$ $\left.r_{f} \mathbf{1}\right) \mathbb{W}\left(\vec{R}-r_{f} \mathbf{1}\right)$ onde $\mathbb{W}=\sum_{k=1}^{n} w_{k} E_{k k}, \operatorname{logo}$

$$
\begin{aligned}
& \exp \left(-\frac{1}{2}\left(\frac{\lambda}{m-1}\right)^{2} \sum_{k=1}^{n}\left(R_{k}-r_{f}\right)^{2} w_{k}\right) \\
= & \exp \left(-\frac{1}{2}\left(\frac{\lambda}{m-1}\right)^{2}\left(\vec{R}-r_{f} \mathbf{1}\right) \mathbb{W}\left(\vec{R}-r_{f} \mathbf{1}\right)\right)
\end{aligned}
$$


Finalmente:

$$
\begin{aligned}
\mathbb{E}_{\pi_{a}}\left[U\left(W_{a}, W_{-a}\right)\right]= & -\exp \left(-\left(\theta_{a}+\lambda\right) W_{a}^{r f}+\lambda \bar{W}_{-a}^{r f}\right) \\
& \times \exp \left(\left(-\theta_{a} W_{a}^{0}-\lambda\left(W_{a}^{0}-\bar{W}_{-a}^{0}\right)\right)\left(\vec{R}-r_{f} \mathbf{1}\right) \cdot \vec{X}_{a}\right) \\
& \times \exp \left(-\frac{1}{2}\left(\frac{\lambda}{m-1}\right)^{2}\left(\vec{R}-r_{f} \mathbf{1}\right) \mathbb{W}\left(\vec{R}-r_{f} \mathbf{1}\right)\right)
\end{aligned}
$$

A matriz $\mathbb{W}$ carrega toda a informação sobre as matrizes de covariâncias de $a$ sobre a alocação dos demais agentes em relação aos ativos arriscados. Observe que o termo onde $\mathbb{W}$ aparece é de segunda ordem ( $\lambda$ está ao quadrado), enquanto os demais termos termos com $\lambda$ são de primeira ordem; isto indica que a importância deste termo deve ser muito menor que os demais (pois $\lambda$ é um termo perturbativo e $\theta_{a}>>>0$ ). Na verdade, conforme veremos sua importância é nula no equilíbrio, o que constitui algo surpreendente.

Queremos calcular $\mathbb{E}_{R} \mathbb{E}_{\pi_{a}}\left[U\left(W_{a}, W_{-a}\right)\right]$ :

$$
\begin{aligned}
& \mathbb{E}_{R} \mathbb{E}_{\pi_{a}}\left[U\left(W_{a}, W_{-a}\right)\right]= \\
- & \exp \left(-\left(\theta_{a}+\lambda\right) W_{a}^{r f}+\lambda \bar{W}_{-a}^{r f}\right) \\
\times & \int_{\mathbb{R}^{n}}(D \vec{R}) \exp \left(-\theta_{a} W_{a}^{0}-\lambda\left(W_{a}^{0}-\bar{W}_{-a}^{0}\right)\left(\vec{R}-r_{f} \mathbf{1}\right) \cdot \vec{X}_{a}\right) \\
& \times \exp \left(-\frac{1}{2}\left(\frac{\lambda}{m-1}\right)^{2}\left(\vec{R}-r_{f} \mathbf{1}\right) \mathbb{W}\left(\vec{R}-r_{f} \mathbf{1}\right)\right)
\end{aligned}
$$

onde

$$
\int_{\mathbb{R}^{n}}(D \vec{R}):=\int_{\mathbb{R}^{n}} \frac{d \vec{R}}{\sqrt{(2 \pi)^{n} \operatorname{det} \Sigma}} \exp \left(-\frac{1}{2}\left(\vec{R}-\vec{\mu}_{1}\right) \Sigma^{-1}\left(\vec{R}-\vec{\mu}_{1}\right)^{\prime}\right)
$$

Fazendo $\vec{R}-\vec{\mu}_{1}=\left(\vec{R}-r_{f} \mathbf{1}\right)+\left(r_{f} \mathbf{1}-\vec{\mu}_{1}\right)$, obtemos 


$$
\begin{aligned}
& \mathbb{E}_{R} \mathbb{E}_{\pi_{a}}\left[U\left(W_{a}, W_{-a}\right)\right]= \\
- & \exp \left(-\left(\theta_{a}+\lambda\right) W_{a}^{r f}+\lambda \bar{W}_{-a}^{r f}-\frac{1}{2}\left(r_{f} \mathbf{1}-\vec{\mu}_{1}\right) \Sigma^{-1}\left(r_{f} \mathbf{1}-\vec{\mu}_{1}\right)^{\prime}\right) \\
\times & \left.\left.\int_{\mathbb{R}^{n}}\left(D \vec{R}^{\prime}\right) \exp \left(-\theta_{a} W_{a}^{0}-\lambda\left(W_{a}^{0}-\bar{W}_{-a}^{0}\right) \vec{X}_{a}\right)-\left(r_{f} \mathbf{1}-\vec{\mu}_{1}\right) \Sigma^{-1}\right) \cdot\left(\vec{R}-r_{f} \mathbf{1}\right)\right) \\
& \times \exp \left(-\frac{1}{2}\left(\frac{\lambda}{m-1}\right)^{2}\left(\vec{R}-r_{f} \mathbf{1}\right) \mathbb{W}\left(\vec{R}-r_{f} \mathbf{1}\right)\right)
\end{aligned}
$$

onde

$$
\int_{\mathbb{R}^{n}}\left(D \vec{R}^{\prime}\right)=\int_{\mathbb{R}^{n}} \frac{d \vec{R}}{\sqrt{(2 \pi)^{n} \operatorname{det} \Sigma}} \exp \left(-\frac{1}{2}\left(\vec{R}-r_{f} \mathbf{1}\right) \Sigma^{-1}\left(\vec{R}-r_{f} \mathbf{1}\right)^{\prime}\right)
$$

Temos

$$
\begin{aligned}
& \int_{\mathbb{R}^{n}}\left(D \vec{R}^{\prime}\right) \exp \left(\left(-\theta_{a} W_{a}^{0}-\lambda\left(W_{a}^{0}-\bar{W}_{-a}^{0}\right) \vec{X}_{a}-\left(r_{f} \mathbf{1}-\vec{\mu}_{1}\right) \Sigma^{-1}\right)\left(\vec{R}-r_{f} \mathbf{1}\right)\right) \\
& \times \exp \left(-\frac{1}{2}\left(\frac{\lambda}{m-1}\right)^{2}\left(\vec{R}-r_{f} \mathbf{1}\right) \mathbb{W}\left(\vec{R}-r_{f} \mathbf{1}\right)\right)= \\
& \int_{\mathbb{R}^{n}} \frac{d \vec{R}}{\sqrt{(2 \pi)^{n} \operatorname{det} \Sigma}} \exp \left(\left(-\theta_{a} W_{a}^{0}-\lambda\left(W_{a}^{0}-\vec{W}_{-a}^{0}\right) \vec{X}_{a}\right) \cdot\left(\vec{R}-r_{f} \mathbf{1}\right)\right) \\
& \times \exp \left(\left(-\left(r_{f} \mathbf{1}-\vec{\mu}_{1}\right) \Sigma^{-1}\right) \cdot\left(\vec{R}-r_{f} \mathbf{1}\right)\right) \\
& \left.\times \exp \left(-\frac{1}{2}\left(\vec{R}-r_{f} \mathbf{1}\right)\left(\left(\frac{\lambda}{m-1}\right)^{2} \mathbb{W}+\Sigma^{-1}\right) \vec{R}-r_{f} \mathbf{1}\right)^{\prime}\right)
\end{aligned}
$$

Assim, temos 


$$
\begin{aligned}
& \mathbb{E}_{R} \mathbb{E}_{\pi_{a}}\left[U\left(W_{a}, W_{-a}\right)\right]= \\
- & \exp \left(-\left(\theta_{a}+\lambda\right) W_{a}^{r f}+\lambda \bar{W}_{-a}^{r f}-\frac{1}{2}\left(r_{f} \mathbf{1}-\vec{\mu}_{1}\right) \Sigma^{-1}\left(r_{f} \mathbf{1}-\vec{\mu}_{1}\right)^{\prime}\right) \\
\times & \exp -\frac{1}{2}\left(-\theta_{a} W_{a}^{0}-\lambda\left(W_{a}^{0}-\bar{W}_{-a}^{0}\right) \vec{X}_{a}-\left(r_{f} \mathbf{1}-\vec{\mu}_{1}\right) \Sigma^{-1}\right) \\
\times & \left(\left(\frac{\lambda}{m-1}\right)^{2} \mathbb{W}+\Sigma^{-1}\right)^{-1} \\
\times & \left(-\theta_{a} W_{a}^{0}-\lambda\left(W_{a}^{0}-\bar{W}_{-a}^{0}\right) \vec{X}_{a}-\left(r_{f} \mathbf{1}-\vec{\mu}_{1}\right) \Sigma^{-1}\right)
\end{aligned}
$$

Vamos fazer $-\left(r_{f} \mathbf{1}-\vec{\mu}_{1}\right)=\vec{r}_{e},-\theta_{a} W_{a}^{0}-\lambda\left(W_{a}^{0}-\bar{W}_{-a}^{0}\right)=\ln \left(-U_{a}^{0}\right),-\left(\theta_{a}+\right.$ $\lambda) W_{a}^{r f}+\lambda \bar{W}_{-a}^{r_{f}}=\ln \left(-U_{a}^{r_{f}}\right)$ e $\left(\left(\frac{\lambda}{m-1}\right)^{2} \mathbb{W}+\Sigma^{-1}\right)^{-1}=\mathbb{M}$. Assim, teremos:

$$
\begin{aligned}
& \mathbb{E}_{R} \mathbb{E}_{\pi_{a}}\left[U\left(W_{a}, W_{-a}\right)\right]= \\
- & \exp \left(\ln \left(-U_{a}^{r_{f}}\right)-\frac{1}{2} \vec{r}_{e} \Sigma^{-1} \vec{r}_{e}^{\prime}\right) \\
\times & \exp \left(-\frac{1}{2}\left[\ln \left(-U_{a}^{0}\right) \vec{X}_{a}+\vec{r}_{e} \Sigma^{-1}\right] \mathbb{M}\left[\ln \left(-U_{a}^{0}\right) \vec{X}_{a}+r_{e} \Sigma^{-1}\right]^{\prime}\right)
\end{aligned}
$$

Vamos calcular o gradiente da função utilidade, assim temos:

$$
\begin{aligned}
& \frac{\partial}{\partial \vec{X}_{a}}\left(\mathbb{E}_{R} \mathbb{E}_{\pi_{a}}\left[U\left(W_{a}, W_{-a}\right)\right]\right)= \\
& \left(-\frac{1}{2}\left[\ln \left(-U_{a}^{0}\right) \mathbb{M}\left(\ln \left(-U_{a}^{0}\right) \vec{X}_{a}+\vec{r}_{e} \Sigma^{-1}\right)\right]\right) \\
+ & \left.\left(-\frac{1}{2}\left[\ln \left(-U_{a}^{0}\right) \vec{X}_{a}+\vec{r}_{e} \Sigma^{-1}\right) \mathbb{M} \ln \left(-U_{a}^{0}\right)\right]\right) \\
\times & \mathbb{E}_{R} \mathbb{E}_{\pi_{a}}\left[U\left(W_{a}, W_{-a}\right)\right]= \\
- & \ln \left(-U_{a}^{0}\right) \mathbb{M}\left(\ln \left(-U_{a}^{0}\right) \vec{X}_{a}+\vec{r}_{e} \Sigma^{-1}\right) \mathbb{E}_{R} \mathbb{E}_{\pi_{a}}\left[U\left(W_{a}, W_{-a}\right)\right]
\end{aligned}
$$


No equilíbrio devemos ter o gradiente nulo (condições de primeira ordem):

$$
\begin{aligned}
\frac{\partial}{\partial \vec{X}_{a}}\left(\mathbb{E}_{R} \mathbb{E}_{\pi_{a}}\left[U\left(W_{a}, W_{-a}\right)\right]\right)=\overrightarrow{0} & \Leftrightarrow \ln \left(-U_{a}^{0}\right) \mathbb{M}\left(\ln \left(-U_{a}^{0}\right) \vec{X}_{a}^{*}+\vec{r}_{e} \Sigma^{-1}\right)=\overrightarrow{0} \\
\Leftrightarrow \ln \left(-U_{a}^{0}\right) \mathbb{M} \vec{X}_{a}^{*}+\mathbb{M} \vec{r}_{e} \Sigma^{-1}=\overrightarrow{0} & \\
\Leftrightarrow \vec{X}_{a}^{*}= & \frac{\vec{r}_{e} \Sigma^{-1}}{-\ln \left(-U_{a}^{0}\right)} \\
\vec{X}_{a}^{*} & =\frac{(55)}{\theta_{a} W_{a}^{0}+\lambda\left(W_{a}^{0}-\bar{W}_{-a}^{0}\right)}
\end{aligned}
$$

Note um fato muito importante, a matriz $\mathbb{M}=\left(\left(\frac{\lambda}{m-1}\right)^{2} \mathbb{W}+\Sigma^{-1}\right)^{-1}$ não aparece na solução de equilíbrio, o que significa que a matriz $\mathbb{W}$ também não, ou seja, as expectativas (crenças heterogêneas) que o agente $a$ tem sobre como os demais investidores alocam os ativos arriscados só importa quanto ao valor esperado destas alocações (valor esperado segundo o "véu") e não em relação as covariâncias (momento de segunda ordem) e demais momentos.

Observe também que na solução de equilíbrio, a presença do termo $\lambda\left(W_{a}^{0}-\right.$ $\bar{W}_{-a}^{0}$ ) está associada a presença de influência social, tomando $\lambda=0$ recuperamos a situação clássica.

Em particular, (omitindo somatórios quando há índices repetidos, também conhecida como notação de Einstein)

$$
x_{a i}^{*}=\frac{\left(r_{k}-r_{f}\right) \Sigma_{k i}^{-1}}{-\ln \left(-U_{a}^{0}\right)}
$$

Por outro lado,

$$
\vec{X}_{a}^{*}=\frac{\vec{r}_{e} \Sigma^{-1}}{-\ln \left(-U_{a}^{0}\right)} \Leftrightarrow
$$




$$
\vec{r}_{e}=-\ln \left(-U_{a}^{0}\right) \vec{X}_{a}^{*} \Sigma
$$

Em particular, (omitindo somatórios quando há índices repetidos)

$$
\begin{aligned}
r_{i}-r_{f} & =-\ln \left(-U_{a}^{0}\right) x_{a l}^{*} \Sigma_{l i} \\
& =-\ln \left(-U_{a}^{0}\right) \frac{\left(r_{k}-r_{f}\right) \Sigma_{k l}^{-1}}{-\ln \left(-U_{a}^{0}\right)} \Sigma_{l i} \\
& =\left(r_{k}-r_{f}\right) \Sigma_{k l}^{-1} \Sigma_{l i}
\end{aligned}
$$

\subsection{CAPM com Influência Social}

Vamos agora, em busca de uma relação entre os retornos esperados dos ativos

e o do mercado como no CAPM tradicional, só que agora em nosso modelo.

Por definição, temos $W_{0}^{M}=\sum_{i=1}^{n} W_{a}^{0}$. Vamos partir da seguinte identidade:

$$
\begin{aligned}
x_{a i}^{*}= & \frac{\left(r_{k}-r_{f}\right) \Sigma_{k i}^{-1}}{-\ln \left(-U_{a}^{0}\right)} \Rightarrow \\
\sum_{i=1}^{n} \frac{W_{a}^{0}}{W_{M}^{0}} x_{a i}^{*}\left(r_{i}-r_{f}\right) & =\sum_{i=1}^{n} \frac{W_{a}^{0}\left(r_{k}-r_{f}\right) \Sigma_{k i}^{-1}\left(r_{i}-r_{f}\right)}{-W_{M}^{0} \ln \left(-U_{a}^{0}\right)} \\
& =\frac{W_{a}^{0} \vec{r}_{e} \Sigma^{-1} \vec{r}_{e}}{-W_{M}^{0} \ln \left(-U_{a}^{0}\right)}
\end{aligned}
$$

Somando esta identidade para todos os agentes do mercado obtemos:

$$
r_{M}-r_{f}=\frac{\vec{r}_{e} \Sigma^{-1} \vec{r}_{e}}{W_{M}^{0}} \sum_{a=1}^{m} \frac{W_{a}^{0}}{-\ln \left(-U_{a}^{0}\right)}
$$


Vamos olhar mais detidamente o último termo:

$$
\begin{aligned}
\sum_{a=1}^{m} \frac{W_{a}^{0}}{-\ln \left(-U_{a}^{0}\right)} & =\sum_{a=1}^{m} \frac{W_{a}^{0}}{\left(\theta_{a}+\lambda\right) W_{a}^{0}-\lambda \bar{W}_{-a}^{0}} \\
& =\sum_{a=1}^{m} \frac{1}{\left(\theta_{a}+\lambda\right)-\lambda \frac{\bar{W}_{-a}^{0}}{W_{a}^{0}}} \\
& =\sum_{a=1}^{m} \frac{1}{\theta_{a}+\lambda\left(1-\frac{\bar{W}_{-a}^{0}}{W_{a}^{0}}\right)}
\end{aligned}
$$

Comparando com o CAPM tradicional, é razoável denominar este termo como o inverso da aversão ao risco do mercado efetiva e denotá-lo por $\frac{1}{\theta_{M}}$. Observe que se fizermos $\lambda=0$ o que corresponde a ausência de influência social, recuperamos o caso tradicional. Assim,

$$
r_{M}-r_{f}=\frac{\vec{r}_{e} \Sigma^{-1} \vec{r}_{e}}{W_{M}^{0} \theta_{M}}
$$

Note que $W_{M}^{0}$ tem ordem de grandeza $m$, sua unidade de medida é a moeda utilizada, enquanto $\theta_{M}$ tem ordem de grandeza $1 / \mathrm{m}$ sua unidade de medida é o inverso da moeda utilizada, logo seu produto é adimensional, como esperado.

No equilíbrio, temos

$$
R_{M}=\sum_{a=1}^{m}\left[\frac{W_{a}^{0}}{W_{M}^{0}}\left(r_{f}+\sum_{j=1}^{n} x_{a j}^{*}\left(R_{j}-r_{f}\right)\right)\right]
$$

$\log 0$ : 


$$
\begin{aligned}
\operatorname{cov}_{R}\left[R_{i}, R_{M}\right] & =\operatorname{cov}_{R}\left[R_{i}, \sum_{a=1}^{m}\left[\frac{W_{a}^{0}}{W_{M}^{0}}\left(r_{f}+\sum_{j=1}^{n} x_{a j}^{*}\left(R_{j}-r_{f}\right)\right)\right]\right] \\
& =\sum_{a=1}^{m}\left(\frac{W_{a}^{0}}{W_{M}^{0}}\left[\sum_{j=1}^{n} x_{a j}^{*} \operatorname{cov}_{R}\left[R_{i}, R_{j}\right]\right]\right) \\
& =\sum_{a=1}^{m}\left(\frac{W_{a}^{0}}{W_{M}^{0}}\left[\sum_{j=1}^{n} x_{a j}^{*} \Sigma_{i j}\right]\right) \\
& =\left(\sum_{a=1}^{m} \frac{W_{a}^{0}}{\theta_{a} W_{a}^{0}+\lambda\left(W_{a}^{0}-\bar{W}_{-a}^{0}\right)}\right) \frac{\left(r_{k}-r_{f}\right) \Sigma_{k j}^{-1} \Sigma_{i j}}{W_{M}^{0}} \\
& =\frac{r_{i}-r_{f}}{W_{M}^{0} \theta_{M}}
\end{aligned}
$$

Temos também:

$$
\begin{aligned}
\sigma_{M}^{2} & =\operatorname{cov}_{R}\left[R_{M}, R_{M}\right] \\
& =\operatorname{cov}_{R}\left[R_{M}, \sum_{a=1}^{m}\left(\frac{W_{a}^{0}}{W_{M}^{0}}\left[r_{f}+\sum_{i=1}^{n} x_{a i}^{*}\left(R_{i}-r_{f}\right)\right]\right)\right] \\
& =\sum_{a=1}^{m}\left(\frac{W_{a}^{0}}{W_{M}^{0}}\left[\sum_{i=1}^{n} x_{a i}^{*} \operatorname{cov}_{R}\left[R_{i}, R_{M}\right]\right]\right) \\
& =\sum_{a=1}^{m}\left(\frac{W_{a}^{0}}{W_{M}^{0}}\left[\frac{\left(r_{k}-r_{f}\right) \Sigma_{k i}^{-1}}{\theta_{a} W_{a}^{0}+\lambda\left(W_{a}^{0}-\bar{W}_{-a}^{0}\right)} \frac{r_{i}-r_{f}}{W_{M}^{0} \theta_{M}}\right]\right) \\
& =\sum_{a=1}^{m}\left(\frac{W_{a}^{0}}{\theta_{a} W_{a}^{0}+\lambda\left(W_{a}^{0}-\bar{W}_{-a}^{0}\right)}\right) \frac{\left(r_{k}-r_{f}\right) \Sigma_{k i}^{-1}\left(r_{i}-r_{f}\right)}{\left(W_{M}^{0}\right)^{2} \theta_{M}} \\
& \Leftrightarrow \sigma_{M}^{2}=\frac{\overrightarrow{r_{e}} \Sigma^{-1} \overrightarrow{r_{e}}}{\left(W_{M}^{0}\right)^{2}\left(\theta_{M}\right)^{2}} \Leftrightarrow \sigma_{M}=\frac{\sqrt{r_{e} \Sigma^{-1}} \overrightarrow{r_{e}}}{W_{M}^{0}} \frac{1}{\theta_{M}}
\end{aligned}
$$

onde $\sigma_{M}$ é a volatilidade do mercado. Assim, podemos observar que a volatilidade do mercado $\left(\sigma_{M}\right)$ é inversamente proporcional à aversão ao risco efetiva do mercado $\left(\theta_{M}\right)$. Isso já ocorria no modelo tradicional, porém aqui, como $\theta_{M}$ está relacionado com $\lambda$, que captura o efeito de influência social, 
podemos esperar obter conclusões interessantes, ligando a volatilidade do mercado com influência social.

Agora, vamos relacionar $r_{M}-r_{f} \operatorname{com} r_{i}-r_{f}$ :

Inicialmente observe que:

$$
\begin{aligned}
\sigma_{M}^{2}=\operatorname{cov}_{R}\left[R_{M}, R_{M}\right] & =\frac{\overrightarrow{r_{e} \Sigma^{-1}} \overrightarrow{r_{e}}}{\left(W_{M}^{0}\right)^{2}\left(\theta_{M}\right)^{2}}=\frac{r_{M}-r_{f}}{W_{M}^{0} \theta_{M}} \Leftrightarrow \\
W_{M}^{0} \theta_{M} & =\frac{r_{M}-r_{f}}{\operatorname{cov}_{R}\left[R_{M}, R_{M}\right]}
\end{aligned}
$$

Temos também que

$$
\begin{aligned}
\operatorname{cov}_{R}\left[R_{i}, R_{M}\right] & =\frac{r_{i}-r_{f}}{W_{M}^{0} \theta_{M}} \Leftrightarrow \\
W_{M}^{0} \theta_{M} & =\frac{r_{i}-r_{f}}{\operatorname{cov}_{R}\left[R_{i}, R_{M}\right]}
\end{aligned}
$$

Por outro lado,

$$
\begin{aligned}
\frac{r_{M}-r_{f}}{\operatorname{cov}_{R}\left[R_{M}, R_{M}\right]} & =\frac{r_{i}-r_{f}}{\operatorname{cov}_{R}\left[R_{i}, R_{M}\right]} \Leftrightarrow \\
r_{i}-r_{f} & =\frac{\operatorname{cov}_{R}\left[R_{i}, R_{M}\right]}{\operatorname{cov}_{R}\left[R_{M}, R_{M}\right]}\left(r_{M}-r_{f}\right)
\end{aligned}
$$

Fazendo $\beta_{i}=\frac{\operatorname{cov}_{R}\left[R_{i}, R_{M}\right]}{\operatorname{cov}_{R}\left[R_{M}, R_{M}\right]}$, obtemos o resultado desejado:

Teorema: (CAPM com influência social) O excesso de retorno de cada ativo é diretamente proporcional ao excesso de retorno do mercado, onde o coeficiente de proporcionalidade $\beta_{i}$ está relacionado à presença da influência social, algo não presente no caso tradicional. 


$$
r_{i}-r_{f}=\beta_{i}\left(r_{M}-r_{f}\right)
$$

Onde,

$$
\begin{aligned}
\beta_{i} & =\frac{\operatorname{cov}_{R}\left[R_{i}, R_{M}\right]}{\operatorname{cov}_{R}\left[R_{M}, R_{M}\right]} \\
& =\frac{W_{M}^{0} \theta_{M}\left(r_{i}-r_{f}\right)}{\vec{r}^{\Sigma^{-1}} \overrightarrow{r_{e}}}
\end{aligned}
$$

Como vimos anteriormente

$$
\theta_{M}=\left(\sum_{a=1}^{m} \frac{1}{\theta_{a}+\lambda\left(1-\frac{\bar{W}_{-a}^{0}}{W_{a}^{0}}\right)}\right)^{-1}
$$

De modo explícito, em palavras, temos que $\beta_{i}$ depende de $\theta_{M}$ que por sua vez incorpora a presença de influência social o que representa uma novidade em relação a situação tradicional.

\subsection{Analisando Algumas Consequências}

Vamos investigar a influência da aversão ao risco do mercado efetiva em nossa fórmula e comparar com a situação no modelo tradicional. A partir de agora vamos realizar a seguinte simplificação: vamos considerar o termo $\theta_{a}$ como sendo igual para todos os agentes, assim $\theta_{a}=\theta$ para todos os agentes $a$, $1 \leq a \leq m$.

\section{Temos:}

$$
\frac{1}{\theta_{M}}=\sum_{a=1}^{m}\left(\frac{1}{\theta+\lambda\left(1-\frac{\bar{W}_{-a}^{0}}{W_{a}^{0}}\right)}\right)
$$


Para $m \rightarrow \infty$, pela Lei dos Grandes Números, temos:

$$
\begin{aligned}
& \frac{1}{\frac{\theta_{M}}{m}}=\frac{\sum_{a=1}^{m}\left(\frac{1}{\theta+\lambda\left(1-\frac{\bar{W}_{-a}^{0}}{W_{a}^{0}}\right)}\right)}{m} \Rightarrow \\
& \frac{1}{\theta_{m}}=\int_{\mathbb{R}} \frac{f(W) d W}{\theta+\lambda\left(1-\frac{\mathbb{E}_{W}[W]}{W}\right)}
\end{aligned}
$$

Onde $\theta_{m}$ é a aversão ao risco média efetiva. Na última integral, a fim de simplificar a notação, efetuamos um abuso de linguagem: $W$ representa a riqueza de um agente qualquer no instante inicial.

Tomando $W \sim \operatorname{Pareto}\left(\alpha, W_{\min }\right)$ (onde $\alpha>1$ é um parâmetro de "shape" da distribuição e se relaciona é distribuição de riqueza e $W_{\min }$ significa uma riqueza mínima) temos:

$f(W)=\frac{\alpha}{W_{\min }}\left(\frac{W_{\min }}{W}\right)^{\alpha+1}$, se $W \geq W_{\min }$ e $f(W)=0$, se $W<W_{\min }$

com

$$
\mathbb{E}_{W}[W]=\frac{\alpha W_{\min }}{\alpha-1}
$$

Assim, teremos:

$$
\frac{1}{\theta_{m}}=\int_{W_{\min }}^{+\infty} \frac{\frac{\alpha}{W_{\min }}\left(\frac{W_{\min }}{W}\right)^{\alpha+1} d W}{\theta+\lambda\left(1-\frac{\mathbb{E}_{W}[W]}{W}\right)}
$$

Manipulando o último termo, obtemos: 


$$
\begin{aligned}
\frac{1}{\theta_{m}} & =\alpha(\alpha-1) W_{\min }^{\alpha} \int_{W_{\min }}^{+\infty} \frac{d W}{(\theta+\lambda)(\alpha-1) W^{\alpha+1}-\alpha \lambda W_{\min } W^{\alpha}} \\
& =\alpha(\alpha-1) W_{\min }^{\alpha} \int_{W_{\min }}^{+\infty} \frac{d W}{\left((\theta+\lambda)(\alpha-1) W-\alpha \lambda W_{\min }\right) W^{\alpha}} \\
& =\alpha(\alpha-1) \int_{1}^{+\infty} \frac{d W}{((\theta+\lambda)(\alpha-1) W-\alpha \lambda) W^{\alpha}}
\end{aligned}
$$

O índice de Gini (denotado por $G$ ) é um indicador bem conhecido em Economia para medir o nível de desigualdade (igualdade) de riqueza, ele varia entre 0 e 1 , onde $G=0$ significa igualdade total de distribuição de riqueza e $G=1$ significa completa desigualdade de distribuição de riqueza. Se a distribuição de riqueza segue a distribuição de Pareto: $W \sim \operatorname{Pareto}\left(\alpha, W_{\min }\right)$ (esta distribuição é usual para modelar distribuições de renda e riqueza), o parâmetro $\alpha$ pode ser relacionado ao índice de Gini, temos:

$$
G=\frac{1}{2 \alpha-1} \Leftrightarrow \alpha=\frac{1+G}{2 G}
$$

Note que $1<\alpha+\infty \Leftrightarrow 0<G<1$, assim, o indicador não assume os valores 0 e 1, os quais devem analisados ser por meio de tendências (comportamento assintótico em direção aos extremos).

Podemos relacionar explicitamente o índice de Gini e a aversão ao risco média, assim, sem fazer uso de aproximações temos (forma exata):

\section{Teorema: Fórmula da aversão ao risco média efetiva do mercado} Em nosso modelo, considerando as hipóteses feitas sobre o mercado, com a influência social representada pela função de utilidade generalizada exponencial e a estrutura de crenças heterogêneas representada por uma distribuição normal multivariada, e ainda com a distribuição de riqueza dos agentes dada por uma Pareto de indice de Gini $G,(0<G<1)$ temos que a aversão ao risco média efetiva do mercado é:

$$
\frac{1}{\theta_{m}}=\frac{1-G^{2}}{2 G} \int_{1}^{+\infty} \frac{d W}{((\theta+\lambda)(1-G) W-\lambda(1+G)) W^{\frac{1+G}{2 G}}}
$$




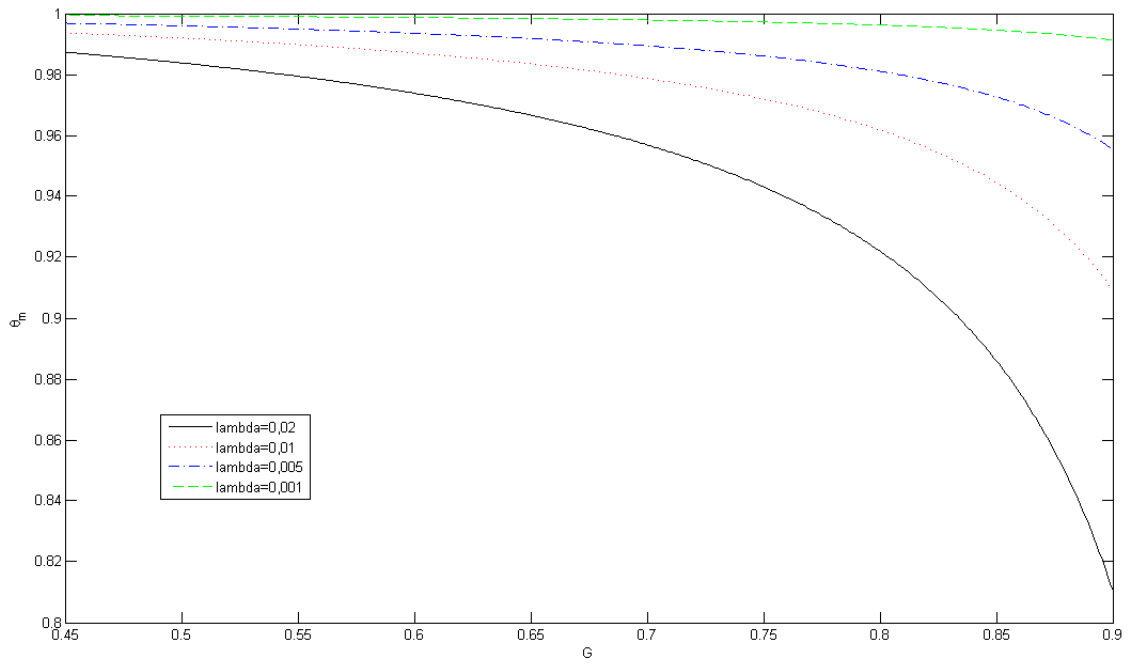

Figura 1: Aversão ao risco média efetiva $\left(\theta_{m}\right)$ versus índice de Gini $(\mathrm{G})$ Utilidade Exponencial

Esta integral pode ser calculada numericamente. Assim, tomando como valor base $\theta=1$ da aversão ao risco dos agentes, podemos observar de maneira gráfica a relação entre a aversão ao risco média efetiva do mercado $\theta_{m} \mathrm{em}$ função do índice de Gini $G$ na figura 1, para alguns valores do parâmetro perturbativo $(\lambda)$.

A partir da análise dos gráficos podemos relacionar $\theta_{m} \operatorname{com} \alpha$ e $G$. Tomando

os parâmetros $\theta$ e $\lambda$ constantes temos: aumento da desigualdade de riqueza $\Leftrightarrow G \searrow 1 \Leftrightarrow \alpha \nearrow+\infty \Rightarrow \theta_{m} \nearrow$, ou seja, um aumento da desigualdade de riqueza dos investidores (nossa população está restrita aos participantes do mercado) leva a uma diminuição da aversão ao risco média.

$\mathrm{Na}$ situação tradicional (tomando $\lambda=0$ ) a aversão ao risco de um agente $a$ é $A R\left(W_{a}\right)=-U^{\prime \prime}\left(W_{a}\right) / U^{\prime}\left(W_{a}\right)=\theta$, como todos os agentes têm a mesma aversão ao risco, a aversão ao risco do mercado média $\left(\theta_{m}\right)$ é $\theta$. Porém em nosso modelo $(\lambda>0)$ conforme os gráficos em todos os casos, o que observamos é que $\theta_{m}$ é menor que o do modelo tradicional (para qualquer $G$ e menor fica quando $G$ aumenta), ou seja, influência social leva à diminuição da aversão ao risco do mercado em relação ao modelo tradicional. 
Na equação que relaciona $r_{M}-r_{f} \operatorname{com} \theta_{M}$, temos que apenas $r_{M}$ e $\theta_{M}$ são endógenas, mais ainda, se $\theta_{M}$ diminui, $r_{M}-r_{f}$ aumenta o que só pode ocorrer se $r_{M}$ aumenta. Temos uma previsão de que um $\theta_{M}$ pequeno (menor que o tradicional) leva a um aumento do excesso de retorno do mercado.

Como sabemos, a volatilidade do mercado $\left(\sigma_{M}\right)$ é inversamente proporcional à aversão ao risco efetiva do mercado $\left(\theta_{M}\right)$, logo um aumento da desigualdade de riqueza dos investidores leva a um aumento da volatilidade do mercado (de forma mais acentuada do que a do modelo tradicional, onde esta relação inversa já existia, porém de modo independente da distribuição de riqueza).

Na equação que relaciona $r_{M}-r_{f} \operatorname{com} \theta_{M}$, nós temos que somente $r_{M}$ e $\theta_{M}$ são endógenos, mais ainda, se $\theta_{M}$ diminui, $r_{M}-r_{f}$ aumenta, o que somente pode ocorrer se $r_{M}$ aumenta. Nós temos uma previsão que um pequeno $\theta_{M}$ (menor que o tradicional) leva a um aumento do excesso de retorno do mercado.

No próximo capítulo vamos refazer a construção do CAPM com influência social e analisando suas consequências, porém tomando hipóteses mais relaxadas com relação a distribuição probabilística das crenças heterogêneas e fazendo uso da utilidade quadrática. Vamos fazer isto a fim de verificar e fortalecer as previsões empíricas e os resultados aqui obtidos. 


\title{
5 Modelo CAPM Uniperiódico com Influên- cia Social - Utilidade Quadrática
}

\begin{abstract}
The attraction of the CAPM is its powerfully simple logic and intuitively pleasing predictions about how to measure risk and about the relation between expected return and risk. Unfortunately, perhaps because of its simplicity, the empirical record of the model is poor - poor enough to invalidate the way it is used in applications... The CAPM, like Markowitz's portfolio model on which it is built, is...a theoretical tour de force. We continue to teach the CAPM as an introduction to the fundamentals of portfolio theory and asset pricing...but we also warn students that, despite its seductive simplicity, the CAPM's empirical problems probably invalidate its use in applications...
\end{abstract}

Eugene Fama (Chicago) - Kenneth French (Dartmouth)

The Capital Asset Pricing Model: Theory and Evidence

Na citação que abre este capítulo é feita uma crítica ao CAPM, em parte esta crítica está relacionada as aplicações do modelo. Muito bem, vamos neste capítulo refazer o nosso CAPM com influência social, com hipóteses mais relaxadas sobre a influência social e mostrar que ainda assim, o modelo leva a previsões que podem ser testadas e são de interesse prático.

\subsection{Modelo do Mercado}

Neste capítulo apresentamos, novamente, um modelo de apreçamento de ativos comportamental baseado em influência social. Desta vez, vamos utilizar a utilidade quadrática. Vamos inicialmente detalhar as hipóteses do modelo:

Consideremos o caso uniperiódico: vamos supor que nosso mercado é formado por $m$ agentes e $n+1$ ativos, sendo $n$ ativos com risco (ou seja, com retorno estocástico) e 1 ativo livre de risco. Dado um agente $a$, sua alocação na carteira de ativos com risco, ou simplesmente sua alocação, é dada por

$$
\vec{X}_{a}=\left(x_{1 a}, \ldots, x_{n a}\right) \in \mathbb{R}^{n}
$$


A alocação no ativo livre é dada por $x_{0 a}=1-\sum_{k=1}^{n} x_{k a}$.

Novamente, vamos supor que não há custos de transação para os investidores. A estrutura probabilística dos retornos dos ativos arriscados é dada por uma gaussiana multidimensional ( $\pi$ com dimensão $n$ ) com vetor de médias $\vec{\mu}_{1}=\left(r_{1}, \ldots, r_{n}\right)$ e matriz de covariâncias $\Sigma$. O retorno $R_{i}$ de um ativo $i$ é definido como

$$
R_{i}=\frac{S_{f}-S_{i}}{S_{i}}
$$

onde $S_{i}$ é o valor do ativo no instante inicial e $S_{f}$ é o valor do ativo no instante final considerados.

A riqueza de um agente no instante final é dada por:

$$
W_{a}\left(\vec{X}_{a}\right)=W_{a}^{0}\left[1+r_{f}+\sum_{k=1}^{n} x_{k a}\left(R_{k}-r_{f}\right)\right]
$$

onde $W_{a}^{0}$ é a riqueza no instante inicial e $r_{f}$ é retorno do ativo livre de risco (taxa de juros).

Em nosso modelo, a utilidade depende não apenas da riqueza do próprio agente, mas também da diferença média entre a riqueza do agente $a$ e dos demais agentes (aqui estamos capturando a influência social dos demais agentes na utilidade percebida pelo agente $a$ ). Esta influência é feita de modo perturbativo, como vamos utilizar a função utilidade quadrática generalizada, temos dois parâmetros de controle $\lambda_{1}$ e $\lambda_{2}$, onde $0<\lambda_{1}<<c$ e $0<\lambda_{2}<<b$ que representam perturbações respectivamente em $c$ e $b$.

Para simplificar a notação, estamos adotando $c_{a}=c$ e $b_{a}=b$, porém em princípio, estes parâmetros poderiam depender do agente $a$, (como o parâmetro $\theta$ no capítulo anterior), mais a frente, quando formos analisar algumas consequências e aplicar a Lei dos Grandes Números, vamos considerar estes parâmetros como sendo os mesmos para todos os agentes do mercado.

Assim, a utilidade do agente $a$ é dada por:

$$
U\left(W_{a}, W_{-a}\right)=\left(c+\lambda_{1}\right) W_{a}-\lambda_{1} \bar{W}_{-a}-\frac{\left(b+\lambda_{2}\right)}{2}\left(W_{a}\right)^{2}-\frac{\lambda_{2}}{2} \overline{W_{-a}^{2}}
$$


Outra hipótese bastante importante de nosso modelo, é a crença heterogênea dos agentes em relação à alocação dos demais, isso é modelado da seguinte forma: o investidor $a$ acredita numa certa distribuição de probabilidades para a alocação dos demais agentes para cada ativo $i$.

A estrutura probabilística das crenças de $a$ quanto à alocação do ativo $i$ pelos demais investidores é uma distribuição multidimensional $\left(\pi_{a i}\right.$ com dimensão $m-1)$ com vetor de médias $\left(x_{i a}, \ldots, x_{i a}\right)=x_{i a} .1$, onde 1 é o vetor com uns em todas as entradas e com a dimensão apropriada, e matriz de covariâncias $\Xi_{a}^{i}$, observe que a estrutura depende de cada investidor e de cada ativo. Note que não há necessidade de crenças em relação à alocação no ativo livre de risco.

A distribuição completa das crenças heterogêneas de $a, \pi_{a}$, é o produto direto das distribuições $\pi_{a i}$, ou seja:

$$
\pi_{a}=\bigotimes \pi_{a i}, 1 \leq i \leq n
$$

tendo vetor de médias $\left(x_{1 a}, \ldots, x_{1 a}, x_{2 a}, \ldots, x_{2 a}, \ldots, x_{n a}, \ldots, x_{n a}\right)$, com dimensão $n(m-1)$ e matriz de covariâncias

$$
\Xi_{a}=\left[\begin{array}{ccccc}
\Xi_{a}^{1} & \mathbb{O} & \ldots & \ldots & \mathbb{O} \\
\mathbb{O} & \ddots & \mathbb{O} & \ldots & \vdots \\
\vdots & \vdots & \Xi_{a}^{i} & \mathbb{O} & \vdots \\
\vdots & \vdots & \mathbb{O} & \ddots & \mathbb{O} \\
\mathbb{O} & \ldots & \ldots & \mathbb{O} & \Xi_{a}^{n}
\end{array}\right]
$$

de dimensão $(n(m-1))^{2}=n^{2}(m-1)^{2}$.

Note que aqui há uma simplificação, por exemplo, dados dois agentes $g$ e $h$ e os ativos $j$ e $k$, a covariância entre a crença do agente $a$ quanto a decisão do agente $g$ relativa ao ativo $j$ e a crença de $a$ quanto a decisão do agente $h$ relativa ao ativo $k$ é nula. Em outras palavras, a covariância entre ativos diferentes para agentes diferentes é nula.

Um ponto a ser enfatizado é que as crenças do agente $a$ são sobre as alocações dos demais agentes, ou seja, sobre as ações que eles vão realizar e NÃO sobre 
suas riquezas no instante inicial.

Aqui temos uma diferença fundamental, como na utilidade quadrática somente aparecem termos ligados aos dois primeiros momentos (média e variância), não precisamos fazer hipóteses sobre toda a distribuição probabilística das crenças, é suficiente um conjunto de hipóteses sobre o vetor de médias e sobre a matriz de covariâncias. Este é um dos motivos de utilizar a utilidade quadrática, além de permitir obter soluções fechadas, temos um relaxamento sobre como se dá a influência social, o que aumenta a generalidade das conclusões. Podemos ver isto como se não fosse necessário uma crença completa a respeito do "véu", basta, para cada agente $a$, hipóteses sobre os dois primeiros momentos do "véu".

O problema do agente $a$ é

$$
\max _{\vec{X}_{a}}\left\{\mathbb{E}_{R} \mathbb{E}_{\pi_{a}}\left[U\left(W_{a}, W_{-a}\right)\right]\right\}
$$

Observe novamente que em relação a situação tradicional (sem influência social) o que temos agora, como argumento da esperança em relação à medida $\pi$ é uma esperança da utilidade com relação à crença sobre os outros agentes, isto pode ser visto como um "véu" que impede $a$ de saber exatamente como os demais agentes irão realizar suas alocações, o que por sua vez influencia na alocação de $a$.

Para calcular o termo $\mathbb{E}_{R} \mathbb{E}_{\pi_{a}}\left[U\left(W_{a}, W_{-a}\right)\right]$, devemos realizar algumas manipulações. Vamos inicialmente começar com a função de utilidade quadrática efetiva $\left(U_{e}\left(V_{a}\right)\right)$ :

$\mathbb{E}_{\pi_{a}}\left[U\left(V_{a}, V_{-a}\right)\right]=\left(c+\lambda_{1}\right) W_{a}-\frac{\left(b+\lambda_{2}\right)}{2}\left(W_{a}\right)^{2}-\lambda_{1} \mathbb{E}_{\pi_{a}}\left[\bar{W}_{-a}\right]+\frac{\lambda_{2}}{2} \mathbb{E}_{\pi_{a}}\left[\overline{W_{-a}^{2}}\right]$

Devemos calcular os termos $\mathbb{E}_{\pi_{a}}\left[\bar{W}_{-a}\right]$ e $\mathbb{E}_{\pi_{a}}\left[\overline{W_{-a}^{2}}\right]$, conforme as hipóteses adotadas no nosso modelo sobre as crenças de $a$.

Temos

$$
\bar{W}_{-a}=\frac{1}{m-1} \sum_{j=1 j \neq a}^{m} W_{j}^{0}\left(1+r_{f}+\sum_{k=1}^{n} x_{k j}\left(R_{k}-r_{f}\right)\right)
$$


Assim,

$$
\begin{aligned}
\mathbb{E}_{\pi_{a}}\left[\bar{W}_{-a}\right] & =\frac{1}{m-1} \sum_{j=1 j \neq a}^{m} W_{j}^{0}\left(1+r_{f}+\sum_{k=1}^{n} \mathbb{E}_{\pi_{a}}\left[x_{k j}\right]\left(R_{k}-r_{f}\right)\right) \\
& =\frac{1}{m-1} \sum_{j=1 j \neq a}^{m} W_{j}^{0}\left(1+r_{f}+\sum_{k=1}^{n} x_{k a}\left(R_{k}-r_{f}\right)\right) \\
& =1+r_{f}+\sum_{k=1}^{n} x_{k a}\left(R_{k}-r_{f}\right)\left(\frac{\sum_{j=1 j \neq a}^{m} W_{j}^{0}}{m-1}\right) \\
& =W_{-a}^{0}\left(1+r_{f}+\sum_{k=1}^{n} x_{k a}\left(R_{k}-r_{f}\right)\right)
\end{aligned}
$$

Onde

$$
\bar{W}_{-a}^{0}=\frac{\sum_{j=1 j \neq a}^{m} W_{j}^{0}}{m-1}
$$

representa a riqueza média dos demais agentes diferentes de $a$ no instante inicial.

Da identidade $\mathbb{E}\left[X^{2}\right]=\mathbb{V} \mathbb{R}[X]+(\mathbb{E}[X])^{2}$ temos que:

$$
\begin{aligned}
\mathbb{E}_{\pi_{a}}\left[\overline{W_{-a}^{2}}\right] & =\mathbb{E}_{\pi_{a}}\left[\frac{\sum_{j=1 j \neq a}^{m} W_{j}^{2}}{m-1}\right] \\
& =\frac{1}{m-1} \sum_{j=1 j \neq a}^{m} \mathbb{E}_{\pi_{a}}\left[W_{j}^{2}\right] \\
& =\frac{1}{m-1} \sum_{j=1 j \neq a}^{m}\left(\mathbb{E}_{\pi_{a}}\left[W_{j}\right]\right)^{2}+\frac{1}{m-1} \sum_{j=1 j \neq a}^{m} \mathbb{V} \mathbb{R} \mathbb{R}_{\pi_{a}}\left[W_{j}\right]
\end{aligned}
$$

Vamos calcular o primeiro termo: 


$$
\begin{aligned}
& \frac{1}{m-1} \sum_{j=1 j \neq a}^{m}\left(\mathbb{E}_{\pi_{a}}\left[W_{j}\right]\right)^{2}= \\
& \frac{1}{m-1} \sum_{j=1 j \neq a}^{m}\left(1+r_{f}+\sum_{k=1}^{n} x_{k a}\left(R_{k}-r_{f}\right)\right)^{2} \\
= & \left(1+r_{f}+\sum_{k=1}^{n} x_{k a}\left(R_{k}-r_{f}\right)\right)^{2}\left(\frac{\sum_{j=1 j \neq a}^{m}\left(W_{j}^{0}\right)^{2}}{m-1}\right) \\
= & \left(1+r_{f}+\sum_{k=1}^{n} x_{k a}\left(R_{k}-r_{f}\right)\right)^{2} \overline{\left(W_{-a}^{0}\right)^{2}} \\
= & \left(1+r_{f}+\left(\vec{R}-r_{f} \mathbf{1}\right) \cdot \vec{X}_{a}\right)^{2} \frac{\left(W_{-a}^{0}\right)^{2}}{}
\end{aligned}
$$

Onde

$$
\overline{\left(W_{-a}^{0}\right)^{2}}=\frac{\sum_{j=1 j \neq a}^{m}\left(W_{j}^{0}\right)^{2}}{m-1}
$$

representa a média das riquezas ao quadrado dos demais agentes diferentes de $a$ no instante inicial.

$$
\begin{aligned}
\mathbb{V} \mathbb{R}_{\pi_{a}}\left[W_{j}\right]= & \mathbb{V} \mathbb{R}_{\pi_{a}}\left[W_{j}^{0}\left(1+r_{f}+\left(\vec{R}-r_{f} \mathbf{1}\right)\right)\right] \\
= & \left(W_{j}^{0}\right)^{2}\left(\sum_{k=1}^{n} \mathbb{V} \mathbb{R}_{\pi_{a}}\left[x_{k a}\right]\left(R_{k}-r_{f}\right)^{2}\right) \\
& +2\left(W_{j}^{0}\right)^{2}\left(\sum_{l, p=1, l<p}^{n} \operatorname{cov}_{\pi_{a}}\left[x_{l a}, x_{p a}\right]\left(R_{l}-r_{f}\right)\left(R_{p}-r_{f}\right)\right) \\
= & \left.\left(W_{j}^{0}\right)^{2}\left(\sum_{k=1}^{n}\left(\Xi_{a}^{k}\right)_{j j}\left(R_{k}-r_{f}\right)^{2}\right)\right)
\end{aligned}
$$

Note que os termos $\operatorname{cov}_{\pi_{a}}\left[x_{l a}, x_{p a}\right]$ são nulos devido a nossa hipótese sobre o 
formato da distribuição $\pi_{a}$ das crenças heterogêneas.

Considere as matrizes elementares $E_{i j} \operatorname{com}\left(E_{i j}\right)=\delta_{i j}$ onde $\delta_{i j}$ é o delta de Kronecker, podemos reescrever o termo $\left.\sum_{k=1}^{n}\left(\Xi_{a}^{k}\right)_{j j}\left(R_{k}-r_{f}\right)^{2}\right)$ como $\left(\vec{R}-r_{f} \mathbf{1}\right) \mathbb{W}_{j}\left(\vec{R}-r_{f} \mathbf{1}\right)$ onde $\mathbb{W}_{j}=\sum_{k=1}^{n}\left(\Xi_{a}^{k}\right)_{j j} E_{k k}$, assim podemos escrever:

$$
\mathbb{V} \mathbb{R}_{\pi_{a}}\left[W_{j}\right]=\left(W_{j}^{0}\right)^{2}\left(\vec{R}-r_{f} \mathbf{1}\right) \cdot \mathbb{W}_{j}\left(\vec{R}-r_{f} \mathbf{1}\right)
$$

Finalmente, vamos calcular o segundo termo:

$$
\frac{1}{m-1} \sum_{j=1 j \neq a}^{m} \mathbb{V} \mathbb{R}_{\pi_{a}}\left[W_{j}\right]=\frac{1}{m-1} \sum_{j=1 j \neq a}^{m}\left(\left(W_{j}^{0}\right)^{2}\left(\vec{R}-r_{f} \mathbf{1}\right) \cdot \mathbb{W}_{j}\left(\vec{R}-r_{f} \mathbf{1}\right)\right)
$$

Agora, podemos prosseguir, juntando os dois termos calculados, temos:

$$
\begin{aligned}
\mathbb{E}_{\pi_{a}}\left[\overline{W_{-a}^{2}}\right]= & \frac{1}{m-1} \sum_{j=1 j \neq a}^{m}\left(\mathbb{E}_{\pi_{a}}\left[W_{j}\right]\right)^{2}+\frac{1}{m-1} \sum_{j=1 j \neq a}^{m} \mathbb{V} \mathbb{R}_{\pi_{a}}\left[W_{j}\right] \\
= & \left(1+r_{f}+\left(\vec{R}-r_{f} \mathbf{1}\right) \cdot \vec{X}_{a}\right)^{2} \overline{\left(W_{-a}^{0}\right)^{2}} \\
& +\frac{1}{m-1} \sum_{j=1 j \neq a}^{m}\left(\left(W_{j}^{0}\right)^{2}\left(\vec{R}-r_{f} \mathbf{1}\right) \cdot \mathbb{W}_{j}\left(\vec{R}-r_{f} \mathbf{1}\right)\right)
\end{aligned}
$$

Vamos retornar ao cálculo da utilidade efetiva: 


$$
\begin{aligned}
& \mathbb{E}_{\pi_{a}}\left[U\left(W_{a}, W_{-a}\right)\right]= \\
& \left(c+\lambda_{1}\right) W_{a}-\frac{\left(b+\lambda_{2}\right)}{2}\left(W_{a}\right)^{2}-\lambda_{1} \mathbb{E}_{\pi_{a}}\left[\bar{W}_{-a}\right]+\frac{\lambda_{2}}{2} \mathbb{E}_{\pi_{a}}\left[\overline{W_{-a}^{2}}\right] \\
& =\left(c+\lambda_{1}\right) W_{a}-\frac{\left(b+\lambda_{2}\right)}{2}\left(W_{a}\right)^{2} \\
& -\lambda_{1}\left(1+r_{f}+\left(\vec{R}-r_{f} \mathbf{1}\right)\right) \bar{W}_{-a}^{0} \\
& +\frac{\lambda_{2}}{2}\left(1+r_{f}+\left(\vec{R}-r_{f} \mathbf{1}\right) \cdot \vec{X}_{a}\right)^{2} \overline{\left(W_{-a}^{0}\right)^{2}} \\
& +\frac{\lambda_{2}}{2} \frac{1}{m-1} \sum_{j=1 j \neq a}^{m}\left(\left(\vec{R}-r_{f} \mathbf{1}\right) \cdot \mathbb{W}_{j}\left(\vec{R}-r_{f} \mathbf{1}\right)\left(W_{j}^{0}\right)^{2}\right) \\
& =\left(c+\lambda_{1}\right) W_{a}^{0}\left(1+r_{f}+\left(\vec{R}-r_{f} \mathbf{1}\right) \cdot \vec{X}_{a}\right) \\
& -\frac{\left(b+\lambda_{2}\right)}{2}\left(W_{a}^{0}\right)^{2}\left(1+r_{f}+\left(\vec{R}-r_{f} \mathbf{1}\right) \cdot \vec{X}_{a}\right)^{2} \\
& -\lambda_{1} \bar{W}_{-a}^{0}\left(1+r_{f}+\left(\vec{R}-r_{f} \mathbf{1}\right)\right) \\
& +\frac{\lambda_{2}}{2} \overline{\left(W_{-a}^{0}\right)^{2}}\left(1+r_{f}+\left(\vec{R}-r_{f} \mathbf{1}\right) \cdot \vec{X}_{a}\right)^{2} \\
& +\frac{\lambda_{2}}{2} \frac{1}{m-1} \sum_{j=1 j \neq a}^{m}\left(\left(\vec{R}-r_{f} \mathbf{1}\right) \cdot \mathbb{W}_{j}\left(\vec{R}-r_{f} \mathbf{1}\right)\left(W_{j}^{0}\right)^{2}\right) \\
& =\left(c W_{a}^{0}+\lambda_{1}\left(W_{a}^{0}-\bar{W}_{-a}^{0}\right)\right)\left(1+r_{f}+\left(\vec{R}-r_{f} \mathbf{1}\right) \cdot \vec{X}_{a}\right) \\
& -\frac{1}{2}\left(b\left(W_{a}^{0}\right)^{2}+\lambda_{2}\left(\left(W_{a}^{0}\right)^{2}-\overline{\left(W_{-a}^{0}\right)^{2}}\right)\right)\left(1+r_{f}+\left(\vec{R}-r_{f} \mathbf{1}\right) \cdot \vec{X}_{a}\right)^{2} \\
& +\frac{\lambda_{2}}{2} \frac{1}{m-1} \sum_{j=1 j \neq a}^{m}\left(\left(\vec{R}-r_{f} \mathbf{1}\right) \cdot \mathbb{W}_{j}\left(\vec{R}-r_{f} \mathbf{1}\right)\left(W_{j}^{0}\right)^{2}\right)
\end{aligned}
$$

Vamos proceder agora ao cálculo da esperança segundo a distribuição dos ativos, para isso vamos calcular em cada um dos três termos nos quais separamos a utilidade efetiva $\mathbb{E}_{\pi_{a}}\left[U\left(W_{a}, W_{-a}\right)\right]$ :

Para o primeiro termo temos:

$$
\begin{aligned}
& \mathbb{E}_{R}\left[\left(c W_{a}^{0}+\lambda_{1}\left(W_{a}^{0}-\bar{W}_{-a}^{0}\right)\right)\left(1+r_{f}+\left(\vec{R}-r_{f} \mathbf{1}\right) \cdot \vec{X}_{a}\right)\right]= \\
& \left(c W_{a}^{0}+\lambda_{1}\left(W_{a}^{0}-\bar{W}_{-a}^{0}\right)\right)\left(1+r_{f}+\left(\vec{\mu}_{1}-r_{f} \mathbf{1}\right) \cdot \vec{X}_{a}\right)
\end{aligned}
$$


Para o segundo termo temos:

$$
\begin{aligned}
& \mathbb{E}_{R}\left[-\frac{1}{2}\left(b\left(W_{a}^{0}\right)^{2}+\lambda_{2}\left(\left(W_{a}^{0}\right)^{2}-\overline{\left(W_{-a}^{0}\right)^{2}}\right)\right)\left(1+r_{f}+\left(\vec{R}-r_{f} \mathbf{1}\right) \cdot \vec{X}_{a}\right)^{2}\right] \\
= & -\frac{1}{2}\left(b\left(W_{a}^{0}\right)^{2}+\lambda_{2}\left(\left(W_{a}^{0}\right)^{2}-\overline{\left(W_{-a}^{0}\right)^{2}}\right)\right) \\
\times & \mathbb{E}_{R}\left[\left(1+r_{f}\right)^{2}+2\left(1+r_{f}\right)\left(\vec{R}-r_{f} \mathbf{1}\right) \cdot \vec{X}_{a}+\left(\left(\vec{R}-r_{f} \mathbf{1}\right) \cdot \vec{X}_{a}\right)^{2}\right]
\end{aligned}
$$

Vamos calcular inicialmente a esperança do termo quadrático $\left(\left(\vec{R}-r_{f} \mathbf{1}\right) \cdot \vec{X}_{a}\right)^{2}$. Seja a matriz $\mathbb{D}$ onde cada elemento $d_{j k}$ é dado por $d_{j k}=\left(r_{j}-r_{f}\right)\left(r_{k}-r_{f}\right)$ onde $r_{j}$ e $r_{k}$ são esperanças dos retornos dos ativos $j$ e $k$ e $r_{f}$ é o retorno do ativo livre de risco. Definimos a matriz $\mathbb{M}$ como sendo:

$$
\mathbb{M}=\Sigma+\mathbb{D}
$$

onde $\Sigma$ é a matriz de covariâncias dos retornos dos ativos. Como $\Sigma$ e $\mathbb{D}$ são simétricas e $\Sigma$ tem inversa por hipótese, temos que $\mathbb{M}$ é simétrica e tem inversa. Assim, temos:

$$
\begin{aligned}
& \mathbb{E}_{R}\left[\left(\left(\vec{R}-r_{f} \mathbf{1}\right) \cdot \vec{X}_{a}\right)^{2}\right]= \\
& \mathbb{E}_{R}\left[\sum_{i=1}^{n}\left(\left(R_{i}-r_{f}\right)^{2} x_{a i}^{2}\right)+2 \sum_{j, k=1, j<k}^{n}\left(\left(R_{j}-r_{f}\right)\left(R_{k}-r_{f}\right) x_{a j} x_{a k}\right)\right] \\
= & \sum_{i=1}^{n} \mathbb{E}_{R}\left[\left(R_{i}-r_{f}\right)^{2} x_{a i}^{2}\right]+2 \sum_{j, k=1, j<k}^{n} \mathbb{E}_{R}\left[\left(R_{j}-r_{f}\right)\left(R_{k}-r_{f}\right) x_{a j} x_{a k}\right] \\
= & \left.\sum_{i=1}^{n}\left(\left(\left(r_{i}-r_{f}\right)^{2}+\Sigma_{i i}\right) x_{a i}^{2}\right)+2 \sum_{j, k=1, j<k}^{n}\left(\left(r_{j}-r_{f}\right)\left(r_{k}-r_{f}\right)+\Sigma_{j k}\right) x_{a j} x_{a k}\right) \\
= & \vec{X}_{a} \Sigma \cdot \vec{X}_{a}+\vec{X}_{a} \mathbb{D} \cdot \vec{X}_{a} \\
= & \vec{X}_{a} \mathbb{M} \cdot \vec{X}_{a}
\end{aligned}
$$


Agora, podemos retornar ao cálculo do segundo termo:

$$
\begin{aligned}
& \mathbb{E}_{R}\left[-\frac{1}{2}\left(b\left(W_{a}^{0}\right)^{2}+\lambda_{2}\left(\left(W_{a}^{0}\right)^{2}-\overline{\left(W_{-a}^{0}\right)^{2}}\right)\right)\left(1+r_{f}+\left(\vec{R}-r_{f} \mathbf{1}\right) \cdot \vec{X}_{a}\right)^{2}\right] \\
= & -\frac{1}{2}\left(b\left(W_{a}^{0}\right)^{2}+\lambda_{2}\left(\left(W_{a}^{0}\right)^{2}-\overline{\left(W_{-a}^{0}\right)^{2}}\right)\right) \\
\times & \left(\left(1+r_{f}\right)^{2}+2\left(1+r_{f}\right)\left(\vec{\mu}_{1}-r_{f} \mathbf{1}\right) \cdot \vec{X}_{a}+\vec{X}_{a} \mathbb{M} \cdot \vec{X}_{a}\right)
\end{aligned}
$$

Agora, observe o terceiro termo, como ele não depende de $\vec{X}_{a}$, quando efetuarmos o próximo passo que consiste em calcular o gradiente de com relação a $\vec{X}_{a}$, temos que este termo desaparecerá, assim não precisamos nos preocupar em calcular a esperança dele em relação a distribuição do retorno dos ativos. Este fato é há algo surprendente pois significa que as crenças heterogêneas sobre as distribuições dos demais agentes do mercado só têm impacto no primeiro momento (esperança) sobre o agente $a$, isto já ocorreu antes, no capítulo anterior, com a função de utilidade exponencial generalizada.

Para simplificar a notação vamos fazer $\alpha=\left(c W_{a}^{0}+\lambda_{1}\left(W_{a}^{0}-\bar{W}_{-a}^{0}\right)\right)$ e $\beta=$ $\left(b\left(W_{a}^{0}\right)^{2}+\lambda_{2}\left(\left(W_{a}^{0}\right)^{2}-\overline{\left(W_{-a}^{0}\right)^{2}}\right)\right)$, note que $\alpha$ e $\beta$ são como coeficientes generalizados da utilidade quadrática (multiplicados pelas riquezas iniciais) e que ambos levam em consideração o efeito da influência social, assim ambos dependem do agente $a$, bem como dos demais agentes do mercado. Temos então: 


$$
\begin{aligned}
& \mathbb{E}_{R} \mathbb{E}_{\pi_{a}}\left[U\left(W_{a}, W_{-a}\right)\right]= \\
& \left(c W_{a}^{0}+\lambda_{1}\left(W_{a}^{0}-\bar{W}_{-a}^{0}\right)\right)\left(1+r_{f}+\left(\vec{\mu}_{1}-r_{f} \mathbf{1}\right) \cdot \vec{X}_{a}\right) \\
- & \frac{1}{2}\left(b\left(W_{a}^{0}\right)^{2}+\lambda_{2}\left(\left(W_{a}^{0}\right)^{2}-\overline{\left(W_{-a}^{0}\right)^{2}}\right)\right) \\
& \times\left(\left(1+r_{f}\right)^{2}+2\left(1+r_{f}\right)\left(\vec{\mu}_{1}-r_{f} \mathbf{1}\right) \cdot \vec{X}_{a}+\vec{X}_{a} \mathbb{M} \cdot \vec{X}_{a}\right) \\
+ & \mathbb{E}_{R}\left[\frac{\lambda_{2}}{2} \frac{1}{m-1} \sum_{j=1 j \neq a}^{m}\left(\left(\vec{R}-r_{f} \mathbf{1}\right) \cdot \mathbb{W}_{j}\left(\vec{R}-r_{f} \mathbf{1}\right)\left(W_{j}^{0}\right)^{2}\right)\right] \\
= & \alpha\left(1+r_{f}+\left(\vec{\mu}_{1}-r_{f} \mathbf{1}\right) \cdot \vec{X}_{a}\right) \\
- & \frac{1}{2} \beta\left(\left(1+r_{f}\right)^{2}+2\left(1+r_{f}\right)\left(\vec{\mu}_{1}-r_{f} \mathbf{1}\right) \cdot \vec{X}_{a}+\vec{X}_{a} \mathbb{M} \cdot \vec{X}_{a}\right) \\
+ & \mathbb{E}_{R}\left[\frac{\lambda_{2}}{2} \frac{1}{m-1} \sum_{j=1 j \neq a}^{m}\left(\left(\vec{R}-r_{f} \mathbf{1}\right) \cdot \mathbb{W}_{j}\left(\vec{R}-r_{f} \mathbf{1}\right)\left(W_{j}^{0}\right)^{2}\right)\right]
\end{aligned}
$$

Vamos calcular o gradiente da esperança da função utilidade, assim temos:

$$
\begin{aligned}
\frac{\partial}{\partial \vec{X}_{a}}\left(\mathbb{E}_{R} \mathbb{E}_{\pi_{a}}\left[U\left(W_{a}, W_{-a}\right)\right]\right)= & \alpha\left(1+r_{f}+\left(\vec{\mu}_{1}-r_{f} \mathbf{1}\right)\right) \\
& -\frac{1}{2} \beta\left(2\left(1+r_{f}\right)\left(\vec{\mu}_{1}-r_{f} \mathbf{1}\right)+\mathbb{M} \vec{X}_{a}\right) \\
= & \left(\alpha-\beta\left(1+r_{f}\right)\right)\left(\vec{\mu}_{1}-r_{f} \mathbf{1}\right)-\beta \mathbb{M} \vec{X}_{a}
\end{aligned}
$$

No equilíbrio devemos ter o gradiente nulo (condições de primeira ordem):

$$
\begin{aligned}
\frac{\partial}{\partial \vec{X}_{a}}\left(\mathbb{E}_{R} \mathbb{E}_{\pi_{a}}\left[U\left(W_{a}, W_{-a}\right)\right]\right) & =\overrightarrow{0} \Leftrightarrow \\
\left(\alpha-\beta\left(1+r_{f}\right)\right)\left(\vec{\mu}_{1}-r_{f} \mathbf{1}\right)-\beta \mathbb{M} \vec{X}_{a}^{*} & =\overrightarrow{0}
\end{aligned}
$$




$$
\begin{aligned}
\Leftrightarrow \vec{X}_{a}^{*} & =\left(\frac{\alpha}{\beta}-\left(1+r_{f}\right)\right) \mathbb{M}^{-1}\left(\vec{r}_{e}\right) \\
\vec{X}_{a}^{*} & =\left(\frac{\left(c W_{a}^{0}+\lambda_{1}\left(W_{a}^{0}-\bar{W}_{-a}^{0}\right)\right)}{\left(b\left(W_{a}^{0}\right)^{2}+\lambda_{2}\left(\left(W_{a}^{0}\right)^{2}-\overline{\left(W_{-a}^{0}\right)^{2}}\right)\right)}-\left(1+r_{f}\right)\right) \mathbb{M}^{-1}\left(\vec{\mu}_{1}-r_{f} \mathbf{1}\right)
\end{aligned}
$$

Em particular, (omitindo somatórios quando há índices repetidos)

$$
x_{a i}^{*}=\left(\frac{\alpha}{\beta}-\left(1+r_{f}\right)\right) M_{k i}^{-1}\left(r_{k}-r_{f}\right)
$$

Por outro lado,

$$
\begin{gathered}
\vec{X}_{a}^{*}=\left(\frac{\alpha}{\beta}-\left(1+r_{f}\right)\right) \mathbb{M}^{-1}\left(\vec{r}_{e}\right) \Leftrightarrow \\
\vec{r}_{e}=\left(\frac{\alpha}{\beta}-\left(1+r_{f}\right)\right)^{-1} \vec{X}_{a}^{*} \mathbb{M}
\end{gathered}
$$

Em particular, (omitindo somatórios quando há índices repetidos)

$$
\begin{aligned}
r_{i}-r_{f} & =\left(\frac{\alpha}{\beta}-\left(1+r_{f}\right)\right)^{-1} x_{a l}^{*} M_{l} i \\
& =\left(\frac{\alpha}{\beta}-\left(1+r_{f}\right)\right)^{-1}\left(\frac{\alpha}{\beta}-\left(1+r_{f}\right)\right) M_{k l}^{-1}\left(r_{k}-r_{f}\right) M_{l i} \\
& =\left(r_{k}-r_{f}\right) M_{k l}^{-1} M_{l i}
\end{aligned}
$$




\subsection{CAPM com Influência Social}

Vamos agora, em busca de uma relação entre os retornos esperados dos ativos e o do mercado como no CAPM tradicional, só que agora em nosso modelo.

Por definição, temos $W_{0}^{M}=\sum_{i=1}^{n} W_{a}^{0}$. Considere a seguinte identidade:

$$
\begin{aligned}
x_{a i}^{*} & =\left(\frac{\alpha}{\beta}-\left(1+r_{f}\right)\right) M_{k i}^{-1}\left(r_{k}-r_{f}\right) \Rightarrow \\
\sum_{i=1}^{n} \frac{W_{a}^{0}}{W_{M}^{0}} x_{a i}^{*}\left(r_{i}-r_{f}\right) & =\sum_{i=1}^{n} \frac{W_{a}^{0}\left(\frac{\alpha}{\beta}-\left(1+r_{f}\right)\right)\left(r_{k}-r_{f}\right) M_{k i}^{-1}\left(r_{i}-r_{f}\right)}{W_{M}^{0}} \\
& =\frac{W_{a}^{0}\left(\frac{\alpha}{\beta}-\left(1+r_{f}\right)\right) \vec{r}_{e} \mathbb{M}^{-1} \vec{r}_{e}}{W_{M}^{0}}
\end{aligned}
$$

Somando esta identidade para todos os agentes do mercado obtemos:

$$
\begin{aligned}
r_{M}-r_{f}= & \frac{\vec{r}_{e} \mathbb{M}^{-1} \vec{r}_{e}}{W_{M}^{0}} \sum_{a=1}^{m} W_{a}^{0}\left(\frac{\alpha}{\beta}-\left(1+r_{f}\right)\right) \\
& \frac{\vec{r}_{e} \mathbb{M}^{-1} \vec{r}_{e}}{W_{M}^{0}}\left(\sum_{a=1}^{m} W_{a}^{0} \frac{\alpha}{\beta}\right)-\frac{\vec{r}_{e} \mathbb{M}^{-1} \vec{r}_{e}\left(1+r_{f}\right)}{W_{M}^{0}} \sum_{a=1}^{m} W_{a}^{0} \\
= & \frac{\vec{r}_{e} \mathbb{M}^{-1} \vec{r}_{e}}{W_{M}^{0}}\left(\sum_{a=1}^{m} W_{a}^{0} \frac{\alpha}{\beta}\right)-\vec{r}_{e} \mathbb{M}^{-1} \vec{r}_{e}\left(1+r_{f}\right)
\end{aligned}
$$

Vamos olhar mais detidamente o seguinte termo: 


$$
\begin{aligned}
\sum_{a=1}^{m} W_{a}^{0} \frac{\alpha}{\beta} & =\sum_{a=1}^{m} W_{a}^{0} \frac{\left(c+\lambda_{1}\right) W_{a}^{0}-\lambda_{1} \bar{W}_{-a}^{0}}{\left(b+\lambda_{2}\right)\left(W_{a}^{0}\right)^{2}-\lambda_{2} \overline{\left(W_{-a}^{0}\right)^{2}}} \\
& =\sum_{a=1}^{m} \frac{\left(c+\lambda_{1}\right)-\lambda_{1} \frac{\bar{W}_{-a}^{0}}{W_{a}^{0}}}{\left(b+\lambda_{2}\right)-\lambda_{2} \frac{\overline{\left(W_{-a}^{0}\right)^{2}}}{\left(W_{a}^{0}\right)^{2}}} \\
& =\sum_{a=1}^{m} \frac{c+\lambda_{1}\left(1-\frac{\bar{W}_{-a}^{0}}{W_{a}^{0}}\right)}{b+\lambda_{2}\left(1-\frac{\overline{\left(W_{-a}^{0}\right)^{2}}}{\left(W_{a}^{0}\right)^{2}}\right)}
\end{aligned}
$$

Comparando com o CAPM tradicional, é razoável denominar este termo como o inverso da aversão ao risco do mercado efetiva e denotá-lo por $\frac{1}{\theta_{M}}$. Observe que se fizermos $\lambda_{1}=\lambda_{2}=0$ o que corresponde a ausência de influência social, recuperamos o caso tradicional. Assim,

$$
r_{M}-r_{f}=\frac{\vec{r}_{e} \mathbb{M}^{-1} \vec{r}_{e}}{W_{M}^{0} \theta_{M}}-\vec{r}_{e} \mathbb{M}^{-1} \vec{r}_{e}\left(1+r_{f}\right)
$$

No equilíbrio, temos

$$
R_{M}=\sum_{a=1}^{m}\left[\frac{W_{a}^{0}}{W_{M}^{0}}\left(r_{f}+\sum_{j=1}^{n} x_{a j}^{*}\left(R_{j}-r_{f}\right)\right)\right]
$$

$\log \mathrm{O}$ 


$$
\begin{aligned}
\operatorname{cov}_{R}\left[R_{i}, R_{M}\right] & =\operatorname{cov}_{R}\left[R_{i}, \sum_{a=1}^{m}\left[\frac{W_{a}^{0}}{W_{M}^{0}}\left(r_{f}+\sum_{j=1}^{n} x_{a j}^{*}\left(R_{j}-r_{f}\right)\right)\right]\right] \\
& =\sum_{a=1}^{m}\left(\frac{W_{a}^{0}}{W_{M}^{0}}\left[\sum_{j=1}^{n} x_{a j}^{*} \operatorname{cov}_{R}\left[R_{i}, R_{j}\right]\right]\right) \\
& =\sum_{a=1}^{m}\left(\frac{W_{a}^{0}}{W_{M}^{0}}\left[\sum_{j=1}^{n} x_{a j}^{*} \Sigma_{i j}\right]\right) \\
& =\sum_{a=1}^{m}\left(\frac{W_{a}^{0}}{W_{M}^{0}}\left[\sum_{j=1}^{n}\left(\frac{\alpha}{\beta}-\left(1+r_{f}\right)\right) M_{k j}^{-1}\left(r_{k}-r_{f}\right) \Sigma_{i j}\right]\right) \\
& =\sum_{a=1}^{m} \frac{W_{a}^{0}\left(\frac{\alpha}{\beta}-\left(1+r_{f}\right)\right)}{W_{M}^{0}}\left(r_{k}-r_{f}\right) M_{k j}^{-1} \Sigma_{i j} \\
& =\left(\frac{\sum_{a=1}^{m} W_{a}^{0} \frac{\alpha}{\beta}}{W_{M}^{0}}-\left(1+r_{f}\right) \frac{\sum_{a=1}^{m} W_{a}^{0}}{W_{M}^{0}}\right)\left(r_{i}-r_{f}\right) \\
& =\left(\frac{1}{\theta_{M} W_{M}^{0}}-\left(1+r_{f}\right)\right)\left(r_{i}-r_{f}\right)
\end{aligned}
$$

Temos também:

$$
\begin{aligned}
& \sigma_{M}^{2}=\operatorname{cov}_{R}\left[R_{M}, R_{M}\right] \\
= & \operatorname{cov}\left[R_{M}, \sum_{a=1}^{m}\left(\frac{W_{a}^{0}}{W_{M}^{0}}\left[r_{f}+\sum_{i=1}^{n} x_{a i}^{*}\left(R_{i}-r_{f}\right)\right]\right)\right] \\
= & \sum_{a=1}^{m}\left(\frac{W_{a}^{0}}{W_{M}^{0}}\left[\sum_{i=1}^{n} x_{a i}^{*} \operatorname{cov}_{R}\left[R_{i}, R_{M}\right]\right]\right) \\
= & \sum_{a=1}^{m}\left(\frac{W_{a}^{0}}{W_{M}^{0}}\left[\left(\frac{\alpha}{\beta}-\left(1+r_{f}\right)\right) M_{k j}^{-1}\left(r_{k}-r_{f}\right)\left(\frac{1}{\theta_{M} W_{M}^{0}}-\left(1+r_{f}\right)\right)\left(r_{i}-r_{f}\right)\right]\right) \\
= & \left(\sum_{a=1}^{m} \frac{W_{a}^{0}}{W_{M}^{0}}\left(\frac{\alpha}{\beta}-\left(1+r_{f}\right)\right)\right)\left(\frac{1}{\theta_{M} W_{M}^{0}}-\left(1+r_{f}\right)\right)\left(\left(r_{k}-r_{f}\right) M_{k i}^{-1}\left(r_{i}-r_{f}\right)\right) \\
= & \left(\frac{1}{\theta_{M} W_{M}^{0}}-\left(1+r_{f}\right)\right)^{2}\left(\vec{r}_{e} \mathbb{M}^{-1} \vec{r}_{e}\right)
\end{aligned}
$$


Fazendo $\gamma=\frac{1}{\theta_{M} W_{M}^{0}}-\left(1+r_{f}\right)$, temos:

$$
\begin{aligned}
\sigma_{M}^{2} & =\gamma^{2} \overrightarrow{r_{e}} \mathbb{M}^{-1} \overrightarrow{r_{e}} \\
\Leftrightarrow \sigma_{M} & =\gamma \sqrt{\overrightarrow{r_{e}} \mathbb{M}^{-1} \overrightarrow{r_{e}}} \\
& =\left(\frac{1}{\theta_{M} W_{M}^{0}}-\left(1+r_{f}\right)\right) \sqrt{\overrightarrow{r_{e}} \mathbb{M}^{-1} \overrightarrow{r_{e}}}
\end{aligned}
$$

onde $\sigma_{M}$ é a volatilidade do mercado e supondo $\gamma>0$, caso contrário devemos tomar o valor absoluto. Assim, podemos observar que a volatilidade do mercado $\left(\sigma_{M}\right)$ varia de maneira inversa à aversão ao risco efetiva do mercado $\left(\theta_{M}\right)$. Isso já ocorria no modelo tradicional, porém aqui, como $\theta_{M}$ está relacionado com a influência social, podemos esperar obter novamente conclusões interessantes, ligando a volatilidade do mercado com influência social.

Agora, vamos relacionar $r_{M}-r_{f} \operatorname{com} r_{i}-r_{f}$ :

Inicialmente observe que:

$$
\begin{aligned}
r_{M}-r_{f} & =\frac{\vec{r}_{e} \mathbb{M}^{-1} \vec{r}_{e}}{W_{M}^{0} \theta_{M}}-\vec{r}_{e} \mathbb{M}^{-1} \vec{r}_{e}\left(1+r_{f}\right) \\
& =\gamma \vec{r}_{e} \mathbb{M}^{-1} \vec{r}_{e}
\end{aligned}
$$

$$
\begin{aligned}
\sigma_{M}^{2}=\operatorname{cov}_{R}\left[R_{M}, R_{M}\right] & =\gamma^{2} \vec{r}_{e} \mathbb{M}^{-1} \vec{r}_{e}=\gamma\left(r_{M}-r_{f}\right) \Leftrightarrow \\
\frac{1}{\gamma} & =\frac{r_{M}-r_{f}}{\operatorname{cov}_{R}\left[R_{M}, R_{M}\right]}
\end{aligned}
$$

Temos também que

$$
\begin{aligned}
\operatorname{cov}_{R}\left[R_{i}, R_{M}\right] & =\gamma\left(r_{i}-r_{f}\right) \Leftrightarrow \\
\frac{1}{\gamma} & =\frac{r_{i}-r_{f}}{\operatorname{cov}_{R}\left[R_{i}, R_{M}\right]}
\end{aligned}
$$


Por outro lado,

$$
\begin{aligned}
\frac{r_{M}-r_{f}}{\operatorname{cov}_{R}\left[R_{M}, R_{M}\right]} & =\frac{r_{i}-r_{f}}{\operatorname{cov}_{R}\left[R_{i}, R_{M}\right]} \Leftrightarrow \\
r_{i}-r_{f} & =\frac{\operatorname{cov}_{R}\left[R_{i}, R_{M}\right]}{\operatorname{cov}_{R}\left[R_{M}, R_{M}\right]}\left(r_{M}-r_{f}\right)
\end{aligned}
$$

Fazendo $\beta_{i}=\frac{\operatorname{cov}_{R}\left[R_{i}, R_{M}\right]}{\operatorname{cov}_{R}\left[R_{M}, R_{M}\right]}$, obtemos o resultado desejado:

Teorema: (CAPM com influência social) O excesso de retorno dos ativos é diretamente proporcional ao excesso de retorno do mercado, onde o coeficiente de proporcionalidade $\beta_{i}$ está relacionado à presença da influência social, algo não presente no caso tradicional.

$$
r_{i}-r_{f}=\beta_{i}\left(r_{M}-r_{f}\right)
$$

Onde,

$$
\begin{aligned}
\beta_{i} & =\frac{\operatorname{cov}_{R}\left[R_{i}, R_{M}\right]}{\operatorname{cov}_{R}\left[R_{M}, R_{M}\right]} \\
& =\frac{1-\left(1+r_{f}\right) W_{M}^{0} \theta_{M}}{W_{M}^{0} \theta_{M}} \frac{\left(r_{i}-r_{f}\right)}{\overrightarrow{r_{e}} \mathbb{M}^{-1} \overrightarrow{r_{e}}}
\end{aligned}
$$

Como vimos anteriormente

$$
\theta_{M}^{-1}=\sum_{a=1}^{m} \frac{c+\lambda_{1}\left(1-\frac{\bar{W}_{-a}^{0}}{W_{a}^{0}}\right)}{b+\lambda_{2}\left(1-\frac{\overline{\left(W_{-a}^{0}\right)^{2}}}{\left(W_{a}^{0}\right)^{2}}\right)}
$$


De modo explícito, em palavras, temos que $\beta_{i}$ depende de $\theta_{M}$ que por sua vez incorpora a presença de influência social o que representa uma novidade em relação a situação tradicional.

\subsection{Analisando Algumas Consequências}

Vamos investigar a influência da aversão ao risco do mercado efetiva em nossa fórmula e comparar com a situação no modelo tradicional. A partir de agora vamos realizar a seguinte simplificação: vamos considerar os termos $c$ e $b$ como sendo constantes (assumem os mesmos valores para todos os agentes $a, 1 \leq a \leq m)$.

\section{Temos:}

$$
\begin{aligned}
\frac{1}{\theta_{M}} & =\sum_{a=1}^{m} \frac{\left(c+\lambda_{1}\right)-\lambda_{1} \frac{\bar{W}_{-a}^{0}}{W_{a}^{0}}}{\left(b+\lambda_{2}\right)-\lambda_{2} \frac{\overline{\left(W_{-a}^{0}\right)^{2}}}{\left(W_{a}^{0}\right)^{2}}} \\
& =\sum_{a=1}^{m} \frac{c+\lambda_{1}\left(1-\frac{\overline{W_{-a}^{0}}}{W_{a}^{0}}\right)}{b+\lambda_{2}\left(1-\frac{\overline{\left(W_{-a}^{0}\right)^{2}}}{\left(W_{a}^{0}\right)^{2}}\right)}
\end{aligned}
$$

Para $m \rightarrow \infty$, pela Lei dos Grandes Números, temos: 


$$
\begin{aligned}
\frac{1}{\theta_{M}} & =\frac{\sum_{a=1}^{m} \frac{c+\lambda_{1}\left(1-\frac{\bar{W}_{-a}^{0}}{W_{a}^{0}}\right)}{b+\lambda_{2}\left(1-\frac{\overline{\left(W_{-a}^{0}\right)^{2}}}{\left(W_{a}^{0}\right)^{2}}\right)}}{m} \Rightarrow \\
\frac{1}{\theta_{m}} & =\int_{\mathbb{R}} \frac{c+\lambda_{1}\left(1-\frac{\mathbb{E}_{W}[W]}{W}\right)}{b+\lambda_{2}\left(1-\frac{\mathbb{E}_{W}\left[W^{2}\right]}{W^{2}}\right)} f(W) d W \Leftrightarrow \\
\frac{1}{\theta_{m}} & =\int_{\mathbb{R}} \frac{\left(c+\lambda_{1}\right)-\lambda_{1}\left(\frac{\mathbb{E}_{W}[W]}{W}\right)}{\left(b+\lambda_{2}\right)-\lambda_{2}\left(\frac{\left(\mathbb{E}_{W}[W]\right)^{2}+\mathbb{V}_{\mathbb{R}}[W]}{W^{2}}\right)} f(W) d W
\end{aligned}
$$

Onde $\theta_{m}$ é a aversão ao risco média efetiva. Na última integral, a fim de simplificar a notação, efetuamos um abuso de linguagem: $W$ representa a riqueza de um agente qualquer no instante inicial.

Tomando $W \sim \operatorname{Pareto}\left(\alpha, W_{\min }\right)$ (onde $\alpha>2$ é um parâmetro de "shape" da distribuição e se relaciona à distribuição de riqueza e $W_{\min }$ significa uma riqueza mínima) temos:

$f(W)=\frac{\alpha}{W_{\min }}\left(\frac{W_{\min }}{W}\right)^{\alpha+1}$, se $W \geq W_{\min }$ e $f(W)=0$, se $W<W_{\min }$

com

$$
\begin{gathered}
\mathbb{E}_{W}[W]=\frac{\alpha W_{\text {min }}}{\alpha-1} \\
\mathbb{V} \mathbb{R}_{W}[W]=\frac{\alpha W_{\text {min }}^{2}}{(\alpha-1)^{2}(\alpha-2)}
\end{gathered}
$$

Assim, teremos: 


$$
\begin{aligned}
& \frac{1}{\theta_{m}}=\int_{W_{\min }}^{+\infty} \frac{\left(c+\lambda_{1}\right)-\lambda_{1}\left(\frac{\mathbb{E}_{W}[W]}{W}\right)}{\left(b+\lambda_{2}\right)-\lambda_{2}\left(\frac{\left(\mathbb{E}_{W}[W]\right)^{2}+\mathbb{V A R}_{W}[W]}{W^{2}}\right)} f(W) d W \\
& =\int_{W_{\min }}^{+\infty} \frac{\left(c+\lambda_{1}\right)-\frac{\lambda_{1}}{W} \frac{\alpha W_{\min }}{\alpha-1}}{\left(b+\lambda_{2}\right)-\frac{\lambda_{2}}{W^{2}}\left(\frac{\alpha^{2} W_{\min }^{2}}{(\alpha-1)^{2}}+\frac{\alpha W_{\min }^{2}}{(\alpha-1)^{2}(\alpha-2)}\right)} \frac{\alpha}{W_{\min }}\left(\frac{W_{\min }}{W}\right)^{\alpha+1} d W \\
& =\alpha \int_{W_{\min }}^{+\infty} \frac{\left(c+\lambda_{1}\right)-\frac{\lambda_{1}}{W} \frac{\alpha W_{\min }}{\alpha-1}}{\left(b+\lambda_{2}\right)-\frac{\lambda_{2}}{W^{2}}\left(\frac{\alpha W_{\min }^{2}}{\alpha-2}\right)}\left(\frac{W_{\min }}{W}\right)^{\alpha+1} \frac{1}{W_{\min }} d W \\
& =\alpha \int_{W_{\min }}^{+\infty} \frac{\left(c+\lambda_{1}\right)-\frac{\lambda_{1} \alpha}{\alpha-1} \frac{W_{\min }}{W}}{\left(b+\lambda_{2}\right)-\frac{\lambda_{2} \alpha}{\alpha-2} \frac{W_{\min }^{2}}{W^{2}}}\left(\frac{W_{\min }}{W}\right)^{\alpha+1} \frac{1}{W_{\min }} d W
\end{aligned}
$$

Manipulando o último termo, obtemos:

$$
\begin{aligned}
\frac{1}{\theta_{m}} & =\alpha \int_{1}^{+\infty} \frac{\left(c+\lambda_{1}\right)-\frac{\lambda_{1} \alpha}{(\alpha-1) W}}{\left(b+\lambda_{2}\right)-\frac{\lambda_{2} \alpha}{(\alpha-2) W^{2}}} \frac{1}{W^{\alpha+1}} d W \\
& =\frac{\alpha(\alpha-2)}{\alpha-1} \int_{1}^{+\infty} \frac{(\alpha-1)\left(c+\lambda_{1}\right) W-\alpha \lambda_{1}}{\left((\alpha-2)\left(b+\lambda_{2}\right) W^{2}-\alpha \lambda_{2}\right) W^{\alpha}} d W
\end{aligned}
$$

Note que para a utilidade quadrática permaneça com sua primeira derivada positiva devemos limitar superiormente as riquezas $W$ tomando $W<\frac{c+\lambda_{1}}{b+\lambda_{2}}$, logo:

$$
\frac{1}{\theta_{m}}=\frac{\alpha(\alpha-2)}{\alpha-1} \int_{1}^{\frac{a+\lambda_{1}}{b+\lambda_{2}}} \frac{(\alpha-1)\left(c+\lambda_{1}\right) W-\alpha \lambda_{1}}{\left((\alpha-2)\left(b+\lambda_{2}\right) W^{2}-\alpha \lambda_{2}\right) W^{\alpha}} d W
$$

O índice de Gini (denotado por $G$ ) é um indicador bem conhecido em Economia para medir o nível de desigualdade (igualdade) de riqueza, ele varia entre 0 e 1 , onde $G=0$ significa igualdade total de distribuição de riqueza e $G=1$ significa completa desigualdade de distribuição de riqueza. Se a distribuição de riqueza segue a distribuição de Pareto: $W \sim \operatorname{Pareto}\left(\alpha, W_{\min }\right)$ (esta distribuição é usual para modelar distribuições de renda e riqueza), o parâmetro 
$\alpha$ pode ser relacionado ao índice de Gini, temos:

$$
G=\frac{1}{2 \alpha-1} \Leftrightarrow \alpha=\frac{1+G}{2 G}
$$

Note que $2<\alpha<+\infty \Leftrightarrow 0<G<1 / 3$, assim, o indicador está limitado a situações onde a distribuição de riqueza não é muito desigual, isto se deve a presença do termo de variância da distribuição de Pareto na integral que dá o inverso da aversão ao risco média efetiva.

Podemos relacionar explicitamente o índice de Gini e a aversão ao risco média, assim, sem fazer uso de aproximações temos (forma exata):

\section{Teorema: Fórmula da aversão ao risco média efetiva do mercado} Em nosso modelo, considerando as hipóteses feitas sobre o mercado, com a influência social representada pela função de utilidade generalizada quadrática e a estrutura de crenças heterogêneas representada por uma distribuição multivariada $\pi_{a}$, e ainda com a distribuição de riqueza dos agentes dada por uma Pareto de indice de Gini $G,(0<G<1 / 3)$ temos que a aversão ao risco média efetiva do mercado é:

$$
\frac{1}{\theta_{m}}=\frac{(1+G)(1-3 G)}{2 G(1-G)} \int_{1}^{\frac{c+\lambda_{1}}{b+\lambda_{2}}} \frac{(1-G)\left(c+\lambda_{1}\right) W-(1+G) \lambda_{1}}{\left((1-3 G)\left(b+\lambda_{2}\right) W^{2}-(1+G) \lambda_{2}\right) W^{\frac{1+G}{2 G}}} d W
$$

Esta integral pode ser calculada numericamente. Assim, tomando como valor base $b=1$, podemos observar de maneira gráfica a relação entre a aversão ao risco média efetiva do mercado $\theta_{m}$ em função do índice de Gini $G$ nas figuras 2 e 3 , para a relação exata em nosso modelo entre $\theta_{m}$ e $G$, para alguns valores de $\lambda_{1}, \lambda_{2}$ e $b$.

A partir da análise dos gráficos podemos relacionar $\theta_{m} \operatorname{com} \alpha$ e $G$.

Tomando os parâmetros constantes temos: aumento da desigualdade de riqueza $\Leftrightarrow G \searrow 1 \Leftrightarrow \alpha \nearrow+\infty \Rightarrow \theta_{m} \nearrow$, ou seja, um aumento da desigualdade de riqueza dos investidores (nossa população está restrita aos participantes do mercado) leva a uma diminuição da aversão ao risco média. 


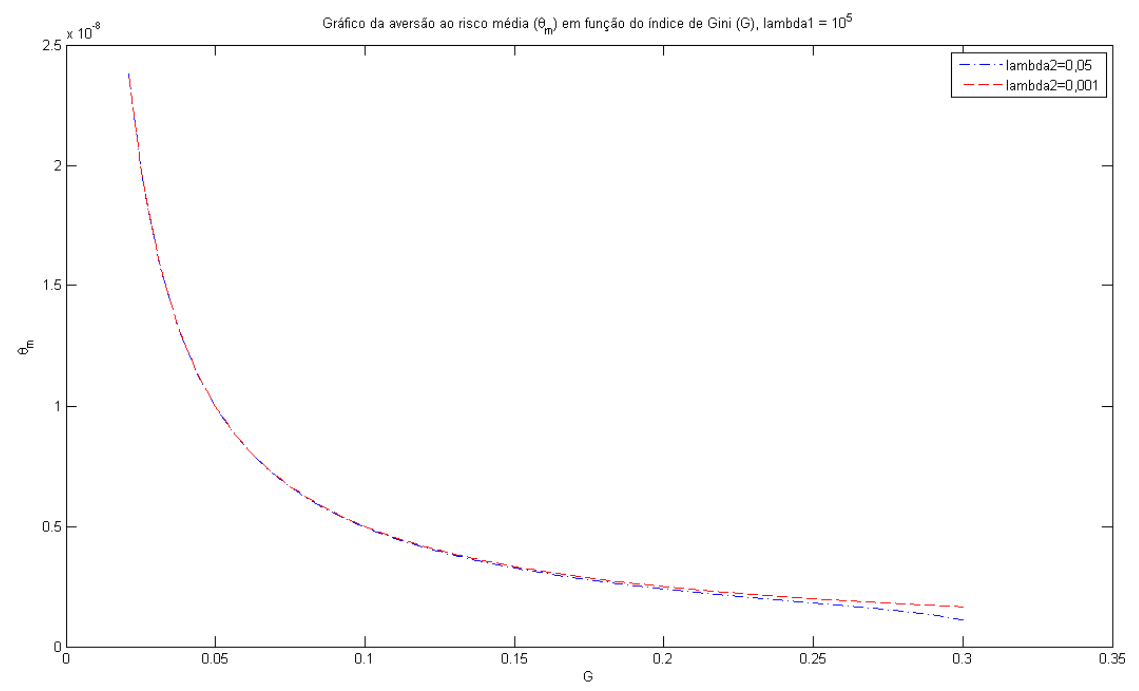

Figura 2: Aversão ao risco média efetiva $\left(\theta_{m}\right)$ versus índice de Gini $(\mathrm{G})$ $\left(\lambda_{1}=10^{5}\right)$ - Utilidade Quadrática

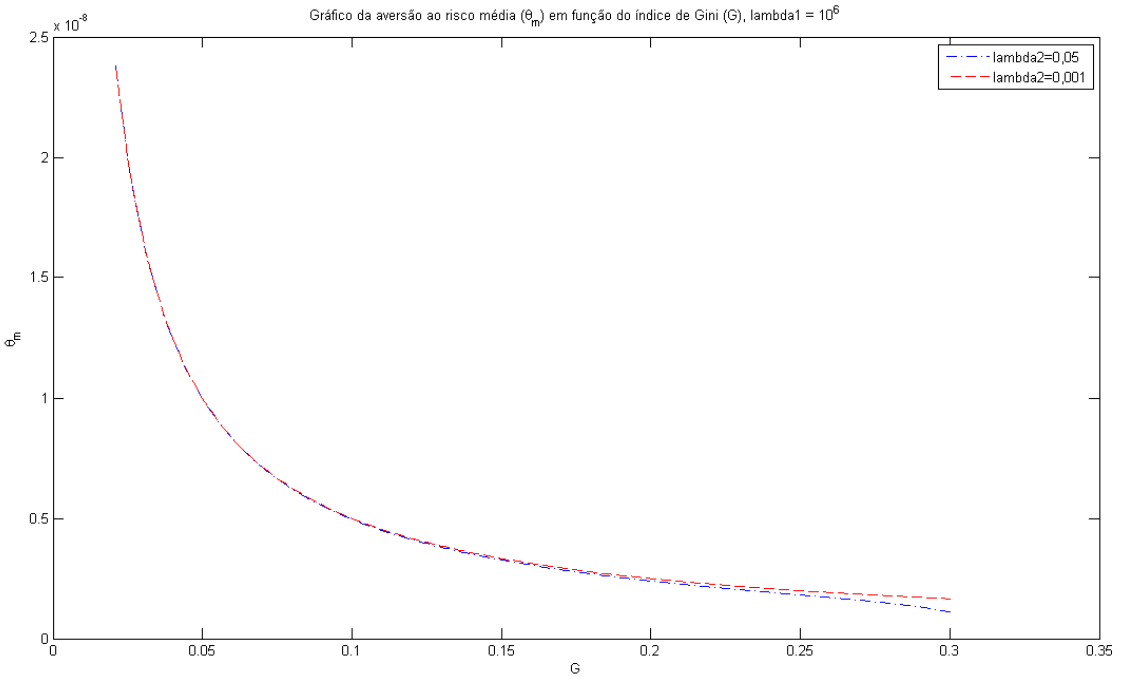

Figura 3: Aversão ao risco média efetiva $\left(\theta_{m}\right)$ versus índice de Gini $(\mathrm{G})$ $\left(\lambda_{1}=10^{6}\right)$ - Utilidade Quadrática 
Na equação que relaciona $r_{M}-r_{f} \operatorname{com} \theta_{M}$, temos que apenas $r_{M}$ e $\theta_{M}$ são endógenas, mais ainda, se $\theta_{M}$ diminui, $r_{M}-r_{f}$ aumenta o que só pode ocorrer se $r_{M}$ aumenta. Temos uma previsão de que um $\theta_{M}$ pequeno (menor que o tradicional) leva a um aumento do excesso de retorno do mercado.

Como sabemos, a volatilidade do mercado $\left(\sigma_{M}\right)$ é varia de modo inverso à aversão ao risco efetiva do mercado $\left(\theta_{M}\right)$, logo um aumento da desigualdade de riqueza dos investidores leva a um aumento da volatilidade do mercado (de forma mais acentuada do que a do modelo tradicional, onde esta relação inversa já existia, porém de modo independente da distribuição de riqueza).

No próximo capítulo vamos apresentar evidência empírica que corrobora os modelos e as conclusões apresentados neste e no capítulo anterior. 


\section{Evidências Empíricas}

Não é paradoxo dizer que nos nossos momentos de inspiração mais teórica podemos estar o mais próximo possível de nossas aplicações mais práticas.

A.N. Whitehead

\section{1 Índices}

Nossa previsão nos dois capítulos anteriores de que a volatilidade de um mercado financeiro aumenta com o aumento de sua concentração de riqueza. Aqui vamos mostrar algumas evidências empíricas que suportam esta conclusão.

Nosso modelo foi capaz de estabelecer uma ligação entre volatilidade e concentração de riqueza, porém existem outras variáveis que escapam de nossa modelagem (por exemplo, permanência dos investidores no mercado, mudanças de regulação, ...), de modo que a literatura aponta uma certa ambiguidade na relação entre estas duas variáveis (para uma discussão mais completa, veja [cetorelli] $[8])$.

Como é bem conhecido, a volatilidade do mercado $\left(\sigma_{M}\right)$ varia contrariamente a aversão ao risco média efetiva do mercado $\left(\theta_{m}\right)$. Assim, segundo nossa modelagem, um aumento da desigualdade de riqueza dos investidores leva a um aumento da volatilidade do mercado.

Apesar do índice de Gini ser altamente conveniente, por ser passível de ser obtido de modo analítico da distribuição de riqueza escolhida (Pareto), ele não é fácil de ser obtido empiricamente, a partir dos dados disponíveis. Assim, para verificar esta previsão, vamos utilizar o índice Herfindahl-Hirschman normalizado $\left(H H I^{*}\right)$ para a indústria de fundos mútuos como "proxy" para a desigualdade de riqueza dos investidores.

O índice Herfindahl-Hirschman $(H H I)$ é um índice tradicional de concentração de mercados (este índice é utilizado por exemplo, pela Securities and Exchange Commission (SEC), como um dos critérios para aceitar uma fusão ou aquisição de empresas no mercado americano) dado por: 


$$
H H I=\sum_{i=1}^{N} s_{i}^{2}
$$

onde $N$ é o número de companhias e $s_{i}$ é a medida da participação da companhia $i$ no mercado ("market share"). O índice $H H I$ varia de $1 / N$ até 1 , onde $1 / N$ significa uma distribuição uniforme de participação das companhias e 1 significa concentração total nas "mãos" de uma companhia.

O índice Herfindahl-Hirschman normalizado $\left(H H I^{*}\right)$ é dado por:

$$
H H I^{*}=\frac{H H I-1 / N}{1-1 / N}
$$

para $N>1$ e $H H I^{*}=1$, para $N=1$. Onde $N$ é o número de companhias no mercado e $H H I$ é o índice Herfindahl-Hirschman. O índice $H H I^{*}$ varia de 0 até 1 , sendo mais apropriado para medida da desigualdade de riqueza. Assim, como o índice de Gini, quanto mais próximo de 0, menos concentrado é o mercado e quanto mais próximo de 1 , maior é a concentração do mercado. Em [papadopoulos][20] nós temos uma discussão detalhada sobre a relação entre os índices de Gini, $H H I$ e $H H I^{*}$.

\subsection{Apresentação dos Dados e ANOVA}

Os dados sobre a volatilidade dos países são do artigo "Volatility and Stock Market Returns around the World" de S.X. Liang e K.C.J. Wei ([liang][17]) para 2005. O cálculo da volatilidade foi através da volatilidade do portfolio mercado que é o desvio padrão do retorno diário do portfolio mercado vezes $\sqrt{250 / 12}$ (os dados foram tomados em dólares americanos para efeito de padronização), mais detalhes podem ser vistos no artigo citado.

Os dados sobre concentração de riqueza são do artigo "Mutual Fund Industry Competition and Concentration: International Evidence" de M. Ferreira e S.B. Ramos([ferreira][11]). Dados sobre concentração de riqueza são muitos difíceis de adquirir, assim utilizamos uma "proxy" dada pela concentração de fundos. Uma vantagem de olhar para a concentração de fundos é que estes 
são muito mais estáveis (permanecem mais tempo como atuantes) como participantes do mercado do que investidores individuais. São utilizados dois tipos de dados: de (i) companhias administradoras (que supervisionam o desempenho de outras empresas que prestam serviços às operações dos fundos) e de (ii) companhias gestoras de carteiras ("portfolios"). Mais detalhes podem ser vistos no artigo citado.

A seguir, os dados utilizados de vários países.

Tabela 1: Companhias gestoras de carteiras ("portfolios") por países

\begin{tabular}{|c|c|c|c|c|}
\hline Países & $N_{m}(1)$ & $H H I m(1)$ & $H_{\text {HIm }}^{*}$ & Volatilidade $(\sigma)(2)$ \\
\hline Austrália & 190 & 0.066 & 0.061058 & 0.0485 \\
Áustria & 174 & 0.087 & 0.081723 & 0.0429 \\
Canadá & 155 & 0.051 & 0.044838 & 0.0354 \\
Dinamarca & 40 & 0.090 & 0.066667 & 0.0504 \\
Finlândia & 62 & 0.135 & 0.120820 & 0.0814 \\
França & 297 & 0.047 & 0.043780 & 0.0578 \\
Alemanha & 192 & 0.070 & 0.065131 & 0.0510 \\
Itália & 78 & 0.067 & 0.054883 & 0.0624 \\
Japão & 68 & 0.085 & 0.071343 & 0.0495 \\
Holanda & 51 & 0.109 & 0.091180 & 0.0549 \\
Noruega & 28 & 0.133 & 0.100889 & 0.0637 \\
Portugal & 18 & 0.141 & 0.090471 & 0.0471 \\
Singapura & 48 & 0.066 & 0.046128 & 0.0523 \\
Espanha & 115 & 0.103 & 0.095132 & 0.0583 \\
Suécia & 48 & 0.120 & 0.101277 & 0.0627 \\
Suiça & 136 & 0.091 & 0.084267 & 0.0508 \\
Reino Unido & 429 & 0.023 & 0.020717 & 0.0496 \\
\hline
\end{tabular}

(1) dados do artigo [ferreira][11]

(2) dados do artigo [liang][17]

Tabela 2: Análise de Regressão

A equação de regressão é $\sigma=0.0383+0.215$ H H Im $^{*}$

\begin{tabular}{|c|c|c|c|c|}
\hline Preditor & Coef & SE Coef & T & P \\
\hline Constante & 0.038331 & 0.006463 & 5.93 & 0.000 \\
H H Im $^{*}$ & 0.21533 & 0.08370 & 2.57 & 0.021 \\
\hline $\mathrm{S}=0.00872736$ & $\mathrm{R}-\mathrm{Sq}$ & 0.306 & $\mathrm{R}-\mathrm{Sq}(\mathrm{adj})$ & 0.26 \\
\hline
\end{tabular}


Tabela 3: Análise de Variância

\begin{tabular}{|c|c|c|c|c|c|}
\hline Source & DF & SS & MS & F & P \\
\hline Regressão & 1 & 0.00050404 & 0.00050404 & 6.62 & 0.021 \\
Erro Residual & 15 & 0.00114250 & 0.00007617 & & \\
Total & 16 & 0.00164654 & & & \\
\hline
\end{tabular}

Tabela 4: Companhias Administradoras por Países

\begin{tabular}{|c|c|c|c|c|}
\hline Países & $N_{a}(1)$ & $H H I a(1)$ & $H_{H I a}$ & Volatilidade $(\sigma)(2)$ \\
\hline Austrália & 121 & 0.043 & 0.035025 & 0.0485 \\
Áustria & 55 & 0.111 & 0.094537 & 0.0429 \\
Canadá & 96 & 0.063 & 0.053137 & 0.0354 \\
Dinamarca & 23 & 0.170 & 0.132273 & 0.0504 \\
Finlândia & 24 & 0.191 & 0.155826 & 0.0814 \\
França & 268 & 0.066 & 0.062502 & 0.0578 \\
Alemanha & 137 & 0.071 & 0.064169 & 0.0510 \\
Itália & 56 & 0.069 & 0.052073 & 0.0624 \\
Japão & 68 & 0.085 & 0.071343 & 0.0495 \\
Holanda & 37 & 0.128 & 0.103778 & 0.0549 \\
Noruega & 20 & 0.141 & 0.095789 & 0.0637 \\
Portugal & 18 & 0.141 & 0.090471 & 0.0471 \\
Singapura & 41 & 0.062 & 0.038550 & 0.0523 \\
Espanha & 108 & 0.103 & 0.094617 & 0.0583 \\
Suécia & 46 & 0.121 & 0.101467 & 0.0627 \\
Suiça & 52 & 0.142 & 0.125176 & 0.0508 \\
Reino Unido & 196 & 0.040 & 0.035077 & 0.0496 \\
\hline
\end{tabular}

(1) dados do artigo [ferreira][11]

(2) dados do artigo [liang][17]

Tabela 5: Análise de Regressão

A equação de regressão é $\sigma=0.0428+0.136 H H I a^{*}$

\begin{tabular}{|c|c|c|c|c|}
\hline Preditor & Coef & SE Coef & $\mathrm{T}$ & $\mathrm{P}$ \\
\hline Constante & 0.042794 & 0.005823 & 7.35 & 0.000 \\
H HIa $^{*}$ & 0.13601 & 0.06502 & 2.09 & 0.054 \\
\hline $\mathrm{S}=0.00921838$ & $\mathrm{R}-\mathrm{Sq}$ & 0.226 & $\mathrm{R}-\mathrm{Sq}(\mathrm{adj})$ & 0.174 \\
\hline
\end{tabular}

Tabela 6: Análise de Variância

\begin{tabular}{|c|c|c|c|c|c|}
\hline Source & DF & SS & MS & F & P \\
\hline Regressão & 1 & 0.00037186 & 0.00037186 & 4.38 & 0.054 \\
Erro Residual & 15 & 0.00127468 & 0.00008498 & & \\
Total & 16 & 0.00164654 & & & \\
\hline
\end{tabular}

A seguir as figuras com dados das tabelas que apresentam a relação entre a volatilidade $(\sigma)$ e o índice Herfindahl-Hirschman normalizado $\left(H H I a^{*}\right)$ : 


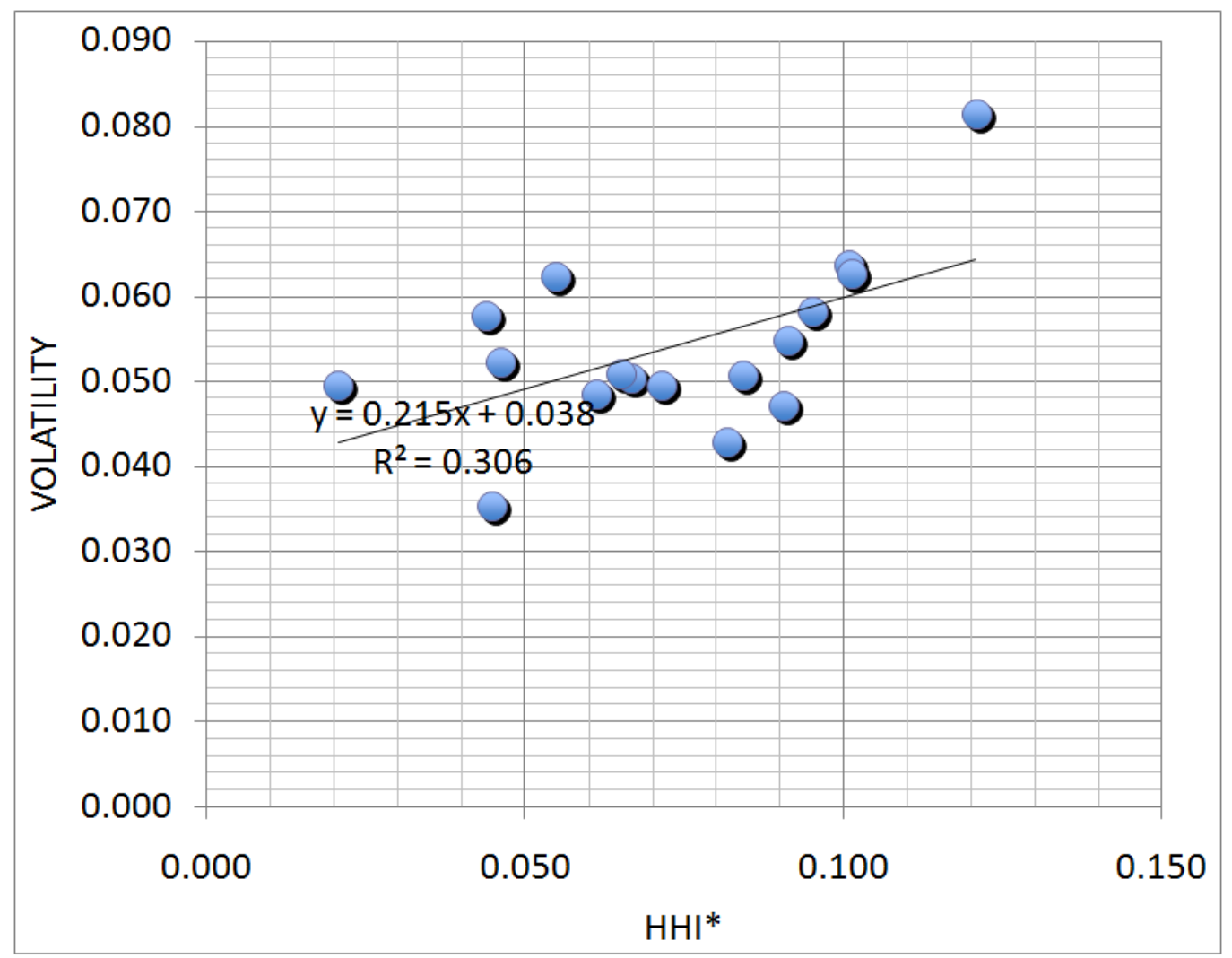

Figura 4: Volatilidade $(\sigma)$ versus índice Herfindahl-Hirschman normalizado $\left(\right.$ HHIm $\left.^{*}\right)$

A partir da teoria desenvolvida, nossa previsão é que a medida que o índice Herfindahl-Hirschman normalizado $\left(H H I^{*}\right)$ aumenta (maior concentração do mercado) maior será a volatilidade observada, ou seja, o coeficiente de inclinação da regressão deve ser positivo em ambos os casos.

De fato, isto foi o observado, no primeiro caso a regressão obtida foi $\sigma=$ $0.0383+0.215$ H H Im $^{*}$, sendo o coeficiente linear igual a 0.215 (com estatística $T=2.57$ e $p-$ valor $=0.021)$ e $R^{2}=0.306$.

No segundo caso a regressão obtida foi $\sigma=0.0428+0.136 H H I a^{*}$, sendo o coeficiente linear igual a $0.136($ com estatística $T=2.09$ e $p-$ valor $=0.054)$ e $R^{2}=0.226$.

A partir dos resultados da análise da regressão realizada (ANOVA) nós temos evidência empírica que suporta nossa abordagem. 


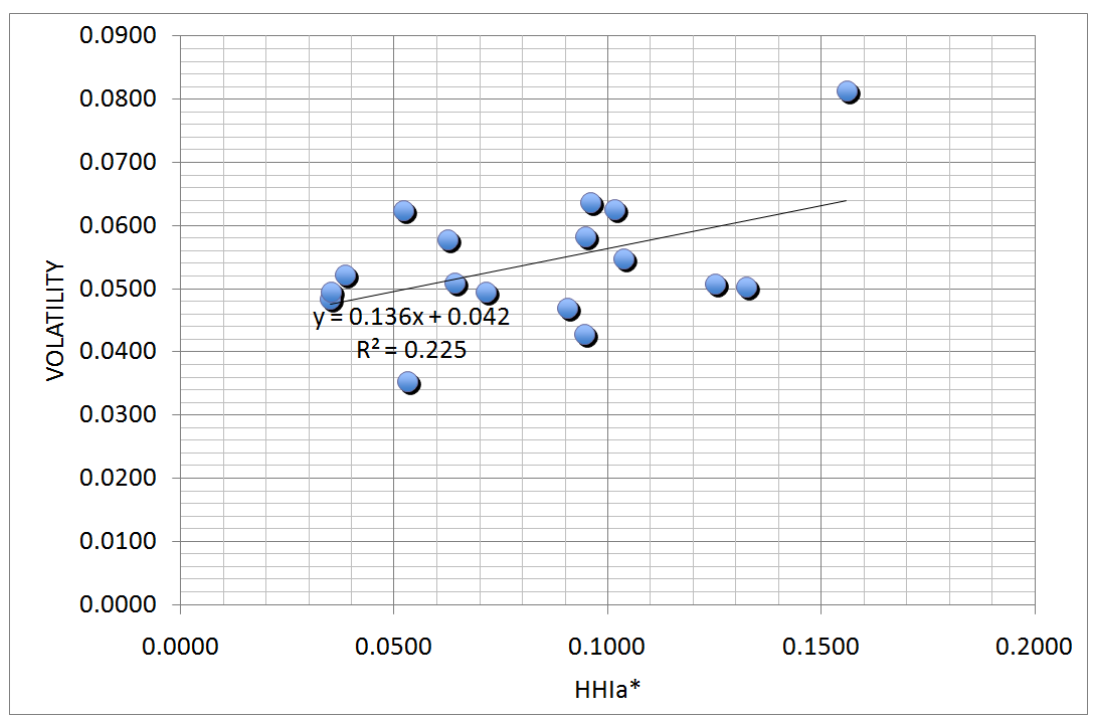

Figura 5: Volatilidade $(\sigma)$ versus índice Herfindahl-Hirschman normalizado (HHIa*) 


\section{Considerações sobre "Puzzles" Econômicos}

We make models to abstract reality. But there is a meta-model beyond the model that assures us that the model will eventually fail. Models fail because they fail to incorporate the interrelationships that exist in the real world.

Myron Scholes

Speech at NYU/IXIS conference on hedge funds, 2005

Na citação que abre este capítulo, Scholes comenta os problemas que eventualmente surgem com modelos, por deixarem de incorporar aspectos do mundo real. Como relatado no capítulo 2, algumas destas falhas surgiram nos anos 80 para as Finanças tradicionais, agora, vamos mostrar como o "framework" desenvolvido aqui pode ajudar a lidar com os "puzzles" observados.

\subsection{Os Puzzles}

No capítulo 3 apresentamos o conceito de função utilidade generalizada para a função de utilidade potência. De posse desta função somos levados a considerar a presença da influência social em alguns puzzles econômicos bem conhecidos, o "Equity Premium Puzzle" (EPP) e o "Riskfree Rate Puzzle"(RRP).

Como dito no capítulo 2, Prescott e Mehra publicaram um artigo em 1985, onde desenvolveram um modelo de equilíbrio geral, baseado em agente representativo com utilidade de consumo aditiva e separável no tempo e com aversão ao risco relativa constante, onde obtiveram a seguinte equação:

$$
\frac{r_{M}-r_{f}}{\sigma_{M}}=\gamma \sqrt{\mathbb{V} \mathbb{A} \mathbb{R}_{t}\left[\Delta \ln \left(c^{t+1}\right)\right]}
$$

onde $\gamma$ é igual ao coeficiente de aversão ao risco relativo e também é igual, no modelo, a elasticidade de substituição intertemporal. Os outros parâmetros são: $r_{M}$ o retorno esperado do mercado (conforme definido nos capítulos sobre o CAPM), $r_{f}$ a taxa de juros livre de risco, $\sigma_{M}$ o desvio padrão da volatilidade do mercado e $\mathbb{V} \mathbb{R}_{t}\left[\Delta \ln \left(c^{t+1}\right)\right]$ a variância no tempo da variação do 
log-consumo (este termo será definido de maneira mais precisa nas próximas seções).

Comparando com dados do mercado acionário, Prescott e Mehra, encontraram que $\gamma$ deveria ser algo entre 30 e 40, o que foi considerado extremamente elevado (era esperado algo entre 1 e 2, com no máximo 10). Este problema foi denominado "Equity Premium Puzzle" (EPP).

Um exemplo apresentado por [thaler][30] e [xu][31] para entender o significado deste $\gamma$ elevado é o seguinte: suponha um jogo, onde o jogador tem probabilidade $1 / 2$ de ganhar e dobrar sua riqueza e probabilidade $1 / 2$ de perder e ter sua riqueza reduzida pela metade, quanto o jogador estaria disposto a pagar para evitar tal aposta, se tiver um coeficiente de aversão ao risco igual a 30 ? $49 \%$ da riqueza dele, o que soa como absurdo, pois em caso de perda, ele perde metade de sua riqueza.

Muitas foram as tentativas desde então de explicar esta discrepância, bem como verificar sua presença em outros períodos e outros países. Discussões a respeito da busca de soluções podem ser encontradas em [thaler][30], [xu][31] e [bonomo][5] (em particular, [xu][31] faz uma análise de propostas baseadas em Finanças Comportamentais, porém com abordagens diferentes da apresentada nesta tese). Verificações empíricas em diversos mercados, em particular no Brasil, podem ser encontradas em [guimaraes][12], [samanez][23] e $[\mathrm{xu}][31]$.

No decorrer das investigações, foi encontrado por Weil em 1989, utilizando a mesma modelo de Prescott e Mehra, outro puzzle: "Riskfree Rate Puzzle"(RRP). Weil obteve a seguinte equação:

$$
r^{f}=-\ln \beta_{a}+\gamma \mathbb{E}_{t}\left[\Delta \ln \left(c^{t+1}\right)\right]-\frac{\gamma^{2}}{2} \mathbb{V} \mathbb{R} \mathbb{R}_{t}\left[\Delta \ln \left(c^{t+1}\right)\right]
$$

onde $\beta_{a}$ é o fator subjetivo de desconto e $\mathbb{E}_{t}\left[\Delta \ln \left(c^{t+1}\right)\right]$ é o valor esperado no tempo da variação do log-consumo e as demais variáveis são como definidas anteriormente.

Novamente, quando utilizados dados do mercado americano, foram obtidos valores incongruentes para $\beta_{a}$. Desde então, este novo "puzzle"também foi motivo de investigações teóricas e empíricas (veja [bonomo][5], [thaler][30] e [guimaraes][12]). 
Neste capítulo vamos repetir a modelagem que levou aos "puzzles", porém fazendo uso da utilidade generalizada que incorpora a presença de influência social e mostrar que esta pode ter impacto na solução e compreensão dos "puzzles" (ainda que parcial).

\subsection{Função de Utilidade do Consumo}

Vamos supor que a utilidade do consumo de um agente representativo $a$ segue uma utilidade do tipo potência generalizada. Para o consumo em $t, c_{a}^{t}$, temos:

$$
\begin{aligned}
U\left(c_{a}^{t}, c_{-a}^{t}\right) & =\frac{\left(c_{a}^{t}\right)^{1-\gamma}}{1-(\gamma+\lambda)}\left(\prod_{j=1 j \neq a}^{m}\left(\frac{c_{a}^{t}}{c_{j}^{t}}\right)^{\frac{1}{m-1}}\right)^{-\lambda} \\
& =\frac{\left(c_{a}^{t}\right)^{1-\gamma}}{1-(\gamma+\lambda)}\left(c_{a}^{t}\right)^{-\lambda}\left(\bar{c}_{-a}^{t}\right)^{\lambda} \\
& =\frac{\left(c_{a}^{t}\right)^{1-(\gamma+\lambda)}}{1-(\gamma+\lambda)}\left(\bar{c}_{-a}^{t}\right)^{\lambda}
\end{aligned}
$$

Onde

$$
\bar{c}_{-a}^{t}=\prod_{j=1 j \neq a}^{m}\left(c_{j}^{t}\right)^{\frac{1}{m-1}}
$$

ou seja, $\bar{c}_{-a}^{t}$ é a média geométrica dos consumos $c_{j}^{t}$ dos demais agentes, diferentes de $a$. Os termos $\gamma$ e $\lambda$ são positivos e tais que $\gamma>>\lambda$ e $\gamma+\lambda<1$.

Neste caso estamos supondo que todas os valores $c_{j}, 1 \leq j \leq m$ são positivos.

Uma hipótese bastante importante de nosso modelo, é a crença heterogênea dos agentes em relação ao consumo dos demais, isso é representado da seguinte forma: o investidor $a$ acredita numa certa distribuição de probabilidades para o consumo dos demais agentes para cada ativo $i$. 
A estrutura probabilística das crenças de $a$ quanto ao consumo do ativo $i$ pelos demais investidores é uma distribuição $\left(\pi_{a}\right)$ formada pelo produto direto de duas distribuições, uma que representa as crenças de $a$ quanto ao consumo dos demais no instante $t\left(\pi_{a, t}\right)$ e outra que representa as crenças de $a$ quanto ao consumo dos demais no instante $t+1\left(\pi_{a, t+1}\right)$. Assim, temos:

$$
\pi_{a}=\pi_{a, t} \bigotimes \pi_{a, t+1}
$$

A função de utilidade potência efetiva $\left(U_{e}\left(c_{a}^{t}\right)\right)$ é dada por:

$$
\begin{aligned}
U_{e}\left(c_{a}^{t}\right) & =\mathbb{E}_{\pi_{a}}\left[U\left(c_{a}^{t}, c_{-a}^{t}\right)\right] \\
& =\frac{\left(c_{a}^{t}\right)^{1-(\gamma+\lambda)}}{1-(\gamma+\lambda)} \mathbb{E}_{\pi_{a}}\left[\left(\bar{c}_{-a}^{t}\right)^{\lambda}\right]
\end{aligned}
$$

Para uso nas próximas seções, vamos calcular a primeira derivada da utilidade efetiva:

$$
\begin{aligned}
\frac{d U_{e}\left(c_{a}^{t}\right)}{d c_{a}^{t}} & =\frac{(1-(\gamma+\lambda))\left(c_{a}^{t}\right)^{1-(\gamma+\lambda)-1}}{1-(\gamma+\lambda)} \mathbb{E}_{\pi_{a}}\left[\left(\bar{c}_{-a}^{t}\right)^{\lambda}\right] \\
& =\left(c_{a}^{t}\right)^{-(\gamma+\lambda)} \mathbb{E}_{\pi_{a}}\left[\left(\bar{c}_{-a}^{t}\right)^{\lambda}\right]
\end{aligned}
$$

Analogamente, para o consumo em $t+1, c_{a}^{(t+1)}$, temos:

$$
U\left(c_{a}^{t+1}, c_{-a}^{t+1}\right)=\frac{\left(c_{a}^{t+1}\right)^{1-(\gamma+\lambda)}}{1-(\gamma+\lambda)}\left(\bar{c}_{-a}^{t+1}\right)^{\lambda}
$$

Onde, $\bar{c}_{-a}^{t+1}$ é a média geométrica dos consumos $c_{j}^{t+1}$ dos demais agentes, diferentes de $a$.

A função de utilidade potência efetiva $\left(U_{e}\left(c_{a}^{t+1}\right)\right)$ é dada por: 


$$
U_{e}\left(c_{a}^{t+1}\right)=\frac{\left(c_{a}^{t+1}\right)^{1-(\gamma+\lambda)}}{1-(\gamma+\lambda)} \mathbb{E}_{\pi_{a}}\left[\left(\bar{c}_{-a}^{t+1}\right)^{\lambda}\right]
$$

Vamos calcular a primeira derivada da utilidade efetiva:

$$
\frac{d U_{e}\left(c_{a}^{t+1}\right)}{d c_{a}^{t+1}}=\left(c_{a}^{t+1}\right)^{-(\gamma+\lambda)} \mathbb{E}_{\pi_{a}}\left[\left(\bar{c}_{-a}^{t+1}\right)^{\lambda}\right]
$$

\subsection{Modelando o Fator de Desconto Estocástico}

Vamos partir inicialmente da seguinte questão: qual o valor em $t$ de um ativo com valor $S^{t+1}$ em $t+1$ ?

Seguindo o tratamento tradicional, suponha que a utilidade efetiva em termos do consumo em $t$ e $t+1$ de um investidor típico $a$ seja dada por uma função de utilidade aditiva separável no tempo da forma:

$$
U_{e}\left(c_{a}^{t}, c_{a}^{t+1}\right)=U_{e}\left(c_{a}^{t}\right)+\beta_{a} \mathbb{E}_{t}\left[U_{e}\left(c_{a}^{t+1}\right)\right]
$$

onde $\beta_{a}<1$ é o fator subjetivo de desconto (representa a impaciência de consumir).

Aqui devemos observar que a influência social já está incorporada nos termos $U_{e}\left(c_{a}^{t}\right)$ e $\mathbb{E}_{t}\left[U_{e}\left(c_{a}^{t+1}\right)\right]$, de modo que não há necessidade de criar outro tipo de função de utilidade generalizada, esta é obtida como uma combinação linear das outras. Outro ponto importante é que estamos considerando apenas o caso onde a variação de consumo é de apenas um período.

Considere a seguinte situação: o investidor tem dotação orçamentária ("endowment") $e_{a}^{t}$ e $e_{a}^{t+1}$ respectivamente em $t$ e $t+1$, e pode comprar ou vender livremente o ativo $S^{t+1}$ pelo valor $S^{t}$. Pergunta: quanto comprar ou vender? Podemos descrever este problema do seguinte modo: 


$$
\begin{aligned}
\max _{x_{a}} & U_{e}\left(c_{a}^{t}, c_{a}^{t+1}\right)=\max _{x_{a}} U_{e}\left(c_{a}^{t}\right)+\beta_{a} \mathbb{E}_{t}\left[U_{e}\left(c_{a}^{t+1}\right)\right] \\
c_{a}^{t} & =e_{a}^{t}-S^{t} x_{a} \\
c_{a}^{t+1} & =e_{a}^{t+1}+S^{t+1} x_{a}
\end{aligned}
$$

Temos

$$
\begin{aligned}
\frac{d U_{e}\left(c_{a}^{t}\right)}{d x_{a}} & =\frac{d\left(U_{e}\left(c_{a}^{t}\right)+\beta_{a} \mathbb{E}_{t}\left[U_{e}\left(c_{a}^{t+1}\right)\right]\right)}{d x_{a}} \\
& =-S^{t} \frac{d U_{e}\left(c_{a}^{t}\right)}{d c_{a}^{t}}+\beta_{a} \mathbb{E}_{t}\left[S^{t+1} \frac{d\left(U_{e}\left(c_{a}^{t+1}\right)\right)}{d c_{a}^{t+1}}\right]
\end{aligned}
$$

No equilíbrio podemos impor as condições de primeira ordem (primeira derivada nula) temos:

$$
\begin{aligned}
S^{t} \frac{U_{e}\left(c_{a}^{t}\right)}{d c_{a}^{t}} & =\beta_{a} \mathbb{E}_{t}\left[S^{t+1} \frac{d\left(U_{e}\left(c_{a}^{t+1}\right)\right)}{d c_{a}^{t+1}}\right] \Longleftrightarrow \\
S^{t} & =\mathbb{E}_{t}\left[\beta_{a} \frac{\frac{d\left(U_{e}\left(c_{a}^{t+1}\right)\right)}{d c_{a}^{t+1}}}{\frac{d U_{e}\left(c_{a}^{t}\right)}{d c_{a}^{t}}} S^{t+1}\right]
\end{aligned}
$$

Esta equação pode ser interpretada como o preço do ativo que paga $S^{t+1}$ para dado consumo em $c_{a}^{t}$ e $c_{a}^{t+1}$. Podemos definir o fator de desconto estocástico (também chamado de taxa marginal de substituição) por:

$$
M^{t+1}=\beta_{a} \frac{\frac{d U_{e}\left(c_{a}^{t+1}\right)}{d c_{a}^{t+1}}}{\frac{d U_{e}\left(c_{a}^{t}\right)}{d c_{a}^{t}}}
$$

O que permite reescrever a equação de apreçamento como: 


$$
S^{t}=\mathbb{E}_{t}\left[M^{t+1} S^{t+1}\right]
$$

Da equação acima, podemos dizer que o fator de desconto estocástico generaliza o fator de desconto da Matemática Financeira elementar.

Neste capítulo vamos trabalhar com o conceito de retorno bruto, dado por:

$$
R^{i}=\frac{S^{t+1}}{S^{t}}
$$

onde $S^{t}$ é o valor do ativo $i$ no instante inicial e $S^{t+1}$ é o valor do ativo $i$ no instante seguinte, considerados.

Agora, considere um ativo livre de risco com valor $S^{t}$ em $t$ e com retorno bruto $R^{f}$, pela equação de apreçamento, temos:

$$
\begin{aligned}
S^{t} & =\mathbb{E}_{t}\left[M^{t+1} S^{t+1}\right] \Longleftrightarrow \\
\frac{S^{t}}{S^{t}} & =\mathbb{E}_{t}\left[M^{t+1} \frac{S^{t+1}}{S^{t}}\right] \Longleftrightarrow \\
1 & =\mathbb{E}_{t}\left[M^{t+1} R^{f}\right] \Longleftrightarrow \\
R^{f} \mathbb{E}_{t}\left[M^{t+1}\right] & =1 \Longleftrightarrow \\
R^{f} & =\frac{1}{\mathbb{E}_{t}\left[M^{t+1}\right]}
\end{aligned}
$$

\subsection{Aplicando a Utilidade Generalizada no Fator de Desconto Estocástico}

Considerando a função de utilidade do tipo potência generalizada, temos que o fator de desconto estocástico $\left(M^{t+1}\right)$ é dado por: 


$$
\begin{aligned}
M^{t+1} & =\beta_{a} \frac{\frac{d U_{e}\left(c_{a}^{t+1}\right)}{d c_{a}^{t+1}}}{\frac{d U_{e}\left(c_{a}^{t}\right)}{d c_{a}^{t}}} \\
& =\beta_{a} \frac{\left(c_{a}^{t+1}\right)^{-(\gamma+\lambda)} \mathbb{E}_{\pi_{a}}\left[\left(\bar{c}_{-a}^{t+1}\right)^{\lambda}\right]}{\left(c_{a}^{t}\right)^{-(\gamma+\lambda)} \mathbb{E}_{\pi_{a}}\left[\left(\bar{c}_{-a}^{t}\right)^{\lambda}\right]} \\
& =\beta_{a}\left(\frac{c_{a}^{t+1}}{c_{a}^{t}}\right)^{-(\gamma+\lambda)} \frac{\mathbb{E}_{\pi_{a}}\left[\left(\bar{c}_{-a}^{t+1}\right)^{\lambda}\right]}{\mathbb{E}_{\pi_{a}}\left[\left(\bar{c}_{-a}^{t}\right)^{\lambda}\right]}
\end{aligned}
$$

\section{Temos}

$$
\begin{aligned}
& M^{t+1}=\exp \left[\ln \left(\beta_{a}\left(\frac{c_{a}^{t+1}}{c_{a}^{t}}\right)^{-(\gamma+\lambda)} \frac{\mathbb{E}_{\pi_{a}}\left[\left(\bar{c}_{-a}^{t+1}\right)^{\lambda}\right]}{\mathbb{E}_{\pi_{a}}\left[\left(\bar{c}_{-a}^{t}\right)^{\lambda}\right]}\right)\right] \\
& =\exp -\left(\delta+(\gamma+\lambda) \ln \left(\frac{c_{a}^{t+1}}{c_{a}^{t}}\right)-\ln \left(\frac{\mathbb{E}_{\pi_{a}}\left[\left(\bar{c}_{-a}^{t+1}\right)^{\lambda}\right]}{\mathbb{E}_{\pi_{a}}\left[\left(\bar{c}_{-a}^{t}\right)^{\lambda}\right]}\right)\right) \\
& =\exp -\left(\delta+(\gamma+\lambda) \ln \left(\frac{c_{a}^{t+1}}{c_{a}^{t}}\right)\right) \\
& \times \exp -\left(-\ln \left(\mathbb{E}_{\pi_{a}}\left[\left(\bar{c}_{-a}^{t+1}\right)^{\lambda}\right]\right)+\ln \left(\mathbb{E}_{\pi_{a}}\left[\left(\bar{c}_{-a}^{t}\right)^{\lambda}\right]\right)\right)
\end{aligned}
$$

Onde temos $\beta=\exp (-\delta)$, o termo $\delta$, representa a impaciência para consumir.

Queremos obter um termo limitante inferior que é mais fácil de manipular, para isso vamos fazer uso da desigualdade de Jensen: 
Desigualdade de Jensen: Seja $\phi$ uma função estritamente convexa (respectivamente, estritamente côncava) de uma variável real. Se a variável aleatória $X$ é integrável, então temos $\mathbb{E}[\phi(X)]>\phi(\mathbb{E}[X])$ (respectivamente, $\mathbb{E}[\phi(X)]<\phi(\mathbb{E}[X]))$.

Note que as funções $\ln x$ e $x^{\lambda}$ (para $0<\lambda<1$ ) são estritamente côncavas.

Assim temos, aplicando a desigualdade de Jensen sucessivamente, para o instante $t+1$ :

$$
\begin{aligned}
& \mathbb{E}_{\pi_{a}}\left[\left(\bar{c}_{-a}^{t+1}\right)^{\lambda}\right]<\left(\mathbb{E}_{\pi_{a}}\left[\left(\bar{c}_{-a}^{t+1}\right)\right]\right)^{\lambda} \Rightarrow \\
& \ln \left(\mathbb{E}_{\pi_{a}}\left[\left(\bar{c}_{-a}^{t+1}\right)^{\lambda}\right]\right)<\ln \left(\left(\mathbb{E}_{\pi_{a}}\left[\left(\bar{c}_{-a}^{t+1}\right)\right]\right)^{\lambda}\right) \Rightarrow \\
&-\ln \left(\mathbb{E}_{\pi_{a}}\left[\left(\bar{c}_{-a}^{t+1}\right)^{\lambda}\right]\right)>-\lambda \ln \left(\left(\mathbb{E}_{\pi_{a}}\left[\left(\bar{c}_{-a}^{t+1}\right)\right]\right)\right)>-\lambda \mathbb{E}_{\pi_{a}}\left[\ln \left(\bar{c}_{-a}^{t+1}\right)\right]
\end{aligned}
$$

Para o instante $t$ temos:

$$
\begin{aligned}
& \ln \left(\mathbb{E}_{\pi_{a}}\left[\left(\bar{c}_{-a}^{t}\right)^{\lambda}\right]\right)>\mathbb{E}_{\pi_{a}}\left[\ln \left(\left(\bar{c}_{-a}^{t}\right)^{\lambda}\right)\right]=\lambda \mathbb{E}_{\pi_{a}}\left[\ln \left(\bar{c}_{-a}^{t}\right)\right] \Rightarrow \\
& \ln \left(\mathbb{E}_{\pi_{a}}\left[\left(\bar{c}_{-a}^{t}\right)^{\lambda}\right]\right)>\lambda \mathbb{E}_{\pi_{a}}\left[\ln \left(\bar{c}_{-a}^{t}\right)\right]
\end{aligned}
$$

Juntando as duas últimas desigualdades obtemos:

$$
\begin{aligned}
\mu^{t+1} & =(\gamma+\lambda) \ln \left(\frac{c_{a}^{t+1}}{c_{a}^{t}}\right)-\ln \left(\mathbb{E}_{\pi_{a}}\left[\left(\bar{c}_{-a}^{t+1}\right)^{\lambda}\right]\right)+\ln \left(\mathbb{E}_{\pi_{a}}\left[\left(\bar{c}_{-a}^{t}\right)^{\lambda}\right]\right) \\
& >(\gamma+\lambda) \ln \left(\frac{c_{a}^{t+1}}{c_{a}^{t}}\right)-\lambda \mathbb{E}_{\pi_{a}}\left[\ln \left(\bar{c}_{-a}^{t+1}\right)\right]+\lambda \mathbb{E}_{\pi_{a}}\left[\ln \left(\bar{c}_{-a}^{t}\right)\right] \\
& =(\gamma+\lambda) \ln \left(\frac{c_{a}^{t+1}}{c_{a}^{t}}\right)-\lambda \mathbb{E}_{\pi_{a}}\left[\ln \left(\bar{c}_{-a}^{t+1}\right)-\ln \left(\bar{c}_{-a}^{t}\right)\right] \\
& =(\gamma+\lambda) \ln \left(\frac{c_{a}^{t+1}}{c_{a}^{t}}\right)-\lambda \mathbb{E}_{\pi_{a}}\left[\ln \left(\frac{\bar{c}_{-a}^{t+1}}{\bar{c}_{-a}^{t}}\right)\right]
\end{aligned}
$$


No tratamento tradicional dos "puzzles" econômicos, o termo correspondente a $\mu^{t+1}$ é suposto se comportar como uma variável aleatória normal (o consumo cresce como uma log-normal). Aqui aplicamos a desigualdade de Jensen para obter um termo limitante inferior que é mais fácil de manipular e mais razoável de se fazer hipóteses, então vamos colocar tal hipótese em nosso limitante.

Sejam $\Delta \ln \left(c^{t+1}\right)=\ln \left(\frac{c_{a}^{t+1}}{c_{a}^{t}}\right)$ e $\Delta \ln \left(\bar{c}^{t+1}\right)=\mathbb{E}_{\pi_{a}}\left[\ln \left(\frac{\bar{c}_{-a}^{t+1}}{\bar{c}_{-a}^{t}}\right)\right]$, agora, por hipótese temos que $\Delta \ln \left(c^{t+1}\right)$ e $\Delta \ln \left(\bar{c}^{t+1}\right)$ seguem uma distribuição normal.

Assim, a variável $Y$ :

$$
Y=\exp \left(-(\gamma+\lambda) \Delta \ln \left(c^{t+1}\right)+\lambda \Delta \ln \left(\bar{c}^{t+1}\right)\right)
$$

segue uma distribuição log-normal com

$$
\begin{aligned}
& \mathbb{E}_{t}[Y]=-(\gamma+\lambda) \mathbb{E}_{t}\left[\Delta \ln \left(c^{t+1}\right)\right]+\lambda \mathbb{E}_{t}\left[\Delta \ln \left(\bar{c}^{t+1}\right)\right]+\frac{(\gamma+\lambda)^{2}}{2} \mathbb{V} \mathbb{R}_{t}\left[\Delta \ln \left(c^{t+1}\right)\right] \\
& +\frac{(\lambda)^{2}}{2} \mathbb{V} \mathbb{A} \mathbb{R}_{t}\left[\Delta \ln \left(\bar{c}^{t+1}\right)\right]-(\gamma+\lambda) \lambda \operatorname{cov}_{t}\left[\Delta \ln \left(c^{t+1}\right), \Delta \ln \left(\bar{c}^{t+1}\right)\right]
\end{aligned}
$$

Com o surgimento de termos adicionais devidos à influência social podemos esperar que a presença da influência social possa impactar os "puzzles" econômicos.

Para uso na próxima seção vamos fazer mais algumas contas:

$$
\begin{aligned}
& \mathbb{V} \mathbb{R}_{t}\left[-(\gamma+\lambda) \Delta \ln \left(c^{t+1}\right)+\lambda \Delta \ln \left(\bar{c}^{t+1}\right)\right]= \\
& (\gamma+\lambda)^{2} \mathbb{V} \mathbb{R}_{t}\left[\Delta \ln \left(c^{t+1}\right)\right]+\lambda^{2} \mathbb{V} \mathbb{\mathbb { R } _ { t }}\left[\Delta \ln \left(\bar{c}^{t+1}\right)\right] \\
- & 2(\gamma+\lambda) \lambda \operatorname{cov}_{t}\left[\Delta \ln \left(c^{t+1}\right), \Delta \ln \left(\bar{c}^{t+1}\right)\right]
\end{aligned}
$$




\subsection{Considerações sobre os Puzzles}

Vamos conectar os desenvolvimentos das seções anteriores. Inicialmente vamos definir o log-retorno de um ativo por

$$
r^{i}=\ln R^{i}
$$

Agora, para o caso de um ativo sem risco que havíamos considerado temos:

$$
\begin{aligned}
r^{f} & =\ln R^{f} \\
& =-\ln \left(\mathbb{E}_{t}\left[M^{t+1}\right]\right) \\
& =-\ln \left(\mathbb{E}_{t}\left[\exp \left(-\left(\delta+\mu^{t+1}\right)\right)\right]\right) \\
& =-\ln \left(\mathbb{E}_{t}\left[\exp (-\delta) \exp \left(-\mu^{t+1}\right)\right]\right) \\
& =-\ln \left(\mathbb{E}_{t}\left[\beta_{a} \exp \left(-\mu^{t+1}\right)\right]\right) \\
& =-\ln \left(\beta_{a} \mathbb{E}_{t}\left[\exp \left(-\mu^{t+1}\right)\right]\right) \\
& =-\ln \beta_{a}-\ln \left(\mathbb{E}_{t}\left[\exp \left(-\mu^{t+1}\right)\right]\right)
\end{aligned}
$$

Esta notação é válida somente neste capítulo. Por outro lado, temos

$$
\begin{aligned}
\mu^{t+1} & >-\ln Y \Rightarrow \\
-\mu^{t+1} & <\ln Y \Rightarrow \\
\exp \left(-\mu^{t+1}\right) & <\exp (\ln Y) \Rightarrow \\
\mathbb{E}_{t}\left[\exp \left(-\mu^{t+1}\right)\right] & <\mathbb{E}_{t}[\exp (\ln Y)] \Rightarrow \\
-\mathbb{E}_{t}\left[\exp \left(-\mu^{t+1}\right)\right] & >-\mathbb{E}_{t}[Y]
\end{aligned}
$$

Assim, podemos concluir que 


$$
\begin{aligned}
r^{f}> & -\ln \left(\beta_{a}\right)-\mathbb{E}_{t}[Y] \\
> & -\ln \left(\beta_{a}\right)-\left(-(\gamma+\lambda) \mathbb{E}_{t}\left[\Delta \ln \left(c^{t+1}\right)\right]+\lambda \mathbb{E}_{t}\left[\Delta \ln \left(\bar{c}^{t+1}\right)\right]\right) \\
& -\left(\frac{(\gamma+\lambda)^{2}}{2} \mathbb{V} \mathbb{R}_{t}\left[\Delta \ln \left(c^{t+1}\right)\right]+\frac{\lambda^{2}}{2} \mathbb{V} \mathbb{A}_{t}\left[\Delta \ln \left(\bar{c}^{t+1}\right)\right]\right) \\
& -\left(-(\gamma+\lambda) \lambda \operatorname{cov}_{t}\left[\Delta \ln \left(c^{t+1}\right), \Delta \ln \left(\bar{c}^{t+1}\right)\right]\right) \\
> & -\ln \left(\beta_{a}\right)+(\gamma+\lambda) \mathbb{E}_{t}\left[\Delta \ln \left(c^{t+1}\right)\right]-\lambda \mathbb{E}_{t}\left[\Delta \ln \left(\bar{c}^{t+1}\right)\right] \\
& -\frac{(\gamma+\lambda)^{2}}{2} \mathbb{V A R}_{t}\left[\Delta \ln \left(c^{t+1}\right)\right]-\frac{\lambda^{2}}{2} \mathbb{V} \mathbb{\mathbb { R } _ { t }}\left[\Delta \ln \left(\bar{c}^{t+1}\right)\right] \\
& +(\gamma+\lambda) \lambda \operatorname{cov}_{t}\left[\Delta \ln \left(c^{t+1}\right), \Delta \ln \left(\bar{c}^{t+1}\right)\right]
\end{aligned}
$$

Esta desigualdade nos dá um limitante inferior para $r^{f}$, que possui vários termos a mais do que o tratamento clássico (sem influência social), isto mostra claramente que a presença da influência social pode impactar o "Riskfree Rate Puzzle"(RRP).

No modelo tradicional, sem influência social (note que fazendo $\lambda=0$ recuperamos a situação clássica), a equação fica:

$$
r^{f}=-\ln \beta_{a}+\gamma \mathbb{E}_{t}\left[\Delta \ln \left(c^{t+1}\right)\right]-\frac{\gamma^{2}}{2} \mathbb{V} \mathbb{R} \mathbb{R}_{t}\left[\Delta \ln \left(c^{t+1}\right)\right]
$$

De acordo com [bonomo][5], quando tentamos fazer uso dos dados (nem todos os países apresentam este problema) para obter $\beta$ obtemos $\ln \beta_{a}$ negativo, visto de outra forma, uma taxa de juros livre de risco baixa demais $\left(r_{f}\right)$. Este problema é chamado "Riskfree Rate Puzzle".

Ainda de acordo com [bonomo] [5] para países mais desenvolvidos, temos que o consumo agregado tem crescimento positivo $\left(\mathbb{E}_{t}\left[\Delta \ln \left(c^{t+1}\right)\right]>0\right)$ e a variância do consumo é pouco importante $\left(\mathbb{V} \mathbb{R}_{t}\left[\Delta \ln \left(c^{t+1}\right)\right]\right.$ pequeno). Enquanto no Brasil ocorre o oposto, onde verificou-se crescimento do consumo baixo e a volatilidade do consumo foi muito alta.

Porém quando olhamos as expectativas, os termos de variância do consumo 
esperado dos demais agentes $\left(\mathbb{V} \mathbb{R}_{t}\left[\Delta \ln \left(\bar{c}^{t+1}\right)\right]\right)$ e a covariância entre as duas variâncias de consumo $\left(\operatorname{cov}_{t}\left[\Delta \ln \left(c^{t+1}\right), \Delta \ln \left(\bar{c}^{t+1}\right)\right]\right)$ podem ser significativos, de modo que o limitante inferior que encontramos aumenta.

Nosso objetivo não é resolver o "puzzle", mas sim mostrar que ao considerar a hipótese de presença de influência social, temos outros elementos importantes a serem considerados no tratamento do "puzzle".

Vamos seguir adiante e fazer mais algumas considerações. Para o segue vamos supor que $\mu^{t+1} \approx-(\gamma+\lambda) \Delta \ln \left(c^{t+1}\right)+\lambda \Delta \ln \left(\bar{c}^{t+1}\right)$. Assim, para a variável $Y=\exp \left(-(\gamma+\lambda) \Delta \ln \left(c^{t+1}\right)+\lambda \Delta \ln \left(\bar{c}^{t+1}\right)\right)$ temos:

$$
\begin{aligned}
\frac{\sigma[Y]}{\mathbb{E}[Y]} & =\sqrt{\exp \left(\mathbb{V} \mathbb{R}_{t}\left[-(\gamma+\lambda) \Delta \ln \left(c^{t+1}\right)+\lambda \Delta \ln \left(\bar{c}^{t+1}\right)\right]\right)-1} \\
& \approx \sqrt{\mathbb{V} \mathbb{A} \mathbb{R}_{t}\left[-(\gamma+\lambda) \Delta \ln \left(c^{t+1}\right)+\lambda \Delta \ln \left(\bar{c}^{t+1}\right)\right]}
\end{aligned}
$$

Onde utilizamos os primeiros termos da série de Taylor da exponencial.

Seguindo a dedução clássica e aplicando o teorema de Hansen-Janganathan temos:

$$
\begin{aligned}
& \left(\frac{r_{M}-r_{f}}{\sigma_{M}}\right)^{2}= \\
& (\gamma+\lambda)^{2} \mathbb{V} \mathbb{A} \mathbb{R}_{t}\left[\Delta \ln \left(c^{t+1}\right)\right]+\lambda^{2} \mathbb{V} \mathbb{A} \mathbb{R}_{t}\left[\Delta \ln \left(\bar{c}^{t+1}\right)\right] \\
& -2(\gamma+\lambda) \lambda \operatorname{cov}_{t}\left[\Delta \ln \left(c^{t+1}\right), \Delta \ln \left(\bar{c}^{t+1}\right)\right]
\end{aligned}
$$

No modelo tradicional, sem influência social (note que fazendo $\lambda=0$ recuperamos a situação clássica), a equação fica:

$$
\frac{r_{M}-r_{f}}{\sigma_{M}}=\gamma \sqrt{\mathbb{V} \mathbb{A} \mathbb{R}_{t}\left[\Delta \ln \left(c^{t+1}\right)\right]}
$$

Como dito anteriormente, quando ajustamos com os dados, por exemplo do 
mercado americano, obtemos $\gamma$ alto demais. Note que a expressão que leva em a influência social possui novos termos de variância $\left(\mathbb{V} \mathbb{R} \mathbb{R}_{t}\left[\Delta \ln \left(\bar{c}^{t+1}\right)\right]\right)$ e covariância $\left(\operatorname{cov}_{t}\left[\Delta \ln \left(c^{t+1}\right), \Delta \ln \left(\bar{c}^{t+1}\right)\right]\right)$ que podem ser expressivos de modo a fazer com o termo $\gamma$, possa ser menor para "fechar as contas". Uma possibilidade é que as crenças do agente $a$ sejam tais que ele pense que seu consumo seja contrário ao que ele acredite que os demais agentes irão ter (de modo que teremos $\left.\operatorname{cov}_{t}\left[\Delta \ln \left(c^{t+1}\right), \Delta \ln \left(\bar{c}^{t+1}\right)\right]<0\right)$.

Note que as crenças são formadas pelas informações que chegam pelo canal de comunicação entre os investidores, de forma que mesmo sendo representativo, o agente $a$ pode ter crenças muito diferentes a respeito das ações dos demais em relação as suas próprias ações. Isso mostra que influência social pode, novamente, impactar outro "puzzle" econômico, no caso, o "Equity Premium Puzzle".

Finalmente, no próximo capítulo, fazemos um apanhado dos desenvolvimentos realizados e assinalamos algumas possibilidades de investigação futura. 


\section{Conclusão e Perspectivas}

I am now quick to reject any description of our work as demonstrating human irrationality. When the occasion arises, I carefully explain that research on heuristics and biases only refutes an unrealistic conception of rationality, which identifies it as comprehensive coherence... In my current view, the study of judgment biases requires attention to the interplay between intuitive and reflective thinking, which sometimes allows biased judgments and sometimes overrides or corrects them.

Daniel Kahneman

Autobiography, upon winning the Nobel Prize in Economic Sciences, 2002

Como mostrado nos capítulos anteriores, incluir a influência social (capturando o viés cognitivo da "inveja"), através de funções de utilidade generalizadas e com o uso de crenças heterogêneas de um agente sobre o comportamento dos demais, permitiu reconstruir aspectos de grande relevância da teoria de Finanças. Tais resultados vem a somar com percepção de Kahneman na citação acima de que o conceito tradicional de racionalidade econômica é incompleto e não realístico e que deve ser aprimorado.

Conseguimos obter um CAPM com influência social e estabelecer uma conexão entre aversão ao risco média efetiva do mercado, volatilidade e distribuição de riqueza dos agentes, estabelecendo a previsão empírica de que a volatilidade aumenta com a concentração da distribuição de riqueza dos agentes do mercado. Em seguida encontramos evidência empírica que suporta nossas conclusões. Continuando com a teoria, pudemos mostrar que influência social pode impactar situações onde a teoria tradicional de Finanças vem falhando há algumas décadas (com o EPP e o RRP).

A abordagem aqui apresentada de modelar a influência social por meio de sua inclusão em funções de utilidade poderia, em princípio, ser utilizada em Microeconomia de modo geral, onde certamente o fenômeno da influência social existe (exemplos de pessoas que alteram ou são influenciadas em seu consumo pelo de outras pessoas é algo bastante corriqueiro). Uma dificuldade neste caso é modelar como uma cesta de consumo de bens, onde podemos ter uma quantidade imensa de itens, poderia ser influenciada um a um pelas cestas de outras pessoas. Em nosso caso, o fator financeiro (riqueza) age como 
agregador que facilita a comparação entre os agentes. De qualquer modo, esta abordagem (com uso da influência social) permanece como um desafio para a Microeconomia.

Agora vamos falar um pouco sobre os próximos tópicos de pesquisa. A tese aqui apresentada tratou de um novo "framework" de trabalho, desenvolvendo alguns (porém de grande importância) aspectos de Finanças. Temos todo o resto da teoria a ser refeito levando em consideração a influência social.

Em particular o caso uniperiódico precisa ser completado, assim pretendemos desenvolver uma teoria de carteiras, bem como construir a fronteira eficiente e teoremas de separação associados. Devemos também analisar a situação mais geral em que os números de períodos de consumo é maior, para tratamento dos "puzzles".

Aplicar as ideias aqui apresentadas, em derivativos, mercado de seguros, Finanças Corporativas e em conjunto com outras ideias das Finanças Comportamentais parece ser bastante promissor. 


\section{Referências}

[1] M. Andersson, Social Influence in Stock Markets, Phd thesis in Psychology, University of Gothenburg, 2009

[2] A. Araújo, O Reconhecimento da Área de Finanças, Rev. Ciência Hoje, vol. 12 (68), nov/1990

[3] P. Bernstein, A História do Mercado de Capitais: o impacto da ciência e da tecnologia nos investimentos, Ed. Elsevier, 2008

[4] M. Billio, G. Jannin, B. Maillet, L. Pelizzon A New Generalized Utilitybased N-moments Measure of Performance, nov/2015

[5] M. Bonomo, Finanças, manuscrito de curso ministrado, disponível na internet, 2005

[6] E. Butkov, Mathematical Physics, Addison-Wesley, 1973, New York

[7] A. Cerny, Mathematical Techniques in Finance: Tools for Incomplete Markets, Princeton University Press, 2004

[8] N. Cetorelli, B. Hirtle, D. Morgan, S. Peristiani, J. Santos, Trends in Financial Market Concentration and Their Implications for Market Stability, FRBNY Economic Policy Review, mar/2007

[9] P. L. O. Costa Neto, O. R. Bekaman, Análise Estatística da Decisão, Ed. Edgard Blücher, 1980, São Paulo

[10] B. Dupire, A Few Myths in Quantitative Finance, Apresentação de seminário, disponível na internet, 2014

[11] M. Ferreira, S.B. Ramos, Mutual Fund Industry Competition and Concentration: International Evidence, SSRN Electronic Journal, jan/2009

[12] J.F.C. Guimarães, Existe Puzzle de Prêmio de Risco Acionário EPP) no Mercado Brasileiro? uma Análise do Período entre 1995 e 2013, Dissertação de Mestrado, FGV-RJ, 2014

[13] C. Huang, R.H. Litzenberger, Foundations for Financial Economics, Prentice Hall, 1993

[14] J.E. Ingersoll Jr., Theory of Financial Decision Making, RowmanLittlefield, 1987 
[15] D. Kahneman, Rápido e Devagar: duas formas de pensar, Ed. Objetiva, 2012, Rio de Janeiro

[16] D.M. Kreps, A Course in Microeconomic Theory, Princeton University Press, 1990

[17] S.X. Liang, K.C.J. Wei, Volatility and Stock Market Returns around the World, aug/2006

[18] A. Mas-Colell, M.D. Whinston, J.R. Green, Microeconomic Theory, Oxford University Press, 1995, New York

[19] H.N. Ozsoylev, Rational Expectations and Social Interaction in Financial Markets, 2006

[20] P. Papadopoulos, Revisiting Simple Concentration Indexes, OpenRisk White Paper, mar/2015

[21] J. Paudel, A Behavioral Approach to Stock Pricing, Mathematical Economics, 2010

[22] D. B. Pinho (coord.) Manual de Economia, Ed. Saraiva, 1988

[23] C. P. Samanez, R. C. Santos, Análise e Avaliação do Equity Premium Puzzle no Mercado Acionário Brasileiro sob Diferentes Contextos Econômicos, Rev. Bras. de Economia de Empresas, vol. 7 (2), 2007

[24] J. A. Scheinkman, U. Horst Equilibria in Systems of Social Interactions, J. of Economic Theory, 130, 2006

[25] J. A. Scheinkman, Social Interactions, The New Palgrave Dictionary of Economics, 2nd ed., Palgrave Macmillan, 2008

[26] P. P. S. Schirmer, C. J. Zimmer, Apreçamento de Ativos, manuscrito de curso ministrado, não publicado

[27] H. Shefrin, M. Statman, Behavioral Capital Asset Pricing Theory, J. of Financial and Quantitative Analysis, vol. 29 (3), sep/1994

[28] H. Shefrin, M. Statman, Behavioral Portfolio Theory, J. of Financial and Quantitative Analysis, vol. 35 (2), jun/2000

[29] H. Shefrin, A Behavioral Approach to Asset Pricing, Elsevier, 2008, 2nd ed. 
[30] R. H. Thaler, J. J. Siegel The Equity Premium Puzzle, J. of Economic Perspectives, vol. 11 (1), 1997, pp. 191-200

[31] C. Xu, Equity Risk Premium Puzzle and Investors' Behavioral Analysis Master Thesis, Columbia University, jan/2009 


\section{Índice Remissivo}

índice de Gini, 46, 68

caso uniperiódico, 29

coeficiente de aversão ao risco absoluto, 21

coeficiente de aversão ao risco relativo, 21

distribuição de Pareto, 46

dotação orçamentária, 82

estrutura probabilística das crenças, 30

estrutura probabilística dos retornos dos ativos, 30

fator de desconto estocástico, 83

fator subjetivo de desconto, 82

função de utilidade, 18

função de utilidade generalizada, 19

homo economicus, 13

influência social, 16

Lei dos Grandes Números, 45, 66

taxa marginal de substituição, 83

Utilidade Exponencial, 19

Utilidade Logarítmica, 26

utilidade marginal, 19

Utilidade Potência, 23

Utilidade Quadrática, 21

véu, 19

volatilidade do mercado, 42, 64 